\title{
APPLICATION OF STATISTICAL PREDICTIVE MODELS FOR FIELD FAILURE AND CISCO TESTING DATA (BIG DATA SOURCE: CISCO)
}

\author{
A Thesis \\ presented to \\ the Faculty of California Polytechnic State University, \\ San Luis Obispo
}

\author{
In Partial Fulfillment \\ of the Requirements for the Degree \\ Master of Science in Industrial Engineering
}

by

Alyssa Marie Carter

June 2017 
(C) 2017

Alyssa Marie Carter

ALL RIGHTS RESERVED 
COMMITTEE MEMBERSHIP

TITLE: Application of Statistical Predictive Models for Field Failure and Cisco

Testing Data

(Big Data Source: Cisco)

AUTHOR: $\quad$ Alyssa Marie Carter

DATE SUBMITTED: June 2017

COMMITTEE CHAIR: $\quad$ Reza Pouraghabagher, Ph.D.

Professor of Industrial Engineering

COMMITTEE MEMBER: $\quad$ Lizabeth Schlemer, Ph.D.

Professor of Industrial Engineering

COMMITTEE MEMBER: Xuan Wang, Ph.D.

Professor of Manufacturing Engineering 


\begin{abstract}
Application of Statistical Predictive Models for Field Failure and Cisco Testing Data (Big Data Source: Cisco)

Alyssa Marie Carter
\end{abstract}

Cisco was interested in how field failure categories relate to manufacturing test failures to better predict the tests that should be performed on a product after a return by a customer and what tests will be failed by a product before and after a return by a customer based on what type of failure occurred in the field. A study was conducted on one product type and 5 years of field data and the associated test data were captured. For each combination of field failure, shipping status, test area result, and test area a statistical model of the population proportion was created. The $95 \%$ and $99 \%$ confidence intervals were found. The critical test areas and a ranking of the criticality of the test areas were arranged from the confidence intervals. These findings will reduce the time spent on unnecessary tests. 


\section{ACKNOWLEDGMENTS}

I would like to thank Paul Ringelstein of Cisco for getting me started on this study and finding contacts for me after he had to change assignments. I would like to thank Senan Khairie and Jateen Pradhan of Cisco for providing me with data and guidance on what may be useful information from that data. I would especially like to thank Dr. Reza Pouraghabagher for helping me find an interesting topic, being a sounding board for statistical and thesis questions, and supporting me when I did not believe that I could finish my thesis on time to graduate. Finally, I would like to thank my family for supporting me and encouraging me to keep going in my education and not give up when I hit a wall. 


\section{TABLE OF CONTENTS}

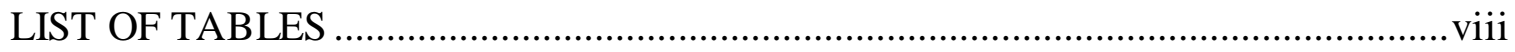

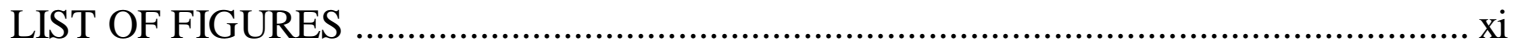

\section{CHAPTER}

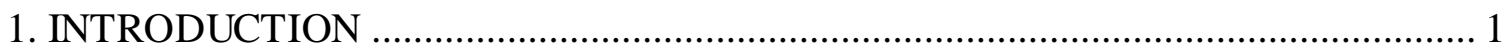

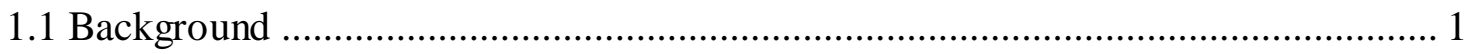

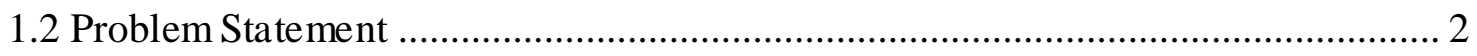

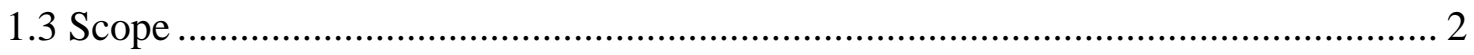

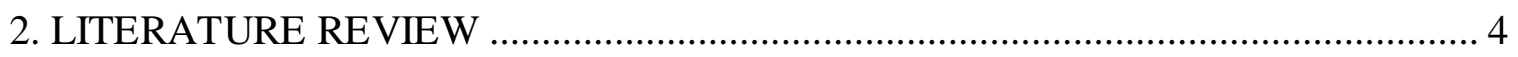

2.1 Big Data in Supply Chain ........................................................................... 4

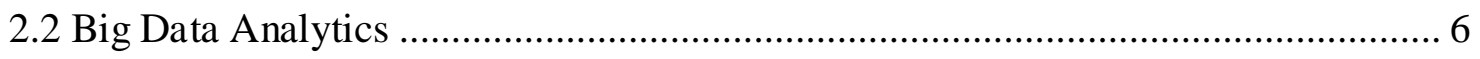

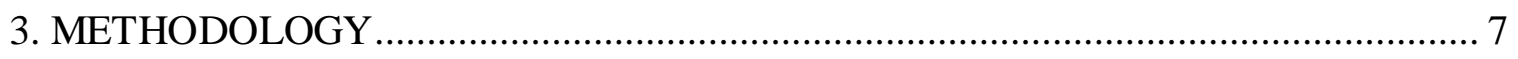

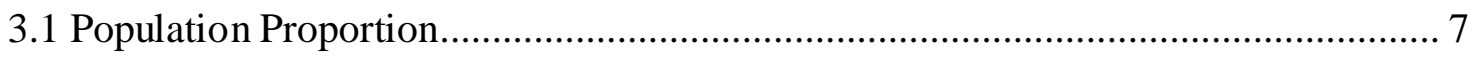

3.1.1 Filtering/Capturing Subsections ....................................................... 7

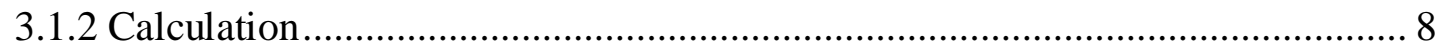

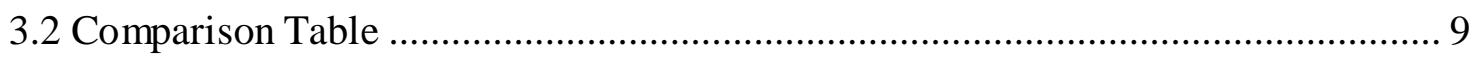

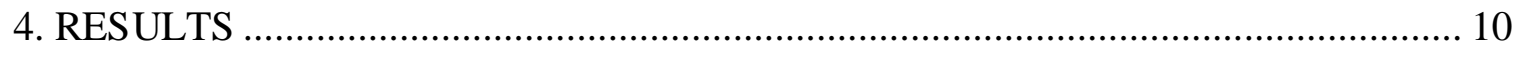

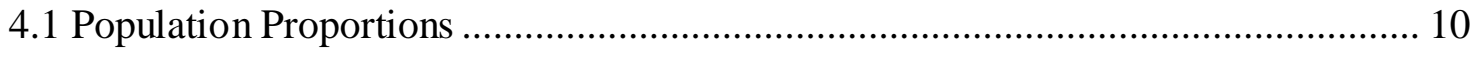

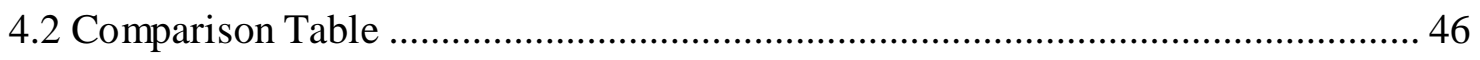

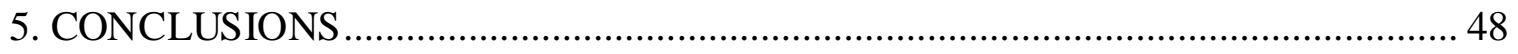

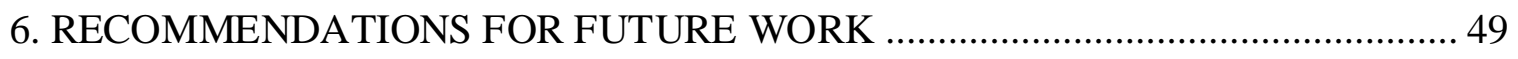


WORKS CITED 


\section{LIST OF TABLES}

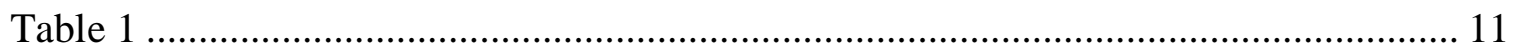

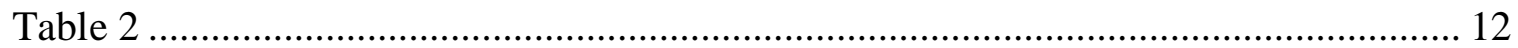

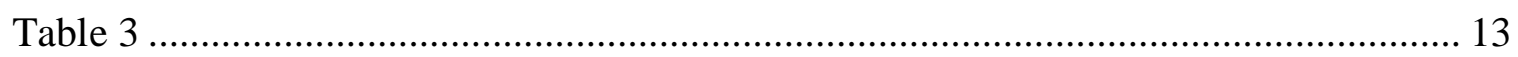

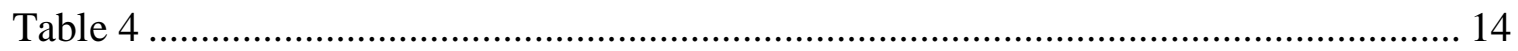

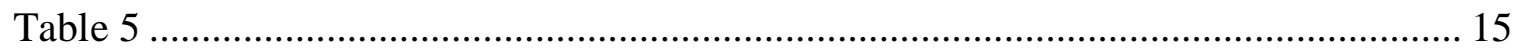

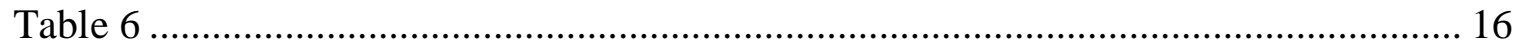

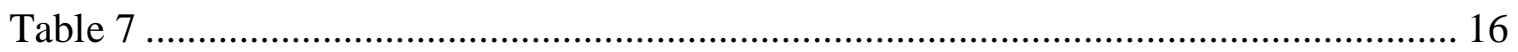

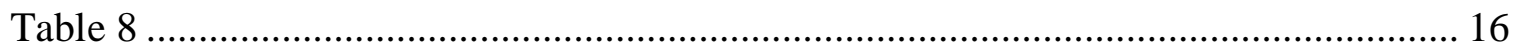

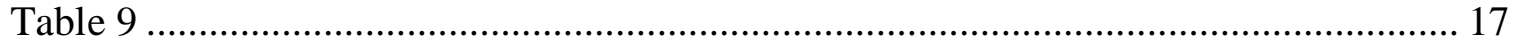

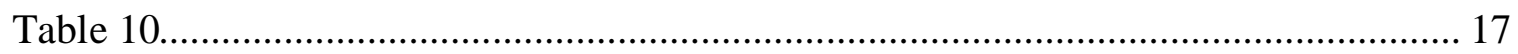

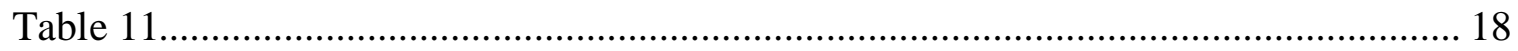

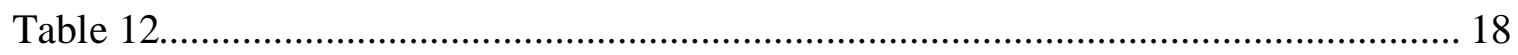

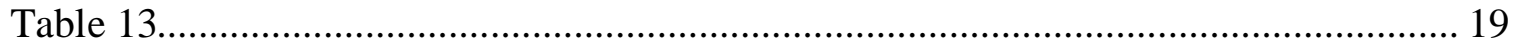

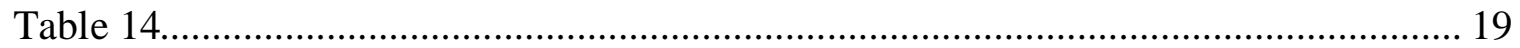

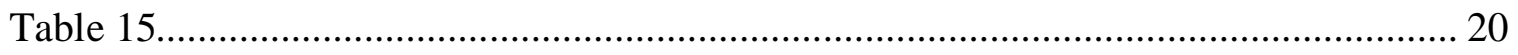

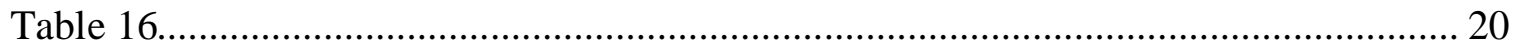

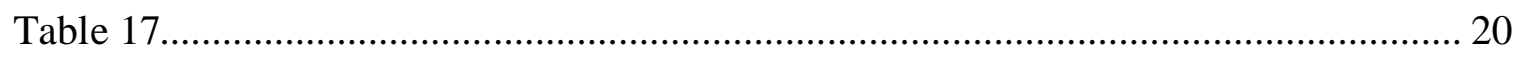

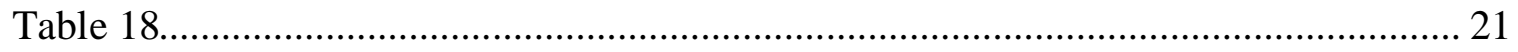

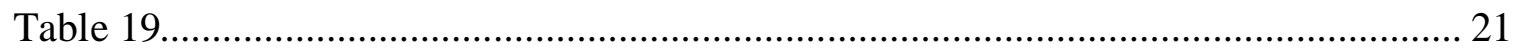

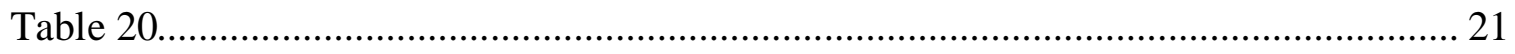

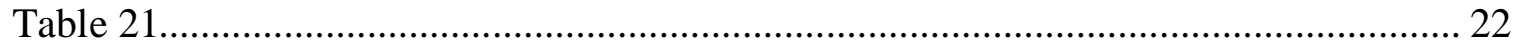




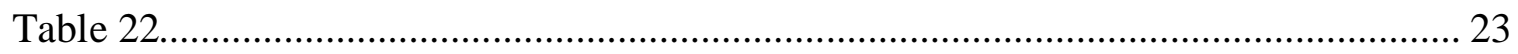

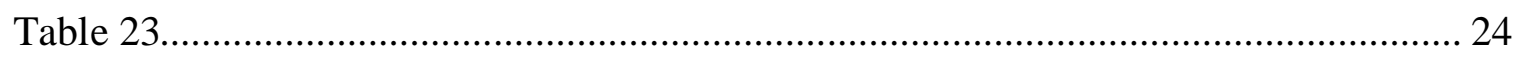

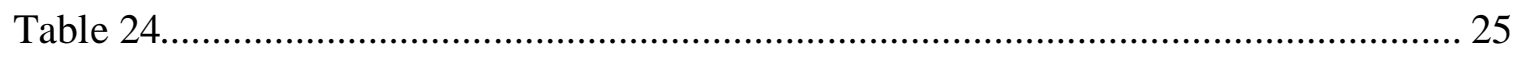

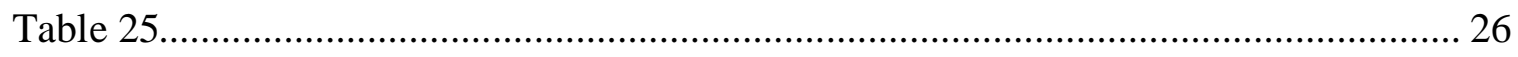

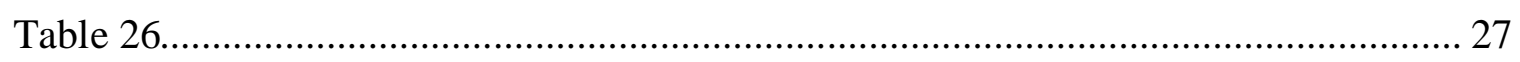

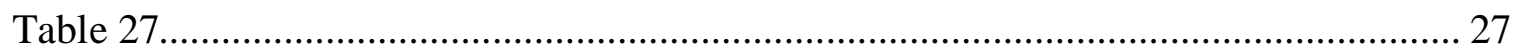

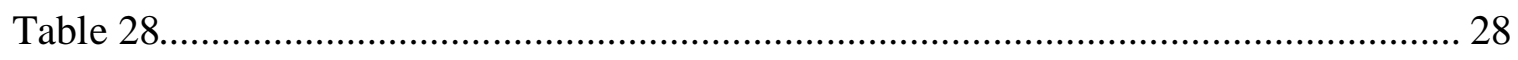

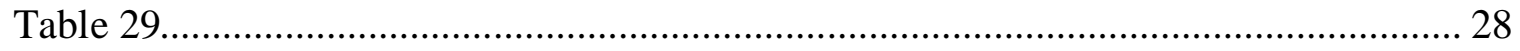

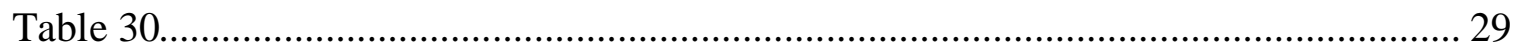

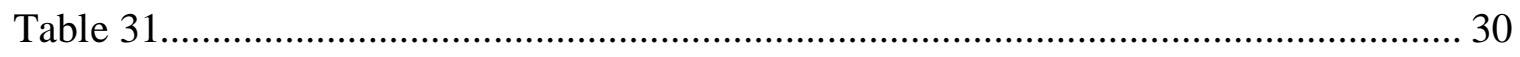

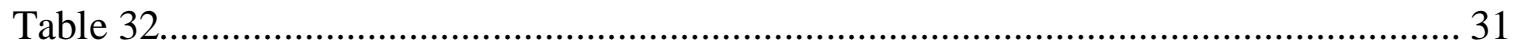

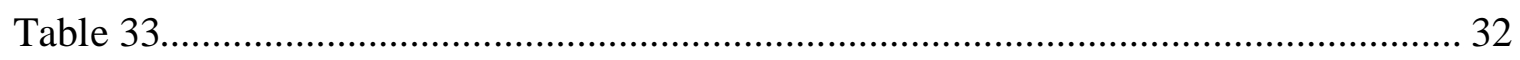

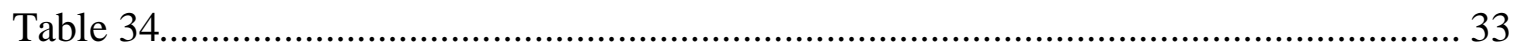

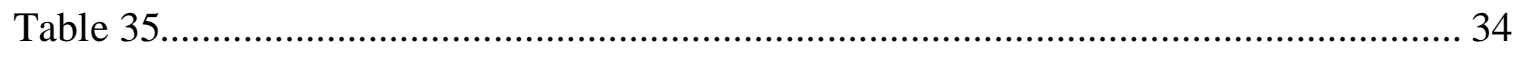

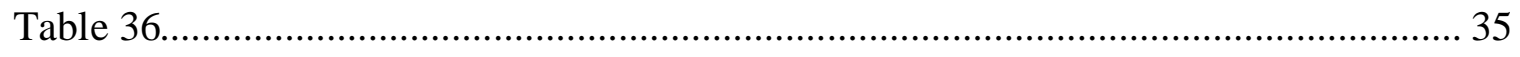

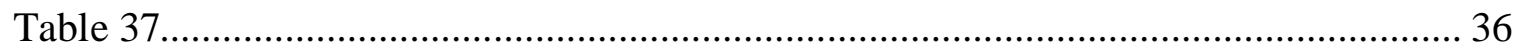

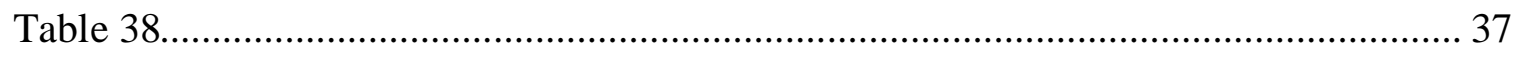

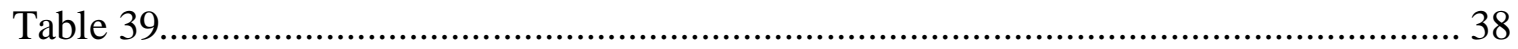

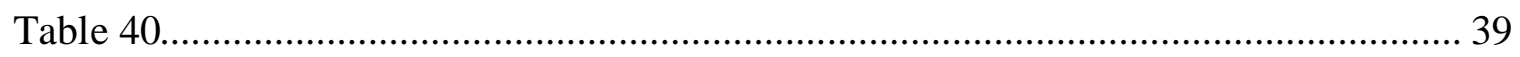

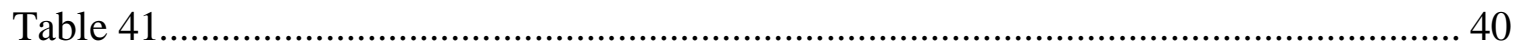

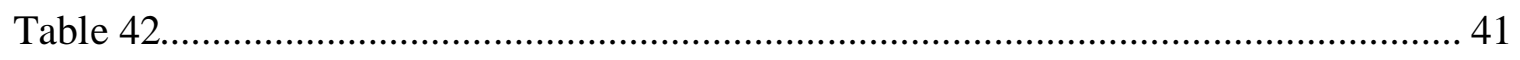

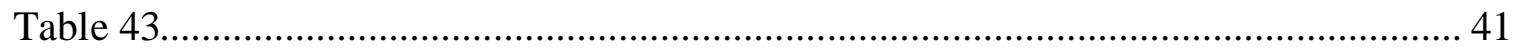

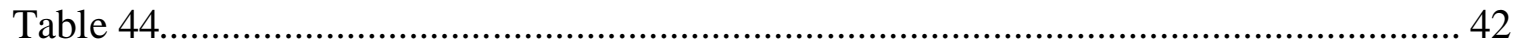




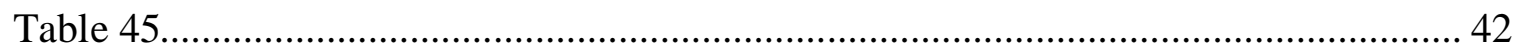

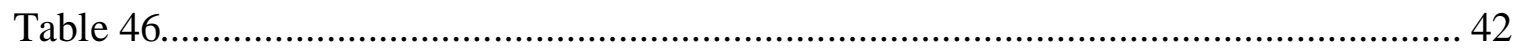

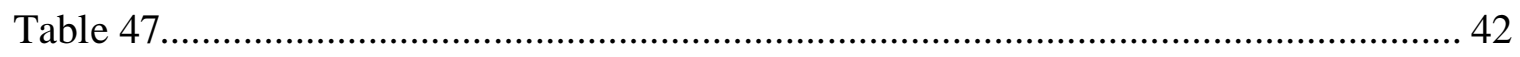

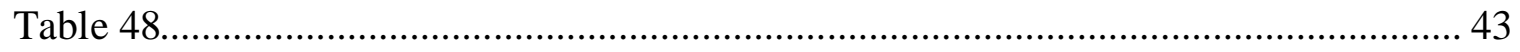

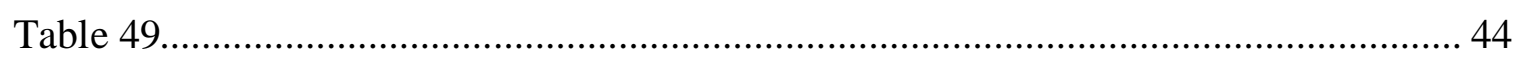

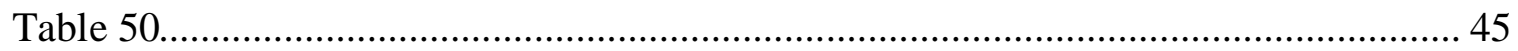

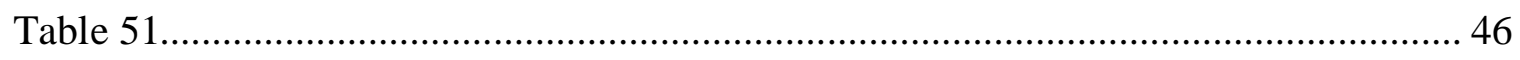

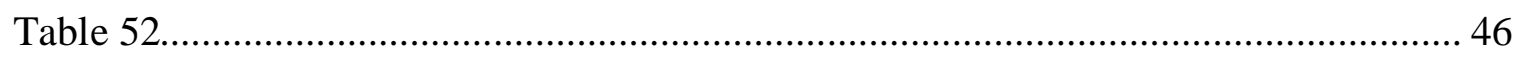

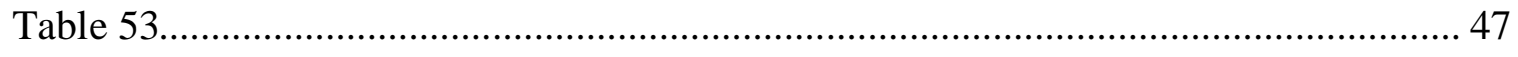




\section{LIST OF FIGURES}

Page

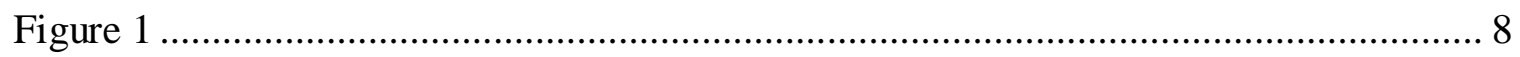

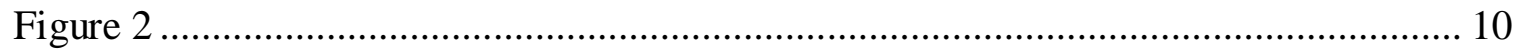

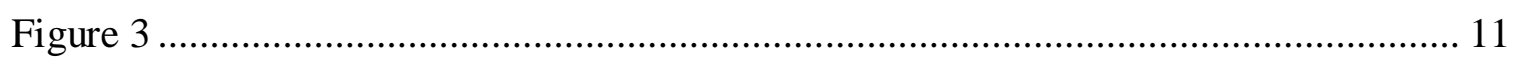




\section{INTRODUCTION}

Companies collect data every day and if they store that data it will accumulate and become more numerous than can be easily counted. These data sets are often referred to as big data. Storing big data is useful as it grants the ability to reference past occurrences or items. More than storing, though, there is a need for the organization and analysis of big data.

\subsection{Background}

Every product produced by Cisco goes through a series of tests and the results of those tests are recorded and stored in a database called the Cisco manufacturing report center (CMRC).

When a customer finds a product that has failed in the field, they work with a team at Cisco called the technical assistance center team (TAC). TAC helps the customer to define the failure of the product so that when it is sent back to Cisco for repair the testers have an idea of what to start testing. All of the details of the customer, their product, and the failure are combined into a service order that is processed and then stored in the return material authorization (RMA) database.

Tests performed on the returned product are stored in the CMRC with the data from before the product was shipped to the customer. The two databases, CMRC and RMA, 
store the data collected, but organization and analysis is necessary on the big data to combine the data sets and find useful information from the combination.

\subsection{Problem Statement}

Data is stored in the RMA and CMRC for field failures and manufacturing test failures, before and after shipping to a customer, respectively. Cisco wants to know how field failure categories relate to manufacturing test failures to better predict the tests that should be performed on a product after a return by a customer and what tests will be failed by a product before and after a return by a customer based on what type of failure occurred in the field.

\subsection{Scope}

This study is conducted on a single product type, UCSB-B200-M3, a Cisco blade server. For the product 17,950 rows and 24 columns of RMA data from 2012 to 2017 are extracted for analysis. The corresponding 346,634 rows and 34 columns of CMRC data are compiled from the unique serial numbers. There are 83 test areas, 2957 unique test failures, and 34 unique field failures in the data set. For this study only the top 10 field failures are considered. The objective of the study is to find the proportion of products that are failed, repaired, or passed in each test area before and after shipping to a customer with respect to the specific field failures. For example, the proportion of products that fail in XRAY that were returned to Cisco with motherboard failure. 
Along with the proportion analysis, an organization of the serial numbers and their corresponding field failures and number and list of test failures is done. 


\section{LITERATURE REVIEW}

Companies like Cisco accumulate data from many sources, from within the company and from external resources such as suppliers and customers. Through the collection and storage of this data big data databases are created. The organization and analysis of the data contained in these databases can be done in an infinite number of ways. It is important to companies to make use of the organization and analysis of their big data to do things like find trends in failed tests or estimate the percentage of products that will have specific issues.

It is the goal of this literature review to investigate the role of big data in supply chain and manufacturing, big data analytics, and methods of organization of big data. Though the data from is from a big data source, please note that this study looks at a large data set rather than big data.

\subsection{Big Data in Supply Chain}

Every step of the supply chain in a manufacturing setting creates data that is collected and stored. The big data from supply chain can be accessed and managed in ways that can provide important information for companies about key metrics that affect production and quality. 
"Big Data Driven Supply Chain Management and Business Administration" by Wang and Alexander (2015) looks at how big data has the potential to revolutionize supply chain dynamics and business management because of the value of integrated data sources and actionable information. They found that big data can help with efficiency, defect identification, procurement, supply chain collaboration, end-to-end supply chain execution, supply chain planning, inventory control and risk management, etc. The challenges of big data in supply chain management include but are not limited to data capture, data visualization, data integration, and data sharing.

The authors of "Manufacturing in the world: where next?" (Brennan, et al. 2015) discuss the changing of the configuration of manufacturing production around the globe. They address the major emerging themes that might impact global manufacturing including big data. They conclude that big data can have a great impact on global manufacturing, but it is just starting to change the landscape of global manufacturing and has not yet made a disruptive impact on it.

The impact of big data in manufacturing comes from the access to past data, the ability to see data in real time, and the analysis of data. Big data in manufacturing supply chain, like product testing results in quality or consumer demographics, is altering business practices in allowing the visibility, sharing, and analysis of information critical to processes. 


\subsection{Big Data Analytics}

Big data analytics is the process of examining big data to find patterns, trends, and other useful information. The inspection of big data can be done through advanced statistical analysis.

In "Big Data Analytics" (Russom 2011), the author talks about the importance of enterprises using advanced analytics to study big data to understand the current state of businesses. He notes the combination of two technical entities, big data and advanced analytics, and how it is an emerging practice in business intelligence. He recommends combining big data and advanced analytics to capture information and learn from big data.

Biswas and Sen propose to exploit the current state of data management, analytics, and visualization in "A Proposed Architecture for Big Data Driven Supply Chain Analytics" (2016). They describe the relevance of big data in supply chain strategy and the significance of big data analytics in supply chain management. 


\section{METHODOLOGY}

\subsection{Population Proportion}

The statistical tool of population proportion was used to analyze manufacturing test areas relation to field failure. Population proportion analysis takes the proportion of a sample from a population, in this case the population of the product type UCSB-B200-M3, and produces confidence intervals. The top 10 field failures were chosen for analysis.

\subsubsection{Filtering/Capturing Subsections}

The combination of RMA and CMRC data sets allowed for the division of the data by field failure and by test area. Serial numbers were separated into sections by field failure from the RMA data set and the corresponding serial numbers of the top 10 field failures were included in the CMRC data set through filtering by the unique serial numbers. From the subsection of data created through filtering, the unique combinations of serial number, shipping status, test area, and test area result were taken. The shipping status being test performed before or after shipping to customer and the test area results being failed, repaired, or passed. The resulting data set was then filtered by shipping status, test area result, and test area. A count of records was taken and recorded to be used to calculate the sample proportion. This was done for all combinations of the top 10 field failures and all shipping statuses, test areas, and test area results. For example, first finding the serial numbers that had a DIMM issue. Then, filtering the CMRC data set by those serial 
numbers. Next, taking the unique combinations of serial number, shipping status, test area, and test area result. Then, filtering the data by before shipping then by passed and finally by ASSY and taking a count of the records.

\subsubsection{Calculation}

The calculations of the $95 \%$ and $99 \%$ confidence intervals for the population proportions of each combination of the top 10 field failures and all test areas and test area failures were calculated in the statistical analysis software, Minitab. The equation that is used to calculate the confidence interval of a population proportion is in Figure 1.

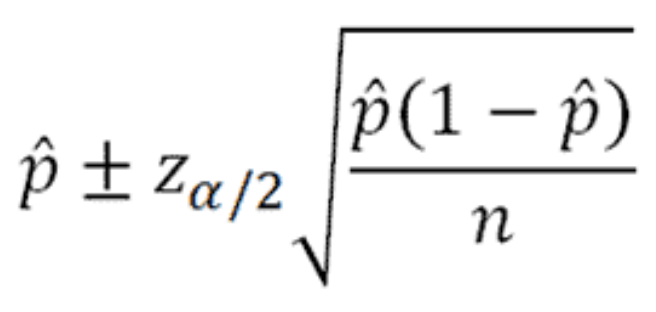

Figure 1

Where $\hat{p}=$ the population proportion estimate, $\mathrm{n}=$ the sample size, and $z_{\alpha / 2}=$ the appropriate confidence level from a $\mathrm{z}$ table. An example calculation from the study is the $95 \%$ confidence interval of the population proportion of the product with shipping status: before shipping, field failure: motherboard issue, test area: ICT, and test area code: failed. The calculation is as follows: $\hat{p}=0.036, \mathrm{n}=4497$, and $z_{\alpha / 2}=z_{0.025}=1.96$ so the $95 \%$ confidence interval is $(0.031,0.042)$ or $3.1 \%$ to $4.2 \%$. 


\subsection{Comparison Table}

Another thing taken from the crossing of the RMA and CMRC data sets was a comparison table of each unique serial number and the test failures from before and after shipping. It took the serial number, field failure, and return date of the product from the RMA data set and combined it with the associated test failures, sorting by before and after shipping. 


\section{RESULTS}

\subsection{Population Proportions}

Once a confidence interval is calculated how do you interpret it? If you have a $99 \%$ confidence interval for population proportion, it does not mean that there is a $99 \%$ probability that the population proportion will fall within the computed limits. Rather, it means that if the procedure of taking a sample and calculating the confidence interval was repeated many times the $99 \%$ of the times the population proportion will be included in the computed confidence intervals. This concept is visually interpreted in Figure 2.

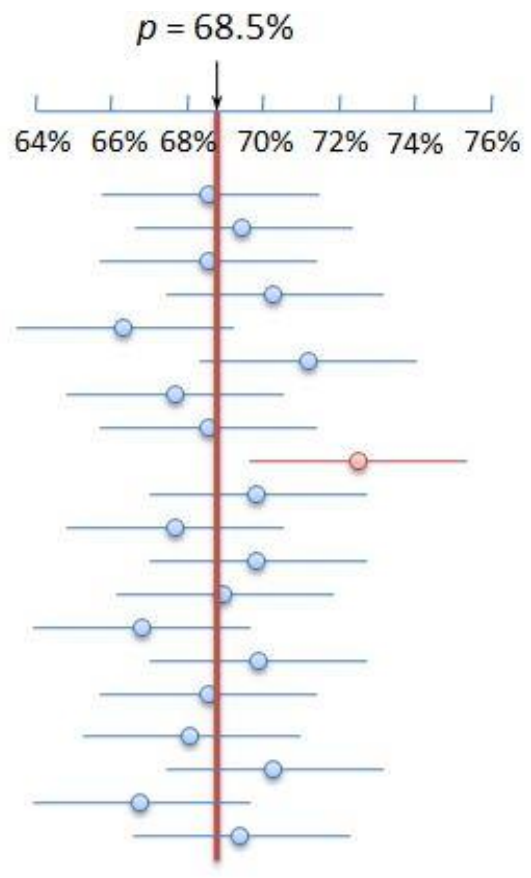

Figure 2

This study calculated $81095 \%$ confidence intervals and $81099 \%$ confidence intervals that are shown in Tables 1-30 and an explanation of acronyms is in Figure 3. 


\begin{tabular}{|l|l|}
\hline $\mathrm{Cl}$ & Confidence Interval \\
\hline $\mathrm{LCL}$ & Lower Confidence Limit \\
\hline $\mathrm{UCL}$ & Uper Confidence Limit \\
\hline
\end{tabular}

Figure 3

Table 1

\begin{tabular}{|c|c|c|c|c|c|c|c|c|}
\hline \multirow[t]{3}{*}{ Failed Before Shipping } & \multicolumn{4}{|c|}{ Field Failure } & \multicolumn{4}{|c|}{ Field Failure } \\
\hline & \multicolumn{4}{|c|}{ Motherboard Issue } & \multicolumn{4}{|c|}{ Motherboard Failure } \\
\hline & \multicolumn{2}{|c|}{$95 \% \mathrm{Cl}$} & \multicolumn{2}{|c|}{$99 \% \mathrm{Cl}$} & \multicolumn{2}{|c|}{$95 \% \mathrm{Cl}$} & \multicolumn{2}{|c|}{$99 \% \mathrm{Cl}$} \\
\hline Test Area & $\mathrm{LCL}$ & $\mathrm{UCL}$ & $\mathrm{LCL}$ & $\mathrm{UCL}$ & $\mathrm{LCL}$ & $\mathrm{UCL}$ & $\mathrm{LCL}$ & $\mathrm{UCL}$ \\
\hline DBGHTHV & $1.9539 \%$ & $2.8681 \%$ & $1.8335 \%$ & $3.0285 \%$ & $1.6919 \%$ & $2.7095 \%$ & $1.5634 \%$ & $2.8924 \%$ \\
\hline ICT & $3.0976 \%$ & $4.2130 \%$ & $2.9450 \%$ & $4.4044 \%$ & $2.2822 \%$ & $3.4363 \%$ & $2.1319 \%$ & $3.6400 \%$ \\
\hline \multicolumn{9}{|l|}{ OPCBPB } \\
\hline OSYSBI & $0.0138 \%$ & $0.1948 \%$ & $0.0075 \%$ & $0.2439 \%$ & & & & \\
\hline PCB2C & $4.4053 \%$ & $5.7050 \%$ & $4.2233 \%$ & $5.9247 \%$ & $3.7342 \%$ & $5.1574 \%$ & $3.5408 \%$ & $5.4023 \%$ \\
\hline PCBBI & $0.2035 \%$ & $0.5771 \%$ & $0.1684 \%$ & $0.6546 \%$ & $0.1234 \%$ & $0.5114 \%$ & $0.0939 \%$ & $0.5983 \%$ \\
\hline PCBCEV & & & & & $0.0008 \%$ & $0.1669 \%$ & $0.0002 \%$ & $0.2224 \%$ \\
\hline PCBDL & $0.3960 \%$ & $0.8724 \%$ & $0.3449 \%$ & $0.9655 \%$ & $0.2970 \%$ & $0.8144 \%$ & $0.2475 \%$ & $0.9208 \%$ \\
\hline PCBFA & $0.0626 \%$ & $0.3205 \%$ & $0.0453 \%$ & $0.3806 \%$ & $0.0185 \%$ & $0.2625 \%$ & $0.0101 \%$ & $0.3286 \%$ \\
\hline PCBFT & $7.0589 \%$ & $8.6513 \%$ & $6.8297 \%$ & $8.9156 \%$ & $6.3949 \%$ & $8.1850 \%$ & $6.1424 \%$ & $8.4856 \%$ \\
\hline PCBP2 & $0.0054 \%$ & $0.1606 \%$ & $0.0023 \%$ & $0.2061 \%$ & $0.0073 \%$ & $0.2163 \%$ & $0.0031 \%$ & $0.2776 \%$ \\
\hline PCBPEP & $0.0138 \%$ & $0.1948 \%$ & $0.0075 \%$ & $0.2439 \%$ & & & & \\
\hline PCBST & $4.2592 \%$ & $5.5400 \%$ & $4.0802 \%$ & $5.7568 \%$ & $4.2060 \%$ & $5.7035 \%$ & $4.0006 \%$ & $5.9597 \%$ \\
\hline POSTBI & $0.1380 \%$ & $0.4657 \%$ & $0.1100 \%$ & $0.5362 \%$ & $0.0844 \%$ & $0.4317 \%$ & $0.0611 \%$ & $0.5127 \%$ \\
\hline RSYSBI & $0.0242 \%$ & $0.2276 \%$ & $0.0150 \%$ & $0.2798 \%$ & & & & \\
\hline SYSADBG & $0.0006 \%$ & $0.1238 \%$ & $0.0001 \%$ & $0.1651 \%$ & & & & \\
\hline SYSAPK & $0.3601 \%$ & $0.8196 \%$ & $0.3116 \%$ & $0.9101 \%$ & $0.6074 \%$ & $1.2809 \%$ & $0.5333 \%$ & $1.4111 \%$ \\
\hline SYSBI & $0.0242 \%$ & $0.2276 \%$ & $0.0150 \%$ & $0.2798 \%$ & $0.0185 \%$ & $0.2625 \%$ & $0.0101 \%$ & $0.3286 \%$ \\
\hline SYSCEV & & & & & $0.0073 \%$ & $0.2163 \%$ & $0.0031 \%$ & $0.2776 \%$ \\
\hline SYSFA & $9.0806 \%$ & $10.8517 \%$ & $8.8226 \%$ & $11.1432 \%$ & $8.7539 \%$ & $10.7958 \%$ & $8.4606 \%$ & $11.1345 \%$ \\
\hline SYSFT & $5.1802 \%$ & $6.5745 \%$ & $4.9831 \%$ & $6.8085 \%$ & $4.5961 \%$ & $6.1516 \%$ & $4.3815 \%$ & $6.4166 \%$ \\
\hline SYSINT & $0.1540 \%$ & $0.4938 \%$ & $0.1242 \%$ & $0.5662 \%$ & $0.1036 \%$ & $0.4718 \%$ & $0.0771 \%$ & $0.5559 \%$ \\
\hline SYSPB & $0.0138 \%$ & $0.1948 \%$ & $0.0075 \%$ & $0.2439 \%$ & $0.0008 \%$ & $0.1669 \%$ & $0.0002 \%$ & $0.2224 \%$ \\
\hline SYSPEP & $0.0006 \%$ & $0.1238 \%$ & $0.0001 \%$ & $0.1651 \%$ & $0.0008 \%$ & $0.1669 \%$ & $0.0002 \%$ & $0.2224 \%$ \\
\hline SYSPM & $0.0490 \%$ & $0.2902 \%$ & $0.0342 \%$ & $0.3479 \%$ & $0.0487 \%$ & $0.3493 \%$ & $0.0323 \%$ & $0.4234 \%$ \\
\hline UNKNOWN & $0.0006 \%$ & $0.1238 \%$ & $0.0001 \%$ & $0.1651 \%$ & & & & \\
\hline \multicolumn{9}{|l|}{ WPCBASIC1 } \\
\hline XRAY & $0.2893 \%$ & $0.7129 \%$ & $0.2464 \%$ & $0.7980 \%$ & $0.2743 \%$ & $0.7775 \%$ & $0.2270 \%$ & $0.8817 \%$ \\
\hline XTD4C & & & & & $0.0008 \%$ & $0.1669 \%$ & $0.0002 \%$ & $0.2224 \%$ \\
\hline XTDSNT & $0.1540 \%$ & $0.4938 \%$ & $0.1242 \%$ & $0.5662 \%$ & $0.1438 \%$ & $0.5504 \%$ & $0.1115 \%$ & $0.6401 \%$ \\
\hline
\end{tabular}


Table 2

\begin{tabular}{|c|c|c|c|c|c|c|c|c|}
\hline \multirow{4}{*}{\begin{tabular}{|l|} 
Failed Before Shipping \\
\\
\\
Test Area \\
\end{tabular}} & \multicolumn{4}{|c|}{ Field Failure } & \multicolumn{4}{|c|}{ Field Failure } \\
\hline & \multicolumn{4}{|c|}{ DIMM Issue } & \multicolumn{4}{|c|}{ Blade Failure } \\
\hline & \multicolumn{2}{|c|}{$95 \% \mathrm{Cl}$} & \multicolumn{2}{|c|}{$99 \% \mathrm{Cl}$} & \multicolumn{2}{|c|}{$95 \% \mathrm{Cl}$} & \multicolumn{2}{|c|}{$99 \% \mathrm{Cl}$} \\
\hline & $\mathrm{LCL}$ & $\mathrm{UCL}$ & $\mathrm{LCL}$ & $\mathrm{UCL}$ & $\mathrm{LCL}$ & $\mathrm{UCL}$ & $\mathrm{LCL}$ & $\mathrm{UCL}$ \\
\hline \begin{tabular}{|l|} 
DBGHTHV \\
\end{tabular} & $0.8856 \%$ & $1.7433 \%$ & $0.7879 \%$ & $1.9060 \%$ & $2.2036 \%$ & $3.9049 \%$ & $1.9987 \%$ & $4.2178 \%$ \\
\hline ICT & $2.1677 \%$ & $3.4017 \%$ & $2.0102 \%$ & $3.6219 \%$ & $2.8377 \%$ & $4.7210 \%$ & $2.6037 \%$ & $5.0613 \%$ \\
\hline OРCBPB & $0.0009 \%$ & $0.1952 \%$ & $0.0002 \%$ & $0.2602 \%$ & & & & \\
\hline OSYSBI & $0.0085 \%$ & $0.2531 \%$ & $0.0036 \%$ & $0.3248 \%$ & & & & \\
\hline PCB2C & $3.0528 \%$ & $4.4776 \%$ & $2.8647 \%$ & $4.7270 \%$ & $5.3413 \%$ & $7.7780 \%$ & $5.0175 \%$ & $8.2013 \%$ \\
\hline PCBBI & $0.0382 \%$ & $0.3587 \%$ & $0.0236 \%$ & $0.4409 \%$ & $0.2496 \%$ & $1.0323 \%$ & $0.1900 \%$ & $1.2073 \%$ \\
\hline \multicolumn{9}{|l|}{ PCBCEV } \\
\hline PCBDL & $0.1212 \%$ & $0.5520 \%$ & $0.0902 \%$ & $0.6502 \%$ & $1.1794 \%$ & $2.5130 \%$ & $1.0334 \%$ & $2.7704 \%$ \\
\hline PCBFA & $0.0009 \%$ & $0.1952 \%$ & $0.0002 \%$ & $0.2602 \%$ & $0.1335 \%$ & $0.7893 \%$ & $0.0932 \%$ & $0.9457 \%$ \\
\hline PCBFT & $3.9834 \%$ & $5.5781 \%$ & $3.7681 \%$ & $5.8533 \%$ & $6.1183 \%$ & $8.6937 \%$ & $5.7718 \%$ & $9.1377 \%$ \\
\hline PCBP2 & & & & & $0.0015 \%$ & $0.3370 \%$ & $0.0003 \%$ & $0.4492 \%$ \\
\hline \multicolumn{9}{|l|}{ PCBPEP } \\
\hline PCBST & $2.6715 \%$ & $4.0184 \%$ & $2.4959 \%$ & $4.2559 \%$ & $2.5722 \%$ & $4.3822 \%$ & $2.3499 \%$ & $4.7115 \%$ \\
\hline POSTBI & $0.0987 \%$ & $0.5050 \%$ & $0.0715 \%$ & $0.5997 \%$ & $0.1335 \%$ & $0.7893 \%$ & $0.0932 \%$ & $0.9457 \%$ \\
\hline RSYSBI & & & & & $0.0015 \%$ & $0.3370 \%$ & $0.0003 \%$ & $0.4492 \%$ \\
\hline SYSADBG & & & & & $0.0375 \%$ & $0.5301 \%$ & $0.0205 \%$ & $0.6633 \%$ \\
\hline SYSAPK & $0.1927 \%$ & $0.6891 \%$ & $0.1517 \%$ & $0.7971 \%$ & $0.1706 \%$ & $0.8716 \%$ & $0.1235 \%$ & $1.0346 \%$ \\
\hline SYSBI & $0.0382 \%$ & $0.3587 \%$ & $0.0236 \%$ & $0.4409 \%$ & $0.2496 \%$ & $1.0323 \%$ & $0.1900 \%$ & $1.2073 \%$ \\
\hline SYSCEV & $0.0009 \%$ & $0.1952 \%$ & $0.0002 \%$ & $0.2602 \%$ & & & & \\
\hline SYSFA & $7.7862 \%$ & $9.9009 \%$ & $7.4867 \%$ & $10.2547 \%$ & $8.9287 \%$ & $11.9286 \%$ & $8.5123 \%$ & $12.4353 \%$ \\
\hline SYSFT & $5.2178 \%$ & $7.0055 \%$ & $4.9713 \%$ & $7.3100 \%$ & $5.2861 \%$ & $7.7124 \%$ & $4.9639 \%$ & $8.1342 \%$ \\
\hline SYSINT & $0.0085 \%$ & $0.2531 \%$ & $0.0036 \%$ & $0.3248 \%$ & $0.2908 \%$ & $1.1111 \%$ & $0.2255 \%$ & $1.2916 \%$ \\
\hline SYSPB & $0.0382 \%$ & $0.3587 \%$ & $0.0236 \%$ & $0.4409 \%$ & $0.6009 \%$ & $1.6435 \%$ & $0.5009 \%$ & $1.8572 \%$ \\
\hline SYSPEP & $0.0009 \%$ & $0.1952 \%$ & $0.0002 \%$ & $0.2602 \%$ & & & & \\
\hline SYSPM & $0.0085 \%$ & $0.2531 \%$ & $0.0036 \%$ & $0.3248 \%$ & $0.0015 \%$ & $0.3370 \%$ & $0.0003 \%$ & $0.4492 \%$ \\
\hline UNKNOWN & & & & & $0.0015 \%$ & $0.3370 \%$ & $0.0003 \%$ & $0.4492 \%$ \\
\hline \multicolumn{9}{|l|}{ WPCBASIC1 } \\
\hline XRAY & $0.2176 \%$ & $0.7338 \%$ & $0.1735 \%$ & $0.8448 \%$ & $0.3310 \%$ & $1.1890 \%$ & $0.2622 \%$ & $1.3748 \%$ \\
\hline \multicolumn{9}{|l|}{ XTD4C } \\
\hline XTDSNT & $0.0772 \%$ & $0.4573 \%$ & $0.0539 \%$ & $0.5481 \%$ & $0.0375 \%$ & $0.5301 \%$ & $0.0205 \%$ & $0.6633 \%$ \\
\hline
\end{tabular}


Table 3

\begin{tabular}{|c|c|c|c|c|c|c|c|c|}
\hline \multirow{4}{*}{\begin{tabular}{|l|} 
Failed Before Shipping \\
\\
Test Area \\
\end{tabular}} & \multicolumn{4}{|c|}{ Field Failure } & \multicolumn{4}{|c|}{ Field Failure } \\
\hline & \multicolumn{4}{|c|}{ Server Issue } & \multicolumn{4}{|c|}{ Hardware Failure } \\
\hline & \multicolumn{2}{|c|}{$95 \% \mathrm{Cl}$} & \multicolumn{2}{|c|}{$99 \% \mathrm{Cl}$} & \multicolumn{2}{|c|}{$95 \% \mathrm{Cl}$} & \multicolumn{2}{|c|}{$99 \% \mathrm{Cl}$} \\
\hline & LCL & UCL & $\mathrm{LCL}$ & $\mathrm{UCL}$ & LCL & UCL & $\mathrm{LCL}$ & UCL \\
\hline DBGHTHV & $2.2641 \%$ & $4.8622 \%$ & $1.9797 \%$ & $5.3613 \%$ & $1.2010 \%$ & $3.6430 \%$ & $0.9787 \%$ & $4.1511 \%$ \\
\hline ICT & $2.5640 \%$ & $5.2850 \%$ & $2.2597 \%$ & $5.8023 \%$ & $2.1666 \%$ & $5.1583 \%$ & $1.8559 \%$ & $5.7462 \%$ \\
\hline \multicolumn{9}{|l|}{ OPCBPB } \\
\hline OSYSBI & & & & & $0.0040 \%$ & $0.8675 \%$ & $0.0008 \%$ & $1.1551 \%$ \\
\hline PCB2C & $4.9490 \%$ & $8.4443 \%$ & $4.5178 \%$ & $9.0746 \%$ & $3.1847 \%$ & $6.6243 \%$ & $2.8009 \%$ & $7.2770 \%$ \\
\hline PCBBI & $0.1321 \%$ & $1.2352 \%$ & $0.0815 \%$ & $1.5168 \%$ & $0.0040 \%$ & $0.8675 \%$ & $0.0008 \%$ & $1.1551 \%$ \\
\hline \multicolumn{9}{|l|}{ PCBCEV } \\
\hline PCBDL & $0.2670 \%$ & $1.5743 \%$ & $0.1865 \%$ & $1.8848 \%$ & $0.9725 \%$ & $3.2523 \%$ & $0.7760 \%$ & $3.7369 \%$ \\
\hline PCBFA & $0.0031 \%$ & $0.6727 \%$ & $0.0006 \%$ & $0.8960 \%$ & $0.0379 \%$ & $1.1243 \%$ & $0.0162 \%$ & $1.4408 \%$ \\
\hline PCBFT & $6.6657 \%$ & $10.5860 \%$ & $6.1639 \%$ & $11.2777 \%$ & $3.8384 \%$ & $7.5239 \%$ & $3.4142 \%$ & $8.2121 \%$ \\
\hline PCBP2 & $0.0031 \%$ & $0.6727 \%$ & $0.0006 \%$ & $0.8960 \%$ & & & & \\
\hline PCBPEP & $0.0031 \%$ & $0.6727 \%$ & $0.0006 \%$ & $0.8960 \%$ & & & & \\
\hline PCBST & $1.9681 \%$ & $4.4356 \%$ & $1.7047 \%$ & $4.9156 \%$ & $2.6706 \%$ & $5.8961 \%$ & $2.3220 \%$ & $6.5178 \%$ \\
\hline POSTBI & $0.1321 \%$ & $1.2352 \%$ & $0.0815 \%$ & $1.5168 \%$ & $0.0040 \%$ & $0.8675 \%$ & $0.0008 \%$ & $1.1551 \%$ \\
\hline \multicolumn{9}{|l|}{\begin{tabular}{|l|} 
RSYSBI \\
\end{tabular}} \\
\hline SYSADBG & $0.0031 \%$ & $0.6727 \%$ & $0.0006 \%$ & $0.8960 \%$ & $0.0040 \%$ & $0.8675 \%$ & $0.0008 \%$ & $1.1551 \%$ \\
\hline SYSAPK & $0.3414 \%$ & $1.7382 \%$ & $0.2472 \%$ & $2.0616 \%$ & $0.0040 \%$ & $0.8675 \%$ & $0.0008 \%$ & $1.1551 \%$ \\
\hline \multicolumn{9}{|l|}{ SYSBI } \\
\hline \multicolumn{9}{|l|}{ SYSCEV } \\
\hline SYSFA & $8.7428 \%$ & $12.0915 \%$ & $8.1689 \%$ & $13.8445 \%$ & $8.0658 \%$ & $12.9328 \%$ & $7.4436 \%$ & $13.7891 \%$ \\
\hline SYSFT & $5.0552 \%$ & $8.5792 \%$ & $4.6193 \%$ & $9.2137 \%$ & $4.3688 \%$ & $8.2365 \%$ & $3.9145 \%$ & $8.9509 \%$ \\
\hline SYSINT & $0.1968 \%$ & $1.4069 \%$ & $0.1307 \%$ & $1.7036 \%$ & $0.0379 \%$ & $1.1243 \%$ & $0.0162 \%$ & $1.4408 \%$ \\
\hline SYSPB & $0.0293 \%$ & $0.8719 \%$ & $0.0125 \%$ & $1.1178 \%$ & $0.0040 \%$ & $0.8675 \%$ & $0.0008 \%$ & $1.1551 \%$ \\
\hline \multicolumn{9}{|l|}{ SYSPEP } \\
\hline SYSPM & $0.0293 \%$ & $0.8719 \%$ & $0.0125 \%$ & $1.1178 \%$ & $0.0040 \%$ & $0.8675 \%$ & $0.0008 \%$ & $1.1551 \%$ \\
\hline \multicolumn{9}{|l|}{ UNKNOWN } \\
\hline \multicolumn{9}{|l|}{ WPCBASIC1 } \\
\hline XRAY & $0.1321 \%$ & $1.2352 \%$ & $0.0815 \%$ & $1.5168 \%$ & $0.0379 \%$ & $1.1243 \%$ & $0.0162 \%$ & $1.4408 \%$ \\
\hline \multicolumn{9}{|l|}{ XTD4C } \\
\hline XTDSNT & $0.1321 \%$ & $1.2352 \%$ & $0.0815 \%$ & $1.5168 \%$ & $0.0040 \%$ & $0.8675 \%$ & $0.0008 \%$ & $1.1551 \%$ \\
\hline
\end{tabular}


Table 4

\begin{tabular}{|c|c|c|c|c|c|c|c|c|}
\hline \multirow{4}{*}{$\begin{array}{l}\text { Failed Before Shipping } \\
\text { Test Area }\end{array}$} & \multicolumn{4}{|c|}{ Field Failure } & \multicolumn{4}{|c|}{ Field Failure } \\
\hline & \multicolumn{4}{|c|}{ Memory Issue } & \multicolumn{4}{|c|}{ CPU Failure } \\
\hline & \multicolumn{2}{|c|}{$95 \% \mathrm{Cl}$} & \multicolumn{2}{|c|}{$99 \% \mathrm{Cl}$} & \multicolumn{2}{|c|}{$95 \% \mathrm{Cl}$} & \multicolumn{2}{|c|}{$99 \% \mathrm{Cl}$} \\
\hline & $\mathrm{LCL}$ & $\mathrm{UCL}$ & $\mathrm{LCL}$ & $\mathrm{UCL}$ & $\mathrm{LCL}$ & UCL & $\mathrm{LCL}$ & $\mathrm{UCL}$ \\
\hline DBGHTHV & $0.8874 \%$ & $3.9916 \%$ & $0.6613 \%$ & $4.6875 \%$ & $1.0034 \%$ & $4.5048 \%$ & $0.7479 \%$ & $5.2877 \%$ \\
\hline ICT & $1.9711 \%$ & $5.9347 \%$ & $1.6075 \%$ & $6.7510 \%$ & $1.1962 \%$ & $4.8802 \%$ & $0.9118 \%$ & $5.6884 \%$ \\
\hline \multicolumn{9}{|l|}{ ОРСВРВ } \\
\hline OSYSBI & & & & & $4.0273 \%$ & $9.4687 \%$ & $3.4546 \%$ & $10.5200 \%$ \\
\hline PCB2C & $2.3568 \%$ & $6.5603 \%$ & $1.9540 \%$ & $7.4104 \%$ & $0.1792 \%$ & $2.5129 \%$ & $0.0979 \%$ & $3.1363 \%$ \\
\hline PCBBI & $0.0620 \%$ & $1.8354 \%$ & $0.0265 \%$ & $2.3499 \%$ & & & & \\
\hline \multicolumn{9}{|l|}{ PCBCEV } \\
\hline PCBDL & $0.2794 \%$ & $2.5985 \%$ & $0.1724 \%$ & $3.1858 \%$ & $0.1792 \%$ & $2.5129 \%$ & $0.0979 \%$ & $3.1363 \%$ \\
\hline PCBFA & $0.0065 \%$ & $1.4167 \%$ & $0.0013 \%$ & $1.8847 \%$ & & & & \\
\hline PCBFT & $3.7651 \%$ & $8.6959 \%$ & $3.2420 \%$ & $9.6475 \%$ & $3.7959 \%$ & $9.1283 \%$ & $3.2415 \%$ & $10.1646 \%$ \\
\hline \multicolumn{9}{|l|}{\begin{tabular}{|l|} 
PCBP2 \\
\end{tabular}} \\
\hline \multicolumn{9}{|l|}{ PCBPEP } \\
\hline PCBST & $3.7651 \%$ & $8.6959 \%$ & $3.2420 \%$ & $9.6475 \%$ & $1.8047 \%$ & $5.9798 \%$ & $1.4413 \%$ & $6.8563 \%$ \\
\hline POSTBI & $0.0065 \%$ & $1.4167 \%$ & $0.0013 \%$ & $1.8847 \%$ & $0.0073 \%$ & $1.5997 \%$ & $0.0014 \%$ & $2.1276 \%$ \\
\hline RSYSBI & $0.0620 \%$ & $1.8354 \%$ & $0.0265 \%$ & $2.3499 \%$ & & & & \\
\hline SYSADBG & $0.0065 \%$ & $1.4167 \%$ & $0.0013 \%$ & $1.8847 \%$ & & & & \\
\hline SYSAPK & & & & & $0.0073 \%$ & $1.5997 \%$ & $0.0014 \%$ & $2.1276 \%$ \\
\hline SYSBI & $0.0620 \%$ & $1.8354 \%$ & $0.0265 \%$ & $2.3499 \%$ & $0.0073 \%$ & $1.5997 \%$ & $0.0014 \%$ & $2.1276 \%$ \\
\hline \multicolumn{9}{|l|}{ SYSCEV } \\
\hline SYSFA & $5.8808 \%$ & $11.6486 \%$ & $5.2162 \%$ & $12.7155 \%$ & $6.6564 \%$ & $13.1333 \%$ & $5.9070 \%$ & $14.3249 \%$ \\
\hline SYSFT & $5.6652 \%$ & $11.3572 \%$ & $5.0135 \%$ & $12.4137 \%$ & $2.8879 \%$ & $7.7503 \%$ & $2.4118 \%$ & $8.7219 \%$ \\
\hline \multicolumn{9}{|l|}{ SYSINT } \\
\hline SYSPB & $0.1585 \%$ & $2.2258 \%$ & $0.0866 \%$ & $2.7790 \%$ & $0.0701 \%$ & $0.0724 \%$ & $0.0300 \%$ & $2.6523 \%$ \\
\hline \multicolumn{9}{|l|}{ SYSPEP } \\
\hline SYSPM & & & & & $0.0073 \%$ & $1.5997 \%$ & $0.0014 \%$ & $2.1276 \%$ \\
\hline \multicolumn{9}{|l|}{ UNKNOWN } \\
\hline WPCBASIC1 & $0.0620 \%$ & $1.8354 \%$ & $0.0265 \%$ & $2.3499 \%$ & & & & \\
\hline XRAY & $0.1585 \%$ & $2.2258 \%$ & $0.0866 \%$ & $2.7790 \%$ & $0.0701 \%$ & $0.0724 \%$ & $0.0300 \%$ & $2.6523 \%$ \\
\hline \multicolumn{9}{|l|}{ XTD4C } \\
\hline XTDSNT & & & & & $0.0073 \%$ & $1.5997 \%$ & $0.0014 \%$ & $2.1276 \%$ \\
\hline
\end{tabular}


Table 5

\begin{tabular}{|c|c|c|c|c|c|c|c|c|}
\hline \multirow{4}{*}{$\begin{array}{l}\text { Failed Before Shipping } \\
\text { Test Area }\end{array}$} & \multicolumn{4}{|c|}{ Field Failure } & \multicolumn{4}{|c|}{ Field Failure } \\
\hline & \multicolumn{4}{|c|}{ Booting Issue } & \multicolumn{4}{|c|}{ DOA } \\
\hline & \multicolumn{2}{|c|}{$95 \% \mathrm{Cl}$} & \multicolumn{2}{|c|}{$99 \% \mathrm{Cl}$} & \multicolumn{2}{|c|}{$95 \% \mathrm{Cl}$} & \multicolumn{2}{|c|}{$99 \% \mathrm{Cl}$} \\
\hline & $\mathrm{LCL}$ & $\mathrm{UCL}$ & $\mathrm{LCL}$ & $\mathrm{UCL}$ & $\mathrm{LCL}$ & $\mathrm{UCL}$ & $\mathrm{LCL}$ & $\mathrm{UCL}$ \\
\hline DBGHTHV & $0.9463 \%$ & $4.7639 \%$ & $0.6859 \%$ & $5.6338 \%$ & $2.2256 \%$ & $7.7668 \%$ & $1.7557 \%$ & $8.9330 \%$ \\
\hline ICT & $2.3350 \%$ & $7.3202 \%$ & $1.8867 \%$ & $8.3498 \%$ & $0.6551 \%$ & $4.6236 \%$ & $0.4355 \%$ & $5.5790 \%$ \\
\hline \multicolumn{9}{|l|}{ ОРСВРВ } \\
\hline \multicolumn{9}{|l|}{ OSYSBI } \\
\hline PCB2C & $2.3350 \%$ & $7.3202 \%$ & $1.8867 \%$ & $8.3498 \%$ & $1.6658 \%$ & $6.7503 \%$ & $1.2705 \%$ & $7.8552 \%$ \\
\hline PCBBI & & & & & $0.0102 \%$ & $2.2172 \%$ & $0.0020 \%$ & $2.9458 \%$ \\
\hline \multicolumn{9}{|l|}{ PCBCEV } \\
\hline PCBDL & $0.0085 \%$ & $1.8492 \%$ & $0.0017 \%$ & $2.4584 \%$ & & & & \\
\hline \multicolumn{9}{|l|}{ PCBFA } \\
\hline PCBFT & $4.3999 \%$ & $10.5359 \%$ & $3.7590 \%$ & $11.7216 \%$ & $8.9591 \%$ & $17.6556 \%$ & $7.9445 \%$ & $19.2325 \%$ \\
\hline \multicolumn{9}{|c|}{ 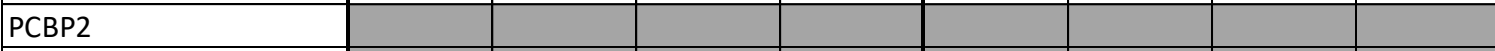 } \\
\hline \multicolumn{9}{|l|}{ PCBPEP } \\
\hline \begin{tabular}{|l|} 
PCBST \\
\end{tabular} & $2.8347 \%$ & $8.1394 \%$ & $2.3335 \%$ & $9.2126 \%$ & $0.6551 \%$ & $4.6236 \%$ & $0.4355 \%$ & $5.5790 \%$ \\
\hline POSTBI & $0.0811 \%$ & $2.3953 \%$ & $0.0347 \%$ & $3.0641 \%$ & $0.0102 \%$ & $2.2172 \%$ & $0.0020 \%$ & $2.9458 \%$ \\
\hline \multicolumn{9}{|l|}{ RSYSBI } \\
\hline \multicolumn{9}{|l|}{ SYSADBG } \\
\hline SYSAPK & $0.0811 \%$ & $2.3953 \%$ & $0.0347 \%$ & $3.0641 \%$ & & & & \\
\hline SYSBI & $0.0085 \%$ & $1.8492 \%$ & $0.0017 \%$ & $2.4584 \%$ & $0.0102 \%$ & $2.2172 \%$ & $0.0020 \%$ & $2.9458 \%$ \\
\hline \multicolumn{9}{|l|}{ SYSCEV } \\
\hline SYSFA & $7.7201 \%$ & $15.1496 \%$ & $6.8552 \%$ & $16.5065 \%$ & $9.3014 \%$ & $18.1060 \%$ & $8.2673 \%$ & $19.6962 \%$ \\
\hline SYSFT & $3.6065 \%$ & $9.3476 \%$ & $3.0324 \%$ & $10.4801 \%$ & $4.3403 \%$ & $11.1843 \%$ & $3.6516 \%$ & $12.5237 \%$ \\
\hline SYSINT & $0.0085 \%$ & $1.8492 \%$ & $0.0017 \%$ & $2.4584 \%$ & & & & \\
\hline SYSPB & $0.0085 \%$ & $1.8492 \%$ & $0.0017 \%$ & $2.4584 \%$ & & & & \\
\hline \multicolumn{9}{|l|}{ SYSPEP } \\
\hline \multicolumn{9}{|l|}{ SYSPM } \\
\hline \multicolumn{9}{|l|}{\begin{tabular}{|l|} 
UNKNOWN \\
\end{tabular}} \\
\hline WPCBASIC1 & & & & & $0.0102 \%$ & $2.2172 \%$ & $0.0020 \%$ & $2.9458 \%$ \\
\hline XRAY & $0.0811 \%$ & $2.3953 \%$ & $0.0347 \%$ & $3.0641 \%$ & $0.0974 \%$ & $2.8712 \%$ & $0.0416 \%$ & $3.6704 \%$ \\
\hline \multicolumn{9}{|l|}{ XTD4C } \\
\hline XTDSNT & $0.0085 \%$ & $1.8492 \%$ & $0.0017 \%$ & $2.4584 \%$ & & & & \\
\hline
\end{tabular}


Table 6

\begin{tabular}{|l|r|r|r|r|r|r|r|r|}
\hline Failed After Shipping & \multicolumn{3}{|c|}{ Field Failure } & \multicolumn{4}{c|}{ Field Failure } \\
\hline & \multicolumn{3}{|c|}{ Motherboard Issue } & \multicolumn{3}{c|}{ Motherboard Failure } \\
\hline & \multicolumn{2}{|c|}{$95 \% \mathrm{Cl}$} & \multicolumn{2}{|c|}{$99 \% \mathrm{Cl}$} & \multicolumn{2}{c|}{$95 \% \mathrm{Cl}$} & \multicolumn{2}{c|}{$99 \% \mathrm{Cl}$} \\
\hline Test Area & $\mathrm{LCL}$ & $\mathrm{UCL}$ & $\mathrm{LCL}$ & $\mathrm{UCL}$ & $\mathrm{LCL}$ & $\mathrm{UCL}$ & $\mathrm{LCL}$ & $\mathrm{UCL}$ \\
\hline FDBGHTHV & $9.4652 \%$ & $11.2672 \%$ & $9.2021 \%$ & $11.5633 \%$ & $8.4685 \%$ & $10.4825 \%$ & $8.1796 \%$ & $10.8170 \%$ \\
\hline FPCB2C & $5.3904 \%$ & $6.8088 \%$ & $5.1893 \%$ & $7.0465 \%$ & $4.1503 \%$ & $5.6394 \%$ & $3.9464 \%$ & $5.8943 \%$ \\
\hline FPCBAPK & $0.2204 \%$ & $0.6046 \%$ & $0.1836 \%$ & $0.6836 \%$ & $0.1036 \%$ & $0.4718 \%$ & $0.0771 \%$ & $0.5559 \%$ \\
\hline FPCBDG & $2.7480 \%$ & $3.8070 \%$ & $2.6045 \%$ & $3.9897 \%$ & $2.6623 \%$ & $3.8943 \%$ & $2.4996 \%$ & $4.1099 \%$ \\
\hline FPCBFT & $10.7070 \%$ & $12.6031 \%$ & $10.4287 \%$ & $12.9134 \%$ & $9.8126 \%$ & $11.9527 \%$ & $9.5032 \%$ & $12.3062 \%$ \\
\hline FPCBP2 & & & & & & & & \\
\hline FSYSFT & $0.1703 \%$ & $0.5218 \%$ & $0.1387 \%$ & $0.5959 \%$ & $0.0660 \%$ & $0.3909 \%$ & $0.0461 \%$ & $0.4686 \%$ \\
\hline FUNKNOWN & & & & & & & & \\
\hline
\end{tabular}

Table 7

\begin{tabular}{|l|r|r|r|r|r|r|r|r|}
\hline Failed After Shipping & \multicolumn{3}{|c|}{ Field Failure } & \multicolumn{4}{c|}{ Field Failure } \\
\hline & \multicolumn{3}{|c|}{ DIMM Issue } & \multicolumn{3}{c|}{ Blade Failure } \\
\hline & \multicolumn{2}{|c|}{$95 \% \mathrm{Cl}$} & \multicolumn{2}{|c|}{$99 \% \mathrm{Cl}$} & \multicolumn{2}{c|}{$95 \% \mathrm{Cl}$} & \multicolumn{2}{c|}{$99 \% \mathrm{Cl}$} \\
\hline Test Area & $\mathrm{LCL}$ & $\mathrm{UCL}$ & $\mathrm{LCL}$ & $\mathrm{UCL}$ & $\mathrm{LCL}$ & $\mathrm{UCL}$ & $\mathrm{LCL}$ & $\mathrm{UCL}$ \\
\hline FDBGHTHV & $3.7900 \%$ & $5.3514 \%$ & $3.5799 \%$ & $5.6215 \%$ & $8.0242 \%$ & $10.8985 \%$ & $7.6286 \%$ & $11.3868 \%$ \\
\hline FPCB2C & $3.5648 \%$ & $5.0862 \%$ & $3.3612 \%$ & $5.3503 \%$ & $5.2308 \%$ & $7.6468 \%$ & $4.9104 \%$ & $8.0670 \%$ \\
\hline FPCBAPK & $0.0569 \%$ & $0.4086 \%$ & $0.0378 \%$ & $0.4953 \%$ & $0.0984 \%$ & $0.7053 \%$ & $0.0653 \%$ & $0.8647 \%$ \\
\hline FPCBDG & $1.8561 \%$ & $3.0130 \%$ & $1.7109 \%$ & $3.2214 \%$ & $2.7845 \%$ & $4.6533 \%$ & $2.5527 \%$ & $4.9915 \%$ \\
\hline FPCBFT & $6.5301 \%$ & $8.4951 \%$ & $6.2549 \%$ & $8.8264 \%$ & $12.1808 \%$ & $15.5686 \%$ & $11.6997 \%$ & $16.1321 \%$ \\
\hline FPCBP2 & & & & & & & & \\
\hline FSYSFT & $0.0009 \%$ & $0.1952 \%$ & $0.0002 \%$ & $0.2602 \%$ & $0.0661 \%$ & $0.6192 \%$ & $0.0407 \%$ & $0.7608 \%$ \\
\hline FUNKNOWN & & & & & $0.0015 \%$ & $0.3370 \%$ & $0.0003 \%$ & $0.4492 \%$ \\
\hline
\end{tabular}

Table 8

\begin{tabular}{|l|r|r|r|r|r|r|r|r|}
\hline Failed After Shipping & \multicolumn{4}{|c|}{ Field Failure } & \multicolumn{3}{c|}{ Field Failure } \\
\hline & \multicolumn{3}{|c|}{ Server Issue } & \multicolumn{3}{c|}{ Hardware Failure } \\
\hline & \multicolumn{2}{|c|}{$95 \% \mathrm{Cl}$} & \multicolumn{2}{|c|}{$99 \% \mathrm{Cl}$} & \multicolumn{2}{c|}{$95 \% \mathrm{Cl}$} & \multicolumn{2}{c|}{$99 \% \mathrm{Cl}$} \\
\hline Test Area & $\mathrm{LCL}$ & $\mathrm{UCL}$ & $\mathrm{LCL}$ & $\mathrm{UCL}$ & $\mathrm{LCL}$ & $\mathrm{UCL}$ & $\mathrm{LCL}$ & $\mathrm{UCL}$ \\
\hline FDBGHTHV & $8.0831 \%$ & $12.3040 \%$ & $7.5308 \%$ & $12.0388 \%$ & $8.3456 \%$ & $13.2749 \%$ & $7.7129 \%$ & $14.1398 \%$ \\
\hline FPCB2C & $6.2333 \%$ & $10.0537 \%$ & $5.7482 \%$ & $10.7311 \%$ & $3.5756 \%$ & $7.1654 \%$ & $3.1672 \%$ & $7.8398 \%$ \\
\hline FPCBAPK & $0.1968 \%$ & $1.4069 \%$ & $0.1307 \%$ & $1.7036 \%$ & $0.0968 \%$ & $1.3637 \%$ & $0.0529 \%$ & $1.7046 \%$ \\
\hline FPCBDG & $2.5640 \%$ & $5.2850 \%$ & $2.2597 \%$ & $5.8023 \%$ & $2.2915 \%$ & $5.3438 \%$ & $1.9711 \%$ & $5.9404 \%$ \\
\hline FPCBFT & $12.4190 \%$ & $17.3755 \%$ & $11.7411 \%$ & $18.2139 \%$ & $12.3221 \%$ & $18.0054 \%$ & $11.5591 \%$ & $18.9743 \%$ \\
\hline FPCBP2 & & & & & $0.0040 \%$ & $0.8675 \%$ & $0.0008 \%$ & $1.1551 \%$ \\
\hline FSYSFT & $0.0750 \%$ & $1.0577 \%$ & $0.0409 \%$ & $1.3226 \%$ & $0.0379 \%$ & $1.1243 \%$ & $0.0162 \%$ & $1.4408 \%$ \\
\hline FUNKNOWN & & & & & & & & \\
\hline
\end{tabular}


Table 9

\begin{tabular}{|l|r|r|r|r|r|r|r|r|}
\hline Failed After Shipping & \multicolumn{4}{|c|}{ Field Failure } & \multicolumn{4}{c|}{ Field Failure } \\
\hline & \multicolumn{3}{|c|}{ Memory Issue } & \multicolumn{3}{c|}{ CPU Failure } \\
\hline & \multicolumn{2}{|c|}{$95 \% \mathrm{Cl}$} & \multicolumn{2}{|c|}{$99 \% \mathrm{Cl}$} & \multicolumn{2}{c|}{$95 \% \mathrm{Cl}$} & \multicolumn{2}{c|}{$99 \% \mathrm{Cl}$} \\
\hline Test Area & $\mathrm{LCL}$ & $\mathrm{UCL}$ & $\mathrm{LCL}$ & $\mathrm{UCL}$ & $\mathrm{LCL}$ & $\mathrm{UCL}$ & $\mathrm{LCL}$ & $\mathrm{UCL}$ \\
\hline FDBGHTHV & $6.0971 \%$ & $11.9393 \%$ & $5.4199 \%$ & $13.0163 \%$ & $5.4446 \%$ & $11.4836 \%$ & $4.7705 \%$ & $12.6163 \%$ \\
\hline FPCB2C & $3.3550 \%$ & $8.0930 \%$ & $2.8640 \%$ & $9.0177 \%$ & $4.9672 \%$ & $10.8167 \%$ & $4.3254 \%$ & $11.9237 \%$ \\
\hline FPCBAPK & $0.2794 \%$ & $2.5985 \%$ & $0.1724 \%$ & $3.1858 \%$ & & & & \\
\hline FPCBDG & $1.2331 \%$ & $4.6530 \%$ & $0.9571 \%$ & $5.3931 \%$ & $2.0155 \%$ & $6.3393 \%$ & $1.6280 \%$ & $7.2363 \%$ \\
\hline FPCBFT & $9.8681 \%$ & $16.7910 \%$ & $9.0028 \%$ & $18.0128 \%$ & $12.4635 \%$ & $20.4969 \%$ & $11.4376 \%$ & $21.8909 \%$ \\
\hline FPCBP2 & & & & & & & & \\
\hline FSYSFT & & & & & & & & \\
\hline FUNKNOWN & & & & & & & & \\
\hline
\end{tabular}

Table 10

\begin{tabular}{|l|r|r|r|r|r|r|r|r|}
\hline Failed After Shipping & \multicolumn{4}{|c|}{ Field Failure } & \multicolumn{4}{c|}{ Field Failure } \\
\hline & \multicolumn{3}{|c|}{ Booting Issue } & \multicolumn{3}{c|}{ DOA } \\
\hline & \multicolumn{2}{|c|}{$95 \% \mathrm{Cl}$} & \multicolumn{2}{c|}{$99 \% \mathrm{Cl}$} & \multicolumn{2}{c|}{$95 \% \mathrm{Cl}$} & \multicolumn{2}{c|}{$99 \% \mathrm{Cl}$} \\
\hline Test Area & $\mathrm{LCL}$ & $\mathrm{UCL}$ & $\mathrm{LCL}$ & $\mathrm{UCL}$ & $\mathrm{LCL}$ & $\mathrm{UCL}$ & $\mathrm{LCL}$ & $\mathrm{UCL}$ \\
\hline FDBGHTHV & $9.7323 \%$ & $17.7686 \%$ & $8.7593 \%$ & $19.2033 \%$ & $5.9458 \%$ & $13.5374 \%$ & $5.1278 \%$ & $14.9741 \%$ \\
\hline FPCB2C & $5.2105 \%$ & $11.7078 \%$ & $4.5078 \%$ & $12.9419 \%$ & $3.7171 \%$ & $10.2254 \%$ & $3.0856 \%$ & $11.5204 \%$ \\
\hline FPCBAPK & $0.3657 \%$ & $3.3897 \%$ & $0.2257 \%$ & $4.1519 \%$ & $0.0102 \%$ & $2.2172 \%$ & $0.0020 \%$ & $2.9458 \%$ \\
\hline FPCBDG & $2.5832 \%$ & $7.7313 \%$ & $2.1079 \%$ & $8.7832 \%$ & $1.3971 \%$ & $6.2322 \%$ & $1.0419 \%$ & $7.3037 \%$ \\
\hline FPCBFT & $11.4889 \%$ & $19.9825 \%$ & $10.4329 \%$ & $21.4745 \%$ & $10.6832 \%$ & $19.8954 \%$ & $9.5744 \%$ & $21.5355 \%$ \\
\hline FPCBP2 & & & & & & & & \\
\hline FSYSFT & & & & & $0.0974 \%$ & $2.8712 \%$ & $0.0416 \%$ & $3.6704 \%$ \\
\hline FUNKNOWN & & & & & & & & \\
\hline
\end{tabular}


Table 11

\begin{tabular}{|l|c|c|c|c|c|c|c|c|}
\hline Repaired Before Shipping & \multicolumn{3}{|c|}{ Field Failure } & \multicolumn{4}{c|}{ Field Failure } \\
\hline & \multicolumn{3}{|c|}{ Motherboard Issue } & \multicolumn{3}{c|}{ Motherboard Failure } \\
\hline & \multicolumn{2}{|c|}{$95 \% \mathrm{Cl}$} & \multicolumn{2}{|c|}{$99 \% \mathrm{Cl}$} & \multicolumn{2}{c|}{$95 \% \mathrm{Cl}$} & \multicolumn{2}{c|}{$99 \% \mathrm{Cl}$} \\
\hline Test Area & $\mathrm{LCL}$ & $\mathrm{UCL}$ & $\mathrm{LCL}$ & $\mathrm{UCL}$ & $\mathrm{LCL}$ & $\mathrm{UCL}$ & $\mathrm{LCL}$ & $\mathrm{UCL}$ \\
\hline DBGHTHV & $0.0490 \%$ & $0.2902 \%$ & $0.0342 \%$ & $0.3479 \%$ & $0.0844 \%$ & $0.4317 \%$ & $0.0611 \%$ & $0.5127 \%$ \\
\hline ICT & & & & & & & & \\
\hline IPAOI & $9.3156 \%$ & $11.1057 \%$ & $9.0544 \%$ & $11.4000 \%$ & $10.4724 \%$ & $12.6701 \%$ & $10.1536 \%$ & $13.0322 \%$ \\
\hline IPMVI & $3.2626 \%$ & $4.4035 \%$ & $3.1060 \%$ & $4.5987 \%$ & $3.1549 \%$ & $4.4793 \%$ & $2.9774 \%$ & $4.7091 \%$ \\
\hline PCB2C & & & & & & & & \\
\hline PCBBI & & & & & & & & \\
\hline PCBDG & $0.0768 \%$ & $0.3502 \%$ & $0.0572 \%$ & $0.4126 \%$ & $0.0327 \%$ & $0.3066 \%$ & $0.0202 \%$ & $0.3769 \%$ \\
\hline PCBFA & & & & & $0.0008 \%$ & $0.1669 \%$ & $0.0002 \%$ & $0.2224 \%$ \\
\hline PCBFT & $0.0006 \%$ & $0.1238 \%$ & $0.0001 \%$ & $0.1651 \%$ & & & & \\
\hline SYSBI & $0.0006 \%$ & $0.1238 \%$ & $0.0001 \%$ & $0.1651 \%$ & & & & \\
\hline SYSFA & $0.9420 \%$ & $1.6141 \%$ & $0.8600 \%$ & $1.7372 \%$ & $1.0882 \%$ & $1.9367 \%$ & $0.9866 \%$ & $2.0936 \%$ \\
\hline SYSFT & $0.2374 \%$ & $0.6319 \%$ & $0.1991 \%$ & $0.7125 \%$ & $0.1859 \%$ & $0.6273 \%$ & $0.1483 \%$ & $0.7222 \%$ \\
\hline SYSPB & $0.0006 \%$ & $0.1238 \%$ & $0.0001 \%$ & $0.1651 \%$ & $0.0008 \%$ & $0.1669 \%$ & $0.0002 \%$ & $0.2224 \%$ \\
\hline
\end{tabular}

Table 12

\begin{tabular}{|c|c|c|c|c|c|c|c|c|}
\hline \multirow[t]{3}{*}{ Repaired Before Shipping } & \multicolumn{4}{|c|}{ Field Failure } & \multicolumn{4}{|c|}{ Field Failure } \\
\hline & \multicolumn{4}{|c|}{ DIMM Issue } & \multicolumn{4}{|c|}{ Blade Failure } \\
\hline & \multicolumn{2}{|c|}{$95 \% \mathrm{Cl}$} & \multicolumn{2}{|c|}{$99 \% \mathrm{Cl}$} & \multicolumn{2}{|c|}{$95 \% \mathrm{Cl}$} & \multicolumn{2}{|c|}{$99 \% \mathrm{Cl}$} \\
\hline Test Area & LCL & UCL & $\mathrm{LCL}$ & $\mathrm{UCL}$ & $\mathrm{LCL}$ & $\mathrm{UCL}$ & $\mathrm{LCL}$ & UCL \\
\hline DBGHTHV & $0.0772 \%$ & $0.4573 \%$ & $0.0539 \%$ & $0.5481 \%$ & $0.0375 \%$ & $0.5301 \%$ & $0.0205 \%$ & $0.6633 \%$ \\
\hline \multicolumn{9}{|l|}{ ICT } \\
\hline IPAOI & $10.1180 \%$ & $12.4727 \%$ & $9.7792 \%$ & $12.8625 \%$ & $9.7804 \%$ & $12.8906 \%$ & $9.3457 \%$ & $12.4136 \%$ \\
\hline IPMVI & $2.5450 \%$ & $3.8648 \%$ & $2.3738 \%$ & $4.0981 \%$ & $3.2660 \%$ & $5.2596 \%$ & $3.0141 \%$ & $5.6165 \%$ \\
\hline PCB2C & & & & & $0.0015 \%$ & $0.3370 \%$ & $0.0003 \%$ & $0.4492 \%$ \\
\hline \multicolumn{9}{|l|}{ PCBBI } \\
\hline PCBDG & $0.1927 \%$ & $0.6891 \%$ & $0.1517 \%$ & $0.7971 \%$ & & & & \\
\hline PCBFA & & & & & $0.0015 \%$ & $0.3370 \%$ & $0.0003 \%$ & $0.4492 \%$ \\
\hline \multicolumn{9}{|l|}{ PCBFT } \\
\hline \multicolumn{9}{|l|}{ SYSBI } \\
\hline SYSFA & $0.7397 \%$ & $1.5393 \%$ & $0.6512 \%$ & $1.6932 \%$ & $0.7415 \%$ & $1.8647 \%$ & $0.6287 \%$ & $2.0905 \%$ \\
\hline SYSFT & $0.0569 \%$ & $0.4086 \%$ & $0.0378 \%$ & $0.4953 \%$ & $0.2094 \%$ & $0.9525 \%$ & $0.1559 \%$ & $1.1217 \%$ \\
\hline SYSPB & $0.0009 \%$ & $0.1952 \%$ & $0.0002 \%$ & $0.2602 \%$ & & & & \\
\hline
\end{tabular}


Table 13

\begin{tabular}{|c|c|c|c|c|c|c|c|c|}
\hline \multirow[t]{3}{*}{ Repaired Before Shipping } & \multicolumn{4}{|c|}{ Field Failure } & \multicolumn{4}{|c|}{ Field Failure } \\
\hline & \multicolumn{4}{|c|}{ Server Issue } & \multicolumn{4}{|c|}{ Hardware Failure } \\
\hline & \multicolumn{2}{|c|}{$95 \% \mathrm{Cl}$} & \multicolumn{2}{|c|}{$99 \% \mathrm{Cl}$} & \multicolumn{2}{|c|}{$95 \% \mathrm{Cl}$} & \multicolumn{2}{|c|}{$99 \% \mathrm{Cl}$} \\
\hline Test Area & $\mathrm{LCL}$ & $\mathrm{UCL}$ & LCL & $\mathrm{UCL}$ & $\mathrm{LCL}$ & $\mathrm{UCL}$ & $\mathrm{LCL}$ & $\mathrm{UCL}$ \\
\hline DBGHTHV & $0.0031 \%$ & $0.6727 \%$ & $0.0006 \%$ & $0.8960 \%$ & $0.0968 \%$ & $1.3637 \%$ & $0.0529 \%$ & $1.7046 \%$ \\
\hline ICT & $0.0031 \%$ & $0.6727 \%$ & $0.0006 \%$ & $0.8960 \%$ & & & & \\
\hline IPAOI & $9.7378 \%$ & $14.2673 \%$ & $9.1332 \%$ & $15.0459 \%$ & $10.4635 \%$ & $15.8212 \%$ & $9.7572 \%$ & $16.7457 \%$ \\
\hline IPMVI & $2.7658 \%$ & $5.5652 \%$ & $2.4487 \%$ & $6.0939 \%$ & $2.5436 \%$ & $5.7126 \%$ & $2.2041 \%$ & $6.3261 \%$ \\
\hline \multicolumn{9}{|l|}{ PCB2C } \\
\hline \multicolumn{9}{|l|}{ PCBBI } \\
\hline PCBDG & $0.0031 \%$ & $0.6727 \%$ & $0.0006 \%$ & $0.8960 \%$ & & & & \\
\hline \multicolumn{9}{|l|}{ PCBFA } \\
\hline PCBFT & & & & & $0.0040 \%$ & $0.8675 \%$ & $0.0008 \%$ & $1.1551 \%$ \\
\hline \multicolumn{9}{|l|}{ SYSBI } \\
\hline SYSFA & $0.4190 \%$ & $1.8994 \%$ & $0.3121 \%$ & $2.2348 \%$ & $0.2541 \%$ & $1.8137 \%$ & $0.1688 \%$ & $2.1951 \%$ \\
\hline SYSFT & $0.0750 \%$ & $1.0577 \%$ & $0.0409 \%$ & $1.3226 \%$ & $0.0379 \%$ & $1.1243 \%$ & $0.0162 \%$ & $1.4408 \%$ \\
\hline SYSPB & & & & & $0.0040 \%$ & $0.8675 \%$ & $0.0008 \%$ & $1.1551 \%$ \\
\hline
\end{tabular}

Table 14

\begin{tabular}{|l|r|r|r|r|r|r|r|r|}
\hline Repaired Before Shipping & \multicolumn{3}{|c|}{ Field Failure } & \multicolumn{4}{c|}{ Field Failure } \\
\hline & \multicolumn{3}{|c|}{ Memory Issue } & \multicolumn{3}{c|}{ CPU Failure } \\
\hline & \multicolumn{2}{|c|}{$95 \% \mathrm{Cl}$} & \multicolumn{2}{c|}{$99 \% \mathrm{Cl}$} & \multicolumn{2}{c|}{$95 \% \mathrm{Cl}$} & \multicolumn{2}{c|}{$99 \% \mathrm{Cl}$} \\
\hline Test Area & $\mathrm{LCL}$ & $\mathrm{UCL}$ & $\mathrm{LCL}$ & $\mathrm{UCL}$ & $\mathrm{LCL}$ & $\mathrm{UCL}$ & $\mathrm{LCL}$ & $\mathrm{UCL}$ \\
\hline DBGHTHV & $0.0620 \%$ & $1.8354 \%$ & $0.0265 \%$ & $2.3499 \%$ & $0.0701 \%$ & $0.0724 \%$ & $0.0300 \%$ & $2.6523 \%$ \\
\hline ICT & & & & & & & & \\
\hline IPAOI & $6.5320 \%$ & $12.5186 \%$ & $5.8300 \%$ & $13.6152 \%$ & $7.1475 \%$ & $13.7871 \%$ & $6.3698 \%$ & $15.0004 \%$ \\
\hline IPMVI & $1.0578 \%$ & $4.3244 \%$ & $0.8062 \%$ & $5.0430 \%$ & $1.5975 \%$ & $5.6172 \%$ & $1.2693 \%$ & $6.4720 \%$ \\
\hline PCB2C & & & & & $0.0073 \%$ & $1.5997 \%$ & $0.0014 \%$ & $2.1276 \%$ \\
\hline PCBBI & & & & & $0.0073 \%$ & $1.5997 \%$ & $0.0014 \%$ & $2.1276 \%$ \\
\hline PCBDG & $0.0065 \%$ & $1.4167 \%$ & $0.0013 \%$ & $1.8847 \%$ & & & & \\
\hline PCBFA & $0.1585 \%$ & $2.2258 \%$ & $0.0866 \%$ & $2.7790 \%$ & & & & \\
\hline PCBFT & $0.0065 \%$ & $1.4167 \%$ & $0.0013 \%$ & $1.8847 \%$ & & & & \\
\hline SYSBI & & & & & & & & \\
\hline SYSFA & & & & & $0.1792 \%$ & $2.5129 \%$ & $0.0979 \%$ & $3.1363 \%$ \\
\hline SYSFT & & & & & $0.0073 \%$ & $1.5997 \%$ & $0.0014 \%$ & $2.1276 \%$ \\
\hline SYSPB & & & & & & & & \\
\hline
\end{tabular}


Table 15

\begin{tabular}{|l|c|c|c|c|c|c|c|c|}
\hline Repaired Before Shipping & \multicolumn{3}{|c|}{ Field Failure } & \multicolumn{3}{c|}{ Field Failure } \\
\hline & \multicolumn{2}{|c|}{ Booting Issue } & \multicolumn{3}{c|}{ DOA } \\
\hline & \multicolumn{2}{|c|}{$95 \% \mathrm{Cl}$} & \multicolumn{2}{c|}{$99 \% \mathrm{Cl}$} & \multicolumn{2}{c|}{$95 \% \mathrm{Cl}$} & \multicolumn{2}{c|}{$99 \% \mathrm{Cl}$} \\
\hline Test Area & $\mathrm{LCL}$ & $\mathrm{UCL}$ & $\mathrm{LCL}$ & $\mathrm{UCL}$ & $\mathrm{LCL}$ & $\mathrm{UCL}$ & $\mathrm{LCL}$ & $\mathrm{UCL}$ \\
\hline DBGHTHV & & & & & & & & \\
\hline ICT & & & & & & & & \\
\hline IPAOI & $6.0354 \%$ & $12.8662 \%$ & $5.2752 \%$ & $14.1446 \%$ & $9.6450 \%$ & $18.5551 \%$ & $8.5917 \%$ & $20.1583 \%$ \\
\hline IPMVI & $1.6152 \%$ & $6.0645 \%$ & $1.2543 \%$ & $7.0207 \%$ & $1.6658 \%$ & $6.7503 \%$ & $1.2705 \%$ & $7.8552 \%$ \\
\hline PCB2C & & & & & & & & \\
\hline PCBBI & & & & & $0.0102 \%$ & $2.2172 \%$ & $0.0020 \%$ & $2.9458 \%$ \\
\hline PCBDG & $0.0811 \%$ & $2.3953 \%$ & $0.0347 \%$ & $3.0641 \%$ & $0.0102 \%$ & $2.2172 \%$ & $0.0020 \%$ & $2.9458 \%$ \\
\hline PCBFA & & & & & & & & \\
\hline PCBFT & $0.0085 \%$ & $1.8492 \%$ & $0.0017 \%$ & $2.4584 \%$ & & & & \\
\hline SYSBI & & & & & & & & \\
\hline SYSFA & $0.5451 \%$ & $3.8591 \%$ & $0.3623 \%$ & $4.6604 \%$ & $0.0102 \%$ & $2.2172 \%$ & $0.0020 \%$ & $2.9458 \%$ \\
\hline SYSFT & $0.0085 \%$ & $1.8492 \%$ & $0.0017 \%$ & $2.4584 \%$ & & & & \\
\hline SYSPB & & & & & & & & \\
\hline
\end{tabular}

\section{Table 16}

\begin{tabular}{|l|r|r|r|r|r|r|r|r|}
\hline Repaired After Shipping & \multicolumn{4}{|c|}{ Field Failure } & \multicolumn{4}{c|}{ Field Failure } \\
\hline & \multicolumn{3}{|c|}{ Motherboard Issue } & \multicolumn{3}{c|}{ Motherboard Failure } \\
\hline & \multicolumn{2}{|c|}{$95 \% \mathrm{Cl}$} & \multicolumn{2}{|c|}{$99 \% \mathrm{Cl}$} & \multicolumn{2}{c|}{$95 \% \mathrm{Cl}$} & \multicolumn{2}{c|}{$99 \% \mathrm{Cl}$} \\
\hline Test Area & $\mathrm{LCL}$ & $\mathrm{UCL}$ & $\mathrm{LCL}$ & $\mathrm{UCL}$ & $\mathrm{LCL}$ & $\mathrm{UCL}$ & $\mathrm{LCL}$ & $\mathrm{UCL}$ \\
\hline DGI & $9.0806 \%$ & $10.8517 \%$ & $8.8226 \%$ & $11.1432 \%$ & $6.4797 \%$ & $8.2798 \%$ & $6.2256 \%$ & $8.5820 \%$ \\
\hline FGI & $38.5463 \%$ & $41.4312 \%$ & $38.1010 \%$ & $41.8848 \%$ & $32.9673 \%$ & $36.2237 \%$ & $32.4695 \%$ & $36.7388 \%$ \\
\hline PRESCREEN & $49.0058 \%$ & $51.9498 \%$ & $48.5468 \%$ & $52.4083 \%$ & $42.7457 \%$ & $46.1463 \%$ & $42.2197 \%$ & $46.6785 \%$ \\
\hline SERVICE & $40.1375 \%$ & $43.0400 \%$ & $39.6888 \%$ & $43.4957 \%$ & $33.3523 \%$ & $36.6171 \%$ & $32.8529 \%$ & $37.1334 \%$ \\
\hline
\end{tabular}

Table 17

\begin{tabular}{|l|c|c|c|c|c|c|c|c|}
\hline Repaired After Shipping & \multicolumn{3}{|c|}{ Field Failure } & \multicolumn{3}{c|}{ Field Failure } \\
\hline & \multicolumn{3}{|c|}{ DIMM Issue } & \multicolumn{3}{c|}{ Blade Failure } \\
\hline & \multicolumn{2}{|c|}{$95 \% \mathrm{Cl}$} & \multicolumn{2}{c|}{$99 \% \mathrm{Cl}$} & \multicolumn{2}{c|}{$99 \% \mathrm{Cl}$} \\
\hline Test Area & $\mathrm{LCL}$ & $\mathrm{UCL}$ & $\mathrm{LCL}$ & $\mathrm{UCL}$ & $\mathrm{LCL}$ & $\mathrm{UCL}$ & $\mathrm{LCL}$ & $\mathrm{UCL}$ \\
\hline DGI & $5.7413 \%$ & $7.6027 \%$ & $5.4829 \%$ & $7.9184 \%$ & $7.9678 \%$ & $10.8340 \%$ & $7.5735 \%$ & $11.3211 \%$ \\
\hline FGI & $32.0992 \%$ & $35.6058 \%$ & $31.5649 \%$ & $36.1613 \%$ & $41.7417 \%$ & $46.5892 \%$ & $40.9976 \%$ & $47.3466 \%$ \\
\hline PRESCREEN & $38.9676 \%$ & $42.6082 \%$ & $38.4077 \%$ & $43.1801 \%$ & $50.3144 \%$ & $55.1876 \%$ & $49.5565 \%$ & $55.9393 \%$ \\
\hline SERVICE & $30.5438 \%$ & $34.0088 \%$ & $30.0171 \%$ & $34.5588 \%$ & $39.8198 \%$ & $44.6416 \%$ & $39.0819 \%$ & $45.3972 \%$ \\
\hline
\end{tabular}




\section{Table 18}

\begin{tabular}{|l|c|l|r|r|r|r|r|r|}
\hline Repaired After Shipping & \multicolumn{3}{|c|}{ Field Failure } & \multicolumn{3}{c|}{ Field Failure } \\
\hline & \multicolumn{3}{|c|}{ Server Issue } & \multicolumn{3}{c|}{ Hardware Failure } \\
\hline & \multicolumn{2}{|c|}{$95 \% \mathrm{Cl}$} & \multicolumn{2}{|c|}{$99 \% \mathrm{Cl}$} & \multicolumn{2}{c|}{$95 \% \mathrm{Cl}$} & \multicolumn{2}{c|}{$99 \% \mathrm{Cl}$} \\
\hline Test Area & $\mathrm{LCL}$ & $\mathrm{UCL}$ & $\mathrm{LCL}$ & $\mathrm{UCL}$ & $\mathrm{LCL}$ & $\mathrm{UCL}$ & $\mathrm{LCL}$ & $\mathrm{UCL}$ \\
\hline DGI & $7.6452 \%$ & $11.7772 \%$ & $7.1078 \%$ & $12.4993 \%$ & $7.5081 \%$ & $12.2468 \%$ & $6.9078 \%$ & $13.0850 \%$ \\
\hline FGI & $46.8969 \%$ & $53.8269 \%$ & $45.8301 \%$ & $54.8920 \%$ & $40.3272 \%$ & $48.1638 \%$ & $39.1431 \%$ & $49.3819 \%$ \\
\hline PRESCREEN & $55.5166 \%$ & $62.3369 \%$ & $54.4475 \%$ & $63.3653 \%$ & $49.1739 \%$ & $57.0472 \%$ & $47.9579 \%$ & $58.2248 \%$ \\
\hline SERVICE & $44.3667 \%$ & $51.2905 \%$ & $43.3067 \%$ & $52.3604 \%$ & $39.7111 \%$ & $47.5358 \%$ & $38.5306 \%$ & $48.7538 \%$ \\
\hline
\end{tabular}

\section{Table 19}

\begin{tabular}{|l|r|l|r|l|r|r|r|r|}
\hline Repaired After Shipping & \multicolumn{4}{|c|}{ Field Failure } & \multicolumn{4}{c|}{ Field Failure } \\
\hline & \multicolumn{3}{|c|}{ Memory Issue } & \multicolumn{3}{c|}{ CPU Failure } \\
\hline & \multicolumn{2}{|c|}{$95 \% \mathrm{Cl}$} & \multicolumn{2}{c|}{$99 \% \mathrm{Cl}$} & \multicolumn{2}{c|}{$95 \% \mathrm{Cl}$} & \multicolumn{3}{c|}{$99 \% \mathrm{Cl}$} \\
\hline Test Area & $\mathrm{LCL}$ & $\mathrm{UCL}$ & $\mathrm{LCL}$ & $\mathrm{UCL}$ & $\mathrm{LCL}$ & $\mathrm{UCL}$ & $\mathrm{LCL}$ & $\mathrm{UCL}$ \\
\hline DGI & $5.8808 \%$ & $11.6486 \%$ & $5.2162 \%$ & $12.7155 \%$ & $5.4446 \%$ & $11.4836 \%$ & $4.7705 \%$ & $12.6163 \%$ \\
\hline FGI & $32.5296 \%$ & $42.3439 \%$ & $31.0997 \%$ & $43.8953 \%$ & $50.0822 \%$ & $60.8056 \%$ & $48.4272 \%$ & $62.4012 \%$ \\
\hline PRESCREEN & $41.2658 \%$ & $51.3739 \%$ & $39.7475 \%$ & $52.9278 \%$ & $56.2092 \%$ & $66.7125 \%$ & $54.5554 \%$ & $68.2411 \%$ \\
\hline SERVICE & $31.2954 \%$ & $41.0402 \%$ & $29.8824 \%$ & $42.5869 \%$ & $40.0447 \%$ & $50.7865 \%$ & $38.4416 \%$ & $52.4397 \%$ \\
\hline
\end{tabular}

Table 20

\begin{tabular}{|l|c|l|r|l|r|r|r|r|}
\hline Repaired After Shipping & \multicolumn{3}{|c|}{ Field Failure } & \multicolumn{4}{c|}{ Field Failure } \\
\hline & \multicolumn{3}{|c|}{ Booting Issue } & \multicolumn{2}{c|}{$95 \% \mathrm{Cl}$} & \multicolumn{2}{c|}{$99 \% \mathrm{Cl}$} \\
\hline & \multicolumn{2}{|c|}{$95 \% \mathrm{Cl}$} & \multicolumn{2}{|c|}{$99 \% \mathrm{Cl}$} & $\mathrm{UCL}$ & $\mathrm{LCL}$ & $\mathrm{UCL}$ \\
\hline Test Area & $\mathrm{LCL}$ & $\mathrm{UCL}$ & $\mathrm{LCL}$ & $\mathrm{UCL}$ & $\mathrm{LCL}$ & $\mathrm{U}$ \\
\hline DGI & $9.4422 \%$ & $17.3970 \%$ & $8.4838 \%$ & $18.8214 \%$ & $5.6200 \%$ & $13.0712 \%$ & $4.8265 \%$ & $14.4898 \%$ \\
\hline FGI & $42.7025 \%$ & $54.3176 \%$ & $40.9573 \%$ & $56.0816 \%$ & $36.3493 \%$ & $48.9703 \%$ & $34.5082 \%$ & $50.9231 \%$ \\
\hline PRESCREEN & $53.3910 \%$ & $64.8195 \%$ & $51.6076 \%$ & $66.4876 \%$ & $43.0265 \%$ & $55.7832 \%$ & $41.1133 \%$ & $57.7054 \%$ \\
\hline SERVICE & $43.6935 \%$ & $55.3132 \%$ & $41.9414 \%$ & $57.0716 \%$ & $32.8620 \%$ & $45.3159 \%$ & $31.0738 \%$ & $47.2701 \%$ \\
\hline
\end{tabular}


Table 21

\begin{tabular}{|c|c|c|c|c|c|c|c|c|}
\hline \multirow{4}{*}{\begin{tabular}{|l|} 
Passed Before Shipping \\
\\
Test Area \\
\end{tabular}} & \multicolumn{4}{|c|}{ Field Failure } & \multicolumn{4}{|c|}{ Field Failure } \\
\hline & \multicolumn{4}{|c|}{ Motherboard Issue } & \multicolumn{4}{|c|}{ Motherboard Failure } \\
\hline & \multicolumn{2}{|c|}{$95 \% \mathrm{Cl}$} & \multicolumn{2}{|c|}{$99 \% \mathrm{Cl}$} & \multicolumn{2}{|c|}{$95 \% \mathrm{Cl}$} & \multicolumn{2}{|c|}{$99 \% \mathrm{Cl}$} \\
\hline & $\mathrm{LCL}$ & UCL & $\mathrm{LCL}$ & UCL & $\mathrm{LCL}$ & UCL & $\mathrm{LCL}$ & UCL \\
\hline ASSY & $68.4816 \%$ & $71.1857 \%$ & $68.0522 \%$ & $71.5986 \%$ & $68.7809 \%$ & $71.9086 \%$ & $68.2831 \%$ & $72.3835 \%$ \\
\hline DBGHTHV & $35.0600 \%$ & $37.8951 \%$ & $34.6239 \%$ & $38.3424 \%$ & $31.3693 \%$ & $34.5881 \%$ & $30.8783 \%$ & $35.0982 \%$ \\
\hline DBGHTLV & $0.0006 \%$ & $0.1238 \%$ & $0.0001 \%$ & $0.1651 \%$ & & & & \\
\hline DBGLTLV & $3.3246 \%$ & $4.4748 \%$ & $3.1665 \%$ & $4.6715 \%$ & $3.0451 \%$ & $4.3497 \%$ & $2.8708 \%$ & $4.5764 \%$ \\
\hline \multicolumn{9}{|c|}{ 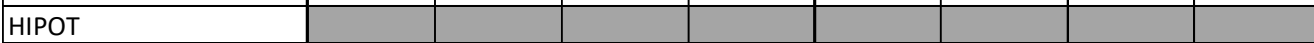 } \\
\hline ICT & $98.6887 \%$ & $99.2882 \%$ & $98.5767 \%$ & $99.3588 \%$ & $98.7543 \%$ & $99.4172 \%$ & $98.6257 \%$ & $99.4896 \%$ \\
\hline OSYSBI* & $0.0054 \%$ & $0.1606 \%$ & $0.0023 \%$ & $0.2061 \%$ & & & & \\
\hline \begin{tabular}{|l|} 
PASTE \\
\end{tabular} & $98.6633 \%$ & $99.2692 \%$ & $98.5503 \%$ & $99.3408 \%$ & $98.7896 \%$ & $99.4417 \%$ & $98.6627 \%$ & $99.5124 \%$ \\
\hline PCB2C & $70.1253 \%$ & $72.7865 \%$ & $69.7020 \%$ & $73.1919 \%$ & $73.4714 \%$ & $76.4397 \%$ & $72.9961 \%$ & $76.8870 \%$ \\
\hline PCBBI & $3.4901 \%$ & $4.6648 \%$ & $3.3281 \%$ & $4.8653 \%$ & $2.9629 \%$ & $4.2523 \%$ & $2.7910 \%$ & $4.4767 \%$ \\
\hline \multicolumn{9}{|l|}{ PCBBI* } \\
\hline \multicolumn{9}{|l|}{ PCBBS } \\
\hline PCBCEV & $0.5057 \%$ & $1.0290 \%$ & $0.4471 \%$ & $1.1293 \%$ & $2.2822 \%$ & $3.4363 \%$ & $2.1319 \%$ & $3.6400 \%$ \\
\hline PCBDG & & & & & $0.0008 \%$ & $0.1669 \%$ & $0.0002 \%$ & $0.2224 \%$ \\
\hline PCBDL & $2.9946 \%$ & $4.0938 \%$ & $2.8447 \%$ & $4.2826 \%$ & $2.4719 \%$ & $3.6656 \%$ & $2.3153 \%$ & $3.8754 \%$ \\
\hline PCBDL* & & & & & $0.0008 \%$ & $0.1669 \%$ & $0.0002 \%$ & $0.2224 \%$ \\
\hline PCBFA & $1.1957 \%$ & $1.9379 \%$ & $1.1026 \%$ & $2.0718 \%$ & $0.8577 \%$ & $1.6287 \%$ & $0.7683 \%$ & $1.7738 \%$ \\
\hline PCBFA* & & & & & $0.0008 \%$ & $0.1669 \%$ & $0.0002 \%$ & $0.2224 \%$ \\
\hline \begin{tabular}{|l|} 
PCBFT \\
\end{tabular} & $98.6887 \%$ & $99.2882 \%$ & $98.5767 \%$ & $99.3588 \%$ & $98.6840 \%$ & $99.3680 \%$ & $98.5522 \%$ & $99.4436 \%$ \\
\hline PCBINT & $3.5522 \%$ & $4.7360 \%$ & $3.3888 \%$ & $4.9379 \%$ & $5.9714 \%$ & $7.7103 \%$ & $5.7272 \%$ & $8.0032 \%$ \\
\hline \begin{tabular}{|l|} 
PCBP2 \\
\end{tabular} & $0.5613 \%$ & $1.1066 \%$ & $0.4993 \%$ & $1.2102 \%$ & $0.2076 \%$ & $0.6653 \%$ & $0.1674 \%$ & $0.7626 \%$ \\
\hline PCBPB & $3.8428 \%$ & $5.0677 \%$ & $3.6727 \%$ & $5.2758 \%$ & $3.4026 \%$ & $4.7705 \%$ & $3.2182 \%$ & $5.0069 \%$ \\
\hline PCBPB* & $0.0054 \%$ & $0.1606 \%$ & $0.0023 \%$ & $0.2061 \%$ & & & & \\
\hline \multicolumn{9}{|l|}{ PCBPEP } \\
\hline PCBPM & $98.3859 \%$ & $99.0580 \%$ & $98.2628 \%$ & $99.1400 \%$ & $98.4749 \%$ & $99.2181 \%$ & $98.3341 \%$ & $99.3032 \%$ \\
\hline \begin{tabular}{|l|} 
PCBST \\
\end{tabular} & $41.3984 \%$ & $44.3125 \%$ & $40.9473 \%$ & $44.7695 \%$ & $42.9545 \%$ & $46.3566 \%$ & $42.4282 \%$ & $46.8890 \%$ \\
\hline POSTBI & $3.2213 \%$ & $4.3559 \%$ & $3.0657 \%$ & $4.5502 \%$ & $2.6896 \%$ & $3.9269 \%$ & $2.5260 \%$ & $4.1434 \%$ \\
\hline SYSADBG & $0.0242 \%$ & $0.2276 \%$ & $0.0150 \%$ & $0.2798 \%$ & & & & \\
\hline SYSAPK & $1.0002 \%$ & $1.6891 \%$ & $0.9155 \%$ & $1.8149 \%$ & $2.5806 \%$ & $3.7964 \%$ & $2.4205 \%$ & $4.0096 \%$ \\
\hline SYSAPK* & $11.3079 \%$ & $13.2466 \%$ & $11.0226 \%$ & $13.5634 \%$ & $14.2809 \%$ & $16.7658 \%$ & $13.9148 \%$ & $17.1706 \%$ \\
\hline SYSBI & $0.3780 \%$ & $0.8460 \%$ & $0.3282 \%$ & $0.9378 \%$ & $0.3665 \%$ & $0.9241 \%$ & $0.3107 \%$ & $1.0366 \%$ \\
\hline \multicolumn{9}{|l|}{ SYSBI* } \\
\hline SYSCEV & $0.0626 \%$ & $0.3205 \%$ & $0.0453 \%$ & $0.3806 \%$ & $0.2295 \%$ & $0.7029 \%$ & $0.1869 \%$ & $0.8026 \%$ \\
\hline SYSCEV* & $0.2374 \%$ & $0.6319 \%$ & $0.1991 \%$ & $0.7125 \%$ & $1.5329 \%$ & $2.5095 \%$ & $1.4108 \%$ & $2.6860 \%$ \\
\hline SYSDL & $0.0626 \%$ & $0.3205 \%$ & $0.0453 \%$ & $0.3806 \%$ & $0.1036 \%$ & $0.4718 \%$ & $0.0771 \%$ & $0.5559 \%$ \\
\hline SYSFA & $85.4457 \%$ & $87.4665 \%$ & $85.1166 \%$ & $87.7651 \%$ & $81.7524 \%$ & $84.3266 \%$ & $81.3343 \%$ & $84.7076 \%$ \\
\hline SYSFA* & & & & & $0.0008 \%$ & $0.1669 \%$ & $0.0002 \%$ & $0.2224 \%$ \\
\hline \begin{tabular}{|l|} 
SYSFT* \\
\end{tabular} & $86.3171 \%$ & $88.2839 \%$ & $85.9961 \%$ & $88.5738 \%$ & $82.6472 \%$ & $85.1683 \%$ & $82.2370 \%$ & $85.5404 \%$ \\
\hline SYSINT & $68.2790 \%$ & $70.9883 \%$ & $67.8490 \%$ & $71.4020 \%$ & $62.4451 \%$ & $65.7291 \%$ & $61.9264 \%$ & $66.2320 \%$ \\
\hline SYSINT* & & & & & $0.0008 \%$ & $0.1669 \%$ & $0.0002 \%$ & $0.2224 \%$ \\
\hline SYSPB & $1.1173 \%$ & $1.8386 \%$ & $1.0274 \%$ & $1.9694 \%$ & $1.0882 \%$ & $1.9367 \%$ & $0.9866 \%$ & $2.0936 \%$ \\
\hline SYSPEP & $69.0218 \%$ & $71.7122 \%$ & $68.5943 \%$ & $72.1227 \%$ & $63.4736 \%$ & $66.7366 \%$ & $62.9577 \%$ & $67.2356 \%$ \\
\hline SYSPM & $83.7294 \%$ & $85.8493 \%$ & $83.3855 \%$ & $86.1643 \%$ & $79.8739 \%$ & $82.5523 \%$ & $79.4406 \%$ & $82.9506 \%$ \\
\hline SYSVF & $5.1383 \%$ & $6.5276 \%$ & $4.9419 \%$ & $6.7609 \%$ & $4.5961 \%$ & $6.1516 \%$ & $4.3815 \%$ & $6.4166 \%$ \\
\hline \multicolumn{9}{|l|}{\begin{tabular}{|l|} 
UNKNOWN \\
\end{tabular}} \\
\hline \multicolumn{9}{|l|}{ WPCBAPK } \\
\hline WPCBASIC1 & & & & & $0.0008 \%$ & $0.1669 \%$ & $0.0002 \%$ & $0.2224 \%$ \\
\hline WPCBASIC3 & $0.0054 \%$ & $0.1606 \%$ & $0.0023 \%$ & $0.2061 \%$ & $0.0073 \%$ & $0.2163 \%$ & $0.0031 \%$ & $0.2776 \%$ \\
\hline XRAY & $22.5990 \%$ & $25.1113 \%$ & $22.2188 \%$ & $25.5132 \%$ & $24.3826 \%$ & $27.3829 \%$ & $23.9298 \%$ & $27.8628 \%$ \\
\hline XTD4C & $0.0138 \%$ & $0.1948 \%$ & $0.0075 \%$ & $0.2439 \%$ & $0.0185 \%$ & $0.2625 \%$ & $0.0101 \%$ & $0.3286 \%$ \\
\hline XTDSNT & $1.0391 \%$ & $1.7391 \%$ & $0.9527 \%$ & $1.8665 \%$ & $0.7819 \%$ & $1.5251 \%$ & $0.6968 \%$ & $1.6659 \%$ \\
\hline ZSYSBI & & & & & & & & \\
\hline
\end{tabular}


Table 22

\begin{tabular}{|c|c|c|c|c|c|c|c|c|}
\hline \multirow[t]{3}{*}{ Passed Before Shipping } & \multicolumn{4}{|c|}{ Field Failure } & \multicolumn{4}{|c|}{ Field Failure } \\
\hline & \multicolumn{4}{|c|}{ DIMM Issue } & \multicolumn{4}{|c|}{ Blade Failure } \\
\hline & \multicolumn{2}{|c|}{$95 \% \mathrm{Cl}$} & \multicolumn{2}{|c|}{$99 \% \mathrm{Cl}$} & \multicolumn{2}{|c|}{$95 \% \mathrm{Cl}$} & \multicolumn{2}{|c|}{$99 \% \mathrm{Cl}$} \\
\hline Test Area & $L C L$ & $\mathrm{UCL}$ & $\mathrm{LCL}$ & UCL & $\mathrm{LCL}$ & $\mathrm{UCL}$ & $\mathrm{LCL}$ & $\mathrm{UCL}$ \\
\hline ASSY & $76.9164 \%$ & $79.9674 \%$ & $76.4243 \%$ & $80.4222 \%$ & $55.8459 \%$ & $60.6601 \%$ & $55.0909 \%$ & $61.3963 \%$ \\
\hline DBGHTHV & $25.0668 \%$ & $28.3472 \%$ & $24.5727 \%$ & $28.8720 \%$ & $35.0316 \%$ & $39.7560 \%$ & $34.3144 \%$ & $40.5018 \%$ \\
\hline \multicolumn{9}{|l|}{ DBGHTLV } \\
\hline DBGLTLV & $1.7324 \%$ & $2.8567 \%$ & $1.5923 \%$ & $3.0601 \%$ & $6.5645 \%$ & $9.2147 \%$ & $6.2057 \%$ & $9.6698 \%$ \\
\hline \multicolumn{9}{|c|}{ 每 } \\
\hline ICT & $95.3318 \%$ & $96.7877 \%$ & $95.0777 \%$ & $96.9807 \%$ & $96.8544 \%$ & $98.3662 \%$ & $96.5701 \%$ & $98.5407 \%$ \\
\hline OSYSBI* & $0.0009 \%$ & $0.1952 \%$ & $0.0002 \%$ & $0.2602 \%$ & & & & \\
\hline PASTE & $98.1757 \%$ & $99.0554 \%$ & $98.0096 \%$ & $99.1566 \%$ & $96.7154 \%$ & $98.2636 \%$ & $96.4255 \%$ & $98.4439 \%$ \\
\hline PCB2C & $66.4530 \%$ & $69.9051 \%$ & $65.9047 \%$ & $70.4295 \%$ & $75.2500 \%$ & $79.3464 \%$ & $74.5858 \%$ & $79.9485 \%$ \\
\hline PCBBI & $1.5478 \%$ & $2.6211 \%$ & $1.4159 \%$ & $2.8168 \%$ & $7.1804 \%$ & $9.9288 \%$ & $6.8055 \%$ & $10.3984 \%$ \\
\hline PCBBI* & $0.0009 \%$ & $0.1952 \%$ & $0.0002 \%$ & $0.2602 \%$ & & & & \\
\hline \multicolumn{9}{|l|}{ PCBBS } \\
\hline PCBCEV & $1.2133 \%$ & $2.1855 \%$ & $1.0975 \%$ & $2.3657 \%$ & $1.4809 \%$ & $2.9361 \%$ & $1.3155 \%$ & $3.2118 \%$ \\
\hline \multicolumn{9}{|c|}{ 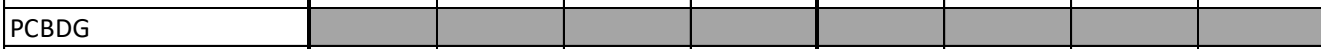 } \\
\hline PCBDL & $1.0634 \%$ & $1.9854 \%$ & $0.9555 \%$ & $2.1580 \%$ & $7.3488 \%$ & $10.1231 \%$ & $6.9697 \%$ & $10.5965 \%$ \\
\hline PCBDL* & $0.0085 \%$ & $0.2531 \%$ & $0.0036 \%$ & $0.3248 \%$ & & & & \\
\hline PCBFA & $0.2947 \%$ & $0.8660 \%$ & $0.2420 \%$ & $0.9852 \%$ & $4.4607 \%$ & $6.7244 \%$ & $4.1650 \%$ & $7.1218 \%$ \\
\hline \multicolumn{9}{|c|}{ 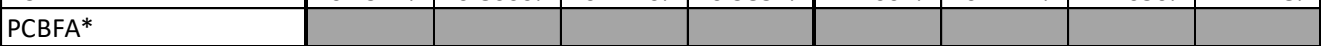 } \\
\hline PCBFT & $92.0994 \%$ & $93.9962 \%$ & $91.7784 \%$ & $94.2603 \%$ & $96.8544 \%$ & $98.3662 \%$ & $96.5701 \%$ & $98.5407 \%$ \\
\hline PCBINT & $3.7256 \%$ & $5.2757 \%$ & $3.5174 \%$ & $5.5441 \%$ & $8.5891 \%$ & $11.5428 \%$ & $8.1803 \%$ & $12.0428 \%$ \\
\hline PCBP2 & $0.2429 \%$ & $0.7782 \%$ & $0.1959 \%$ & $0.8920 \%$ & $1.3798 \%$ & $2.7957 \%$ & $1.2206 \%$ & $3.0655 \%$ \\
\hline PCBPB & $1.7324 \%$ & $2.8567 \%$ & $1.5923 \%$ & $3.0601 \%$ & $9.2690 \%$ & $12.3138 \%$ & $8.8452 \%$ & $12.8272 \%$ \\
\hline PCBPB* & $0.0009 \%$ & $0.1952 \%$ & $0.0002 \%$ & $0.2602 \%$ & & & & \\
\hline PCBPEP & & & & & $0.0147 \%$ & $0.4369 \%$ & $0.0063 \%$ & $0.5605 \%$ \\
\hline PCBPM & $98.1757 \%$ & $99.0554 \%$ & $98.0096 \%$ & $99.1566 \%$ & $94.8075 \%$ & $96.7877 \%$ & $94.4526 \%$ & $97.0374 \%$ \\
\hline PCBST & $44.6850 \%$ & $48.3797 \%$ & $44.1129 \%$ & $48.9563 \%$ & $30.0904 \%$ & $34.6607 \%$ & $29.4031 \%$ & $35.3880 \%$ \\
\hline POSTBI & $1.4560 \%$ & $2.5028 \%$ & $1.3283 \%$ & $2.6945 \%$ & $6.3970 \%$ & $9.0195 \%$ & $6.0427 \%$ & $9.4705 \%$ \\
\hline SYSADBG & & & & & $0.9336 \%$ & $2.1553 \%$ & $0.8052 \%$ & $2.3958 \%$ \\
\hline SYSAPK & $1.1232 \%$ & $2.0656 \%$ & $1.0120 \%$ & $2.2413 \%$ & $0.2908 \%$ & $1.1111 \%$ & $0.2255 \%$ & $1.2916 \%$ \\
\hline SYSAPK* & $14.1881 \%$ & $16.8789 \%$ & $13.7938 \%$ & $17.3185 \%$ & $9.3258 \%$ & $12.3780 \%$ & $8.9007 \%$ & $12.8924 \%$ \\
\hline SYSBI & $0.0217 \%$ & $0.3071 \%$ & $0.0118 \%$ & $0.3844 \%$ & $2.9444 \%$ & $4.8560 \%$ & $2.7058 \%$ & $5.2005 \%$ \\
\hline SYSBI* & & & & & $0.0984 \%$ & $0.7053 \%$ & $0.0653 \%$ & $0.8647 \%$ \\
\hline SYSCEV & $0.0987 \%$ & $0.5050 \%$ & $0.0715 \%$ & $0.5997 \%$ & $0.1335 \%$ & $0.7893 \%$ & $0.0932 \%$ & $0.9457 \%$ \\
\hline SYSCEV* & $1.0038 \%$ & $1.9050 \%$ & $0.8992 \%$ & $2.0744 \%$ & $1.0312 \%$ & $2.2990 \%$ & $0.8956 \%$ & $2.5465 \%$ \\
\hline SYSDL & $0.0382 \%$ & $0.3587 \%$ & $0.0236 \%$ & $0.4409 \%$ & $0.0661 \%$ & $0.6192 \%$ & $0.0407 \%$ & $0.7608 \%$ \\
\hline SYSFA & $70.7606 \%$ & $74.0741 \%$ & $70.2312 \%$ & $74.5739 \%$ & $84.1139 \%$ & $87.5321 \%$ & $83.5461 \%$ & $88.0182 \%$ \\
\hline \multicolumn{9}{|l|}{ SYSFA* } \\
\hline SYSFT* & $82.3604 \%$ & $85.1015 \%$ & $81.9133 \%$ & $85.5042 \%$ & $85.2580 \%$ & $88.5644 \%$ & $84.7064 \%$ & $89.0319 \%$ \\
\hline SYSINT & $64.8908 \%$ & $68.3849 \%$ & $64.3369 \%$ & $68.9169 \%$ & $71.7045 \%$ & $75.9995 \%$ & $71.0131 \%$ & $76.6366 \%$ \\
\hline \multicolumn{9}{|l|}{\begin{tabular}{|l|} 
SYSINT* \\
\end{tabular}} \\
\hline SYSPB & $0.4016 \%$ & $1.0384 \%$ & $0.3387 \%$ & $1.1676 \%$ & $4.4607 \%$ & $6.7244 \%$ & $4.1650 \%$ & $7.1218 \%$ \\
\hline SYSPEP & $65.4231 \%$ & $68.9034 \%$ & $64.8711 \%$ & $69.4329 \%$ & $72.6979 \%$ & $76.9408 \%$ & $72.0136 \%$ & $77.5686 \%$ \\
\hline SYSPM & $80.3363 \%$ & $83.2026 \%$ & $79.8709 \%$ & $83.6262 \%$ & $81.4570 \%$ & $85.1104 \%$ & $80.8553 \%$ & $85.6363 \%$ \\
\hline SYSVF & $4.5988 \%$ & $6.2936 \%$ & $4.3674 \%$ & $6.5840 \%$ & $2.9979 \%$ & $4.9234 \%$ & $2.7570 \%$ & $5.2701 \%$ \\
\hline UNKNOWN & $0.0009 \%$ & $0.1952 \%$ & $0.0002 \%$ & $0.2602 \%$ & $0.0015 \%$ & $0.3370 \%$ & $0.0003 \%$ & $0.4492 \%$ \\
\hline WPCBAPK & $0.0009 \%$ & $0.1952 \%$ & $0.0002 \%$ & $0.2602 \%$ & & & & \\
\hline WPCBASIC1 & $0.0009 \%$ & $0.1952 \%$ & $0.0002 \%$ & $0.2602 \%$ & $0.0015 \%$ & $0.3370 \%$ & $0.0003 \%$ & $0.4492 \%$ \\
\hline \multicolumn{9}{|l|}{ WPCBASIC3 } \\
\hline XRAY & $15.8810 \%$ & $18.6886 \%$ & $15.4673 \%$ & $19.1454 \%$ & $33.9576 \%$ & $38.6533 \%$ & $33.2462 \%$ & $39.3959 \%$ \\
\hline XTD4C & $0.0009 \%$ & $0.1952 \%$ & $0.0002 \%$ & $0.2602 \%$ & $0.0015 \%$ & $0.3370 \%$ & $0.0003 \%$ & $0.4492 \%$ \\
\hline XTDSNT & $0.1212 \%$ & $0.5520 \%$ & $0.0902 \%$ & $0.6502 \%$ & $1.9430 \%$ & $3.5613 \%$ & $1.7514 \%$ & $3.8617 \%$ \\
\hline ZSYSBI & & & & & $0.0147 \%$ & $0.4369 \%$ & $0.0063 \%$ & $0.5605 \%$ \\
\hline
\end{tabular}


Table 23

\begin{tabular}{|c|c|c|c|c|c|c|c|c|}
\hline \multirow[t]{3}{*}{ Passed Before Shipping } & \multicolumn{4}{|c|}{ Field Failure } & \multicolumn{4}{|c|}{ Field Failure } \\
\hline & \multicolumn{4}{|c|}{ Server Issue } & \multicolumn{4}{|c|}{ Hardware Failure } \\
\hline & \multicolumn{2}{|c|}{$95 \% \mathrm{Cl}$} & \multicolumn{2}{|c|}{$99 \% \mathrm{Cl}$} & \multicolumn{2}{|c|}{$95 \% \mathrm{Cl}$} & \multicolumn{2}{|c|}{$99 \% \mathrm{Cl}$} \\
\hline Test Area & LCL & UCL & LCL & UCL & $\mathrm{LCL}$ & UCL & LCL & $\mathrm{UCL}$ \\
\hline ASSY & $57.7184 \%$ & $64.4778 \%$ & $56.6538 \%$ & $65.4918 \%$ & $54.6672 \%$ & $62.4407 \%$ & $53.4507 \%$ & $63.6068 \%$ \\
\hline DBGHTHV & $34.9287 \%$ & $41.6688 \%$ & $33.9191 \%$ & $42.7217 \%$ & $39.8650 \%$ & $47.6929 \%$ & $38.6836 \%$ & $48.9109 \%$ \\
\hline \multicolumn{9}{|l|}{ DBGHTLV } \\
\hline DBGLTLV & $4.6314 \%$ & $8.0385 \%$ & $4.2148 \%$ & $8.6561 \%$ & $3.8384 \%$ & $7.5239 \%$ & $3.4142 \%$ & $8.2121 \%$ \\
\hline HIPOT & & & & & $0.0040 \%$ & $0.8675 \%$ & $0.0008 \%$ & $1.1551 \%$ \\
\hline ICT & $97.1726 \%$ & $99.0704 \%$ & $96.7763 \%$ & $99.2426 \%$ & $97.5519 \%$ & $99.4588 \%$ & $97.1211 \%$ & $99.5969 \%$ \\
\hline \multicolumn{9}{|l|}{ OSYSBI* } \\
\hline PASTE & $97.1726 \%$ & $99.0704 \%$ & $96.7763 \%$ & $99.2426 \%$ & $97.3473 \%$ & $99.3550 \%$ & $96.9020 \%$ & $99.5087 \%$ \\
\hline PCB2C & $75.9923 \%$ & $81.6687 \%$ & $75.0562 \%$ & $82.4732 \%$ & $70.9693 \%$ & $77.8651 \%$ & $69.8427 \%$ & $78.8478 \%$ \\
\hline PCBBI & $3.8968 \%$ & $7.0854 \%$ & $3.5162 \%$ & $7.6711 \%$ & $3.8384 \%$ & $7.5239 \%$ & $3.4142 \%$ & $8.2121 \%$ \\
\hline \multicolumn{9}{|l|}{ PCBBI* } \\
\hline \multicolumn{9}{|l|}{ PCBBS } \\
\hline PCBCEV & $1.2965 \%$ & $3.4222 \%$ & $1.0881 \%$ & $3.8520 \%$ & $0.8610 \%$ & $3.0545 \%$ & $0.6782 \%$ & $3.5266 \%$ \\
\hline \multicolumn{9}{|l|}{ PCBDG } \\
\hline PCBDL & $3.0709 \%$ & $5.9830 \%$ & $2.7355 \%$ & $6.5282 \%$ & $3.7068 \%$ & $7.3449 \%$ & $3.2904 \%$ & $8.0262 \%$ \\
\hline \multicolumn{9}{|l|}{ PCBDL* } \\
\hline PCBFA & $0.8406 \%$ & $2.6763 \%$ & $0.6782 \%$ & $3.0634 \%$ & $1.6752 \%$ & $4.4086 \%$ & $1.4063 \%$ & $4.9590 \%$ \\
\hline \multicolumn{9}{|l|}{ PCBFA* } \\
\hline PCBFT & $97.1726 \%$ & $99.0704 \%$ & $96.7763 \%$ & $99.2426 \%$ & $97.5519 \%$ & $99.4588 \%$ & $97.1211 \%$ & $99.5969 \%$ \\
\hline PCBINT & $5.6957 \%$ & $9.3855 \%$ & $5.2323 \%$ & $10.0441 \%$ & $4.7702 \%$ & $8.7674 \%$ & $4.2945 \%$ & $9.5003 \%$ \\
\hline PCBP2 & $0.3414 \%$ & $1.7382 \%$ & $0.2472 \%$ & $2.0616 \%$ & $0.2541 \%$ & $1.8137 \%$ & $0.1688 \%$ & $2.1951 \%$ \\
\hline PCBPB & $5.3748 \%$ & $8.9830 \%$ & $4.9249 \%$ & $9.6297 \%$ & $5.3099 \%$ & $9.4710 \%$ & $4.8068 \%$ & $10.2273 \%$ \\
\hline \multicolumn{9}{|l|}{ PCBPB* } \\
\hline PCBPEP & & & & & & & & \\
\hline PCBPM & $96.5778 \%$ & $98.7035 \%$ & $96.1480 \%$ & 98.9119\% & $96.9455 \%$ & $99.1390 \%$ & $96.4734 \%$ & $99.3218 \%$ \\
\hline PCBST & $33.2698 \%$ & $39.9499 \%$ & $32.2734 \%$ & $41.0074 \%$ & $27.3732 \%$ & $34.6787 \%$ & $26.3107 \%$ & $35.8530 \%$ \\
\hline POSTBI & $4.1057 \%$ & $7.3587 \%$ & $3.7145 \%$ & $7.9538 \%$ & $3.3145 \%$ & $6.8051 \%$ & $2.9224 \%$ & $7.4652 \%$ \\
\hline SYSADBG & $0.0293 \%$ & $0.8719 \%$ & $0.0125 \%$ & $1.1178 \%$ & $0.0040 \%$ & $0.8675 \%$ & $0.0008 \%$ & $1.1551 \%$ \\
\hline SYSAPK & $0.5820 \%$ & $2.2151 \%$ & $0.4514 \%$ & $2.5727 \%$ & $1.7966 \%$ & $4.5974 \%$ & $1.5169 \%$ & $5.1575 \%$ \\
\hline SYSAPK* & $10.6274 \%$ & $15.3075 \%$ & $9.9971 \%$ & $16.1073 \%$ & $8.9072 \%$ & $13.9570 \%$ & $8.2539 \%$ & $14.8388 \%$ \\
\hline SYSBI & $0.4190 \%$ & $1.8994 \%$ & $0.3121 \%$ & $2.2348 \%$ & $1.0859 \%$ & $3.4485 \%$ & $0.8763 \%$ & $3.9450 \%$ \\
\hline SYSBI* & & & & & & & & \\
\hline SYSCEV & $0.0293 \%$ & $0.8719 \%$ & $0.0125 \%$ & $1.1178 \%$ & $0.1705 \%$ & $1.5925 \%$ & $0.1052 \%$ & $1.9547 \%$ \\
\hline SYSCEV* & $1.1111 \%$ & $3.1266 \%$ & $0.9203 \%$ & $3.5401 \%$ & $0.5412 \%$ & $2.4481 \%$ & $0.4031 \%$ & $2.8789 \%$ \\
\hline SYSDL & & & & & $0.0040 \%$ & $0.8675 \%$ & $0.0008 \%$ & $1.1551 \%$ \\
\hline SYSFA & $82.1098 \%$ & $87.1308 \%$ & $81.2625 \%$ & $87.8198 \%$ & $83.0003 \%$ & $88.5381 \%$ & $82.0512 \%$ & $89.2757 \%$ \\
\hline SYSFA* & & & & & & & & \\
\hline SYSFT* & $83.1401 \%$ & $88.0304 \%$ & $82.3109 \%$ & $88.6968 \%$ & $85.0241 \%$ & $90.2459 \%$ & $84.1184 \%$ & $90.9288 \%$ \\
\hline SYSINT & $62.3898 \%$ & $68.9744 \%$ & $61.3420 \%$ & $69.9507 \%$ & $53.2501 \%$ & $61.0583 \%$ & $52.0324 \%$ & $62.2339 \%$ \\
\hline SYSINT* & & & & & & & & \\
\hline SYSPB & $0.9296 \%$ & $2.8274 \%$ & $0.7574 \%$ & $3.2237 \%$ & $2.0424 \%$ & $4.9722 \%$ & $1.7418 \%$ & $5.5510 \%$ \\
\hline SYSPEP & $64.1191 \%$ & $70.6227 \%$ & $63.0801 \%$ & $71.5825 \%$ & $55.1402 \%$ & $62.9008 \%$ & $53.9244 \%$ & $64.0635 \%$ \\
\hline SYSPM & $79.1660 \%$ & $84.5262 \%$ & $78.2722 \%$ & $85.2745 \%$ & $79.8279 \%$ & $85.8016 \%$ & $78.8199 \%$ & $86.6158 \%$ \\
\hline SYSVF & $5.9104 \%$ & $9.6532 \%$ & $5.4381 \%$ & $10.3194 \%$ & $4.1028 \%$ & $7.8810 \%$ & $3.6634 \%$ & $8.5824 \%$ \\
\hline UNKNOWN & & & & & & & & \\
\hline WPCBAPK & & & & & & & & \\
\hline WPCBASIC1 & & & & & & & & \\
\hline WPCBASIC3 & $0.0031 \%$ & $0.6727 \%$ & $0.0006 \%$ & $0.8960 \%$ & & & & \\
\hline XRAY & $31.9695 \%$ & $38.5964 \%$ & $30.9845 \%$ & $39.6485 \%$ & $30.2421 \%$ & $37.7197 \%$ & $29.1440 \%$ & $38.9121 \%$ \\
\hline XTD4C & & & & & & & & \\
\hline XTDSNT & $0.5820 \%$ & $2.2151 \%$ & $0.4514 \%$ & $2.5727 \%$ & $0.1705 \%$ & $1.5925 \%$ & $0.1052 \%$ & $1.9547 \%$ \\
\hline ZSYSBI & & & & & & & & \\
\hline
\end{tabular}


Table 24

\begin{tabular}{|c|c|c|c|c|c|c|c|c|}
\hline \multirow[t]{3}{*}{ Passed Before Shipping } & \multicolumn{4}{|c|}{ Field Failure } & \multicolumn{4}{|c|}{ Field Failure } \\
\hline & \multicolumn{4}{|c|}{ Memory Issue } & \multicolumn{4}{|c|}{ CPU Failure } \\
\hline & \multicolumn{2}{|c|}{$95 \% \mathrm{Cl}$} & \multicolumn{2}{|c|}{$99 \% \mathrm{Cl}$} & \multicolumn{2}{|c|}{$95 \% \mathrm{Cl}$} & \multicolumn{2}{|c|}{$99 \% \mathrm{Cl}$} \\
\hline Test Area & $\mathrm{LCL}$ & UCL & $\mathrm{LCL}$ & UCL & $\mathrm{LCL}$ & UCL & LCL & UCL \\
\hline ASSY & $67.6603 \%$ & $76.7542 \%$ & $66.1738 \%$ & $78.0259 \%$ & $58.2698 \%$ & $68.6631 \%$ & $56.6220 \%$ & $70.1637 \%$ \\
\hline DBGHTHV & $27.6149 \%$ & $37.1068 \%$ & $26.2595 \%$ & $38.6328 \%$ & $25.2710 \%$ & $35.1898 \%$ & $23.8783 \%$ & $36.7988 \%$ \\
\hline \multicolumn{9}{|l|}{ DBGHTLV } \\
\hline DBGLTLV & $1.7820 \%$ & $5.6184 \%$ & $1.4390 \%$ & $6.4170 \%$ & $1.8047 \%$ & $5.9798 \%$ & $1.4413 \%$ & $6.8563 \%$ \\
\hline \multicolumn{9}{|l|}{ HIPOT } \\
\hline ICT & $96.3463 \%$ & $99.2773 \%$ & $95.6745 \%$ & $99.4763 \%$ & $97.4871 \%$ & $99.8208 \%$ & $96.8637 \%$ & $99.9021 \%$ \\
\hline OSYSBI* & $0.0065 \%$ & $1.4167 \%$ & $0.0013 \%$ & $1.8847 \%$ & & & & \\
\hline PASTE & $96.3463 \%$ & 99.2773\% & $95.6745 \%$ & 99.4763\% & 97.4871\% & 99.8208\% & 96.8637\% & $99.9021 \%$ \\
\hline PCB2C & $65.7999 \%$ & $75.0616 \%$ & $64.2961 \%$ & $76.3679 \%$ & $72.9902 \%$ & $82.0191 \%$ & $71.4760 \%$ & $83.2319 \%$ \\
\hline PCBBI & $1.7820 \%$ & $5.6184 \%$ & $1.4390 \%$ & $6.4170 \%$ & $2.0155 \%$ & $6.3393 \%$ & $1.6280 \%$ & $7.2363 \%$ \\
\hline \multicolumn{9}{|c|}{ - } \\
\hline PCBBS & $0.0065 \%$ & $1.4167 \%$ & $0.0013 \%$ & $1.8847 \%$ & & & & \\
\hline PCBCEV & $0.2794 \%$ & $2.5985 \%$ & $0.1724 \%$ & $3.1858 \%$ & $1.8047 \%$ & $5.9798 \%$ & $1.4413 \%$ & $6.8563 \%$ \\
\hline PCBDG & $0.0065 \%$ & $1.4167 \%$ & $0.0013 \%$ & $1.8847 \%$ & & & & \\
\hline PCBDL & $1.7820 \%$ & $5.6184 \%$ & $1.4390 \%$ & $6.4170 \%$ & $2.4464 \%$ & $7.0496 \%$ & $2.0131 \%$ & $7.9854 \%$ \\
\hline \multicolumn{9}{|l|}{ PCBDL* } \\
\hline PCBFA & $0.5652 \%$ & $3.3099 \%$ & $0.3948 \%$ & $3.9558 \%$ & $1.1962 \%$ & $4.8802 \%$ & $0.9118 \%$ & $5.6884 \%$ \\
\hline \multicolumn{9}{|l|}{\begin{tabular}{|l|} 
PCBFA* \\
\end{tabular}} \\
\hline PCBFT & $96.0084 \%$ & $99.1126 \%$ & $95.3125 \%$ & $99.3387 \%$ & $97.4871 \%$ & $99.8208 \%$ & $96.8637 \%$ & $99.9021 \%$ \\
\hline PCBINT & $2.1628 \%$ & $6.2486 \%$ & $1.7793 \%$ & $7.0821 \%$ & $7.6418 \%$ & $14.4377 \%$ & $6.8369 \%$ & $15.6718 \%$ \\
\hline PCBP2 & $0.1585 \%$ & $2.2258 \%$ & $0.0866 \%$ & $2.7790 \%$ & $0.3159 \%$ & $2.9334 \%$ & $0.1949 \%$ & $3.5950 \%$ \\
\hline PCBPB & $2.1628 \%$ & $6.2486 \%$ & \begin{tabular}{|l|}
$1.7793 \%$ \\
\end{tabular} & $7.0821 \%$ & \begin{tabular}{|l|}
$2.6659 \%$ \\
\end{tabular} & \begin{tabular}{|l|}
$7.4011 \%$ \\
\end{tabular} & $2.2109 \%$ & $8.3551 \%$ \\
\hline PCBPB* & $0.0065 \%$ & $1.4167 \%$ & $0.0013 \%$ & $1.8847 \%$ & & & & \\
\hline PCBPEP & $0.0065 \%$ & $1.4167 \%$ & $0.0013 \%$ & $1.8847 \%$ & & & & \\
\hline PCBPM & 96.3463\% & $99.2773 \%$ & $95.6745 \%$ & $99.4763 \%$ & $96.2640 \%$ & $99.3610 \%$ & 95.5369\% & $99.5536 \%$ \\
\hline PCBST & $40.2591 \%$ & $50.3501 \%$ & $38.7483 \%$ & $51.9063 \%$ & $36.3715 \%$ & $47.0102 \%$ & $34.8048 \%$ & $48.6677 \%$ \\
\hline POSTBI & $1.5957 \%$ & $5.2996 \%$ & $1.2741 \%$ & $6.0796 \%$ & $1.5975 \%$ & $5.6172 \%$ & $1.2693 \%$ & $6.4720 \%$ \\
\hline SYSADBG & $0.0065 \%$ & $1.4167 \%$ & $0.0013 \%$ & $1.8847 \%$ & & & & \\
\hline SYSAPK & & & & & $1.8047 \%$ & $5.9798 \%$ & $1.4413 \%$ & $6.8563 \%$ \\
\hline SYSAPK* & $6.7505 \%$ & $12.8072 \%$ & $6.0364 \%$ & $13.9134 \%$ & $17.1833 \%$ & $26.0886 \%$ & $15.9944 \%$ & $27.5885 \%$ \\
\hline SYSBI & $0.1585 \%$ & $2.2258 \%$ & $0.0866 \%$ & $2.7790 \%$ & $0.4708 \%$ & $3.3400 \%$ & \begin{tabular}{|l|}
$0.3129 \%$ \\
\end{tabular} & $4.0357 \%$ \\
\hline \multicolumn{9}{|l|}{ SYSBI* } \\
\hline SYSCEV & & & & & $0.0701 \%$ & $0.0724 \%$ & $0.0300 \%$ & $2.6523 \%$ \\
\hline SYSCEV* & $0.1585 \%$ & $2.2258 \%$ & $0.0866 \%$ & $2.7790 \%$ & $1.1962 \%$ & $4.8802 \%$ & $0.9118 \%$ & $5.6884 \%$ \\
\hline SYSDL & $0.0065 \%$ & $1.4167 \%$ & $0.0013 \%$ & $1.8847 \%$ & & & & \\
\hline SYSFA & $83.2090 \%$ & $90.1319 \%$ & $81.9872 \%$ & $90.9972 \%$ & $72.0717 \%$ & $81.2188 \%$ & $70.5440 \%$ & $82.4548 \%$ \\
\hline \multicolumn{9}{|c|}{ 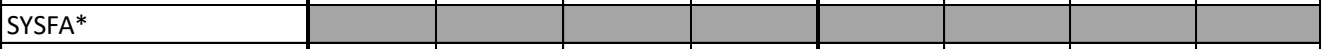 } \\
\hline SYSFT* & $85.4733 \%$ & $91.9259 \%$ & $84.3138 \%$ & $92.7086 \%$ & $73.2969 \%$ & $82.2853 \%$ & $71.7874 \%$ & $83.4902 \%$ \\
\hline SYSINT & $47.8597 \%$ & $57.9778 \%$ & $46.3079 \%$ & $59.5014 \%$ & $58.5650 \%$ & $68.9410 \%$ & $56.9182 \%$ & $70.4374 \%$ \\
\hline \multicolumn{9}{|l|}{\begin{tabular}{|l|} 
SYSINT* \\
\end{tabular}} \\
\hline SYSPB & $0.4165 \%$ & $2.9589 \%$ & $0.2767 \%$ & $3.5767 \%$ & $1.1962 \%$ & $4.8802 \%$ & $0.9118 \%$ & $5.6884 \%$ \\
\hline SYSPEP & \begin{tabular}{|l|}
$49.1677 \%$ \\
\end{tabular} & $59.2378 \%$ & $47.5826 \%$ & $60.7525 \%$ & $59.4516 \%$ & $69.7734 \%$ & $57.8085 \%$ & $71.2567 \%$ \\
\hline SYSPM & $81.2499 \%$ & $88.5395 \%$ & $79.9803 \%$ & $89.4702 \%$ & $69.9384 \%$ & $79.3414 \%$ & $68.3823 \%$ & $80.6277 \%$ \\
\hline SYSVF & $9.8681 \%$ & $16.7910 \%$ & $9.0028 \%$ & $18.0128 \%$ & $3.3382 \%$ & $8.4428 \%$ & $2.8218 \%$ & $9.4478 \%$ \\
\hline UNKNOWN & & & & & & & & \\
\hline WPCBAPK & $0.1585 \%$ & $2.2258 \%$ & $0.0866 \%$ & $2.7790 \%$ & & & & \\
\hline WPCBASIC1 & $0.1585 \%$ & $2.2258 \%$ & $0.0866 \%$ & $2.7790 \%$ & & & & \\
\hline WPCBASIC3 & & & & & & & & \\
\hline XRAY & $14.6962 \%$ & $22.6185 \%$ & $13.6503 \%$ & $23.9684 \%$ & $25.2710 \%$ & $35.1898 \%$ & $23.8783 \%$ & $36.7988 \%$ \\
\hline XTD4C & & & & & $0.0701 \%$ & $0.0724 \%$ & \begin{tabular}{|c|}
$0.0300 \%$ \\
\end{tabular} & $2.6523 \%$ \\
\hline XTDSNT & $0.0065 \%$ & $1.4167 \%$ & $0.0013 \%$ & $1.8847 \%$ & $0.0073 \%$ & $1.5997 \%$ & $0.0014 \%$ & $2.1276 \%$ \\
\hline ZSYSBI & & & & & & & & \\
\hline
\end{tabular}


Table 25

\begin{tabular}{|c|c|c|c|c|c|c|c|c|}
\hline Passed Before Shipping & & Field $\mathrm{F}$ & Failure & & & Field F & Eailure & \\
\hline & & Bootin & ig Issue & & & $\overline{D C}$ & $\overline{D A}$ & \\
\hline & $95 \%$ & $\% \mathrm{Cl}$ & $99 \%$ & $\% \mathrm{Cl}$ & $95 \%$ & $\% \mathrm{Cl}$ & $99 \%$ & $\% \mathrm{Cl}$ \\
\hline Test Area & $\mathrm{LCL}$ & UCL & $\mathrm{LCL}$ & UCL & $\mathrm{LCL}$ & $\mathrm{UCL}$ & $\mathrm{LCL}$ & UCL \\
\hline ASSY & $57.4680 \%$ & $68.6888 \%$ & $55.6914 \%$ & $70.2998 \%$ & $39.4771 \%$ & $52.1908 \%$ & $37.5976 \%$ & $54.1336 \%$ \\
\hline DBGHTHV & $36.4789 \%$ & $47.9594 \%$ & $34.7943 \%$ & $49.7424 \%$ & $29.7914 \%$ & $42.0385 \%$ & $28.0591 \%$ & $43.9852 \%$ \\
\hline DBGHTLV & & & & & & & & \\
\hline DBGLTLV & $3.6065 \%$ & $9.3476 \%$ & $3.0324 \%$ & $10.4801 \%$ & $2.8088 \%$ & $8.7624 \%$ & $2.2706 \%$ & $9.9838 \%$ \\
\hline HIPOT & & & & & & & & \\
\hline ICT & $96.6103 \%$ & 99.6343\% & $95.8481 \%$ & $99.7743 \%$ & $84.6165 \%$ & $92.7315 \%$ & $83.1127 \%$ & $93.6417 \%$ \\
\hline OSYSBI* & & & & & & & & \\
\hline PASTE & $96.6103 \%$ & $99.6343 \%$ & $95.8481 \%$ & $99.7743 \%$ & $84.6165 \%$ & $92.7315 \%$ & $83.1127 \%$ & $93.6417 \%$ \\
\hline PCB2C & $75.6577 \%$ & $84.9282 \%$ & $74.0719 \%$ & $86.1292 \%$ & $64.1856 \%$ & $75.8864 \%$ & $62.2773 \%$ & $77.4894 \%$ \\
\hline PCBBI & $3.8688 \%$ & $9.7457 \%$ & $3.2718 \%$ & $10.8965 \%$ & $3.1076 \%$ & $9.2537 \%$ & $2.5371 \%$ & $10.5007 \%$ \\
\hline PCBBI* & & & & & & & & \\
\hline PCBBS & & & & & & & & \\
\hline PCBCEV & $0.0085 \%$ & $1.8492 \%$ & $0.0017 \%$ & $2.4584 \%$ & $0.2492 \%$ & $3.4804 \%$ & $0.1361 \%$ & $4.3386 \%$ \\
\hline PCBDG & & & & & & & & \\
\hline PCBDL & $2.0907 \%$ & $6.9056 \%$ & $1.6703 \%$ & $7.9119 \%$ & $2.2256 \%$ & $7.7668 \%$ & $1.7557 \%$ & $8.9330 \%$ \\
\hline PCBDL* & & & & & & & & \\
\hline PCBFA & $0.2074 \%$ & $2.9040 \%$ & $0.1133 \%$ & $3.6227 \%$ & $0.0974 \%$ & $2.8712 \%$ & $0.0416 \%$ & $3.6704 \%$ \\
\hline PCBFA* & & & & & & & & \\
\hline PCBFT & $96.6103 \%$ & $99.6343 \%$ & $95.8481 \%$ & $99.7743 \%$ & $84.6165 \%$ & $92.7315 \%$ & $83.1127 \%$ & $93.6417 \%$ \\
\hline PCBINT & $2.0907 \%$ & $6.9056 \%$ & $1.6703 \%$ & $7.9119 \%$ & $1.1376 \%$ & $5.7062 \%$ & $0.8248 \%$ & $6.7419 \%$ \\
\hline PCBP2 & $0.0811 \%$ & $2.3953 \%$ & $0.0347 \%$ & $3.0641 \%$ & & & & \\
\hline PCBPB & $4.3999 \%$ & $10.5359 \%$ & $3.7590 \%$ & $11.7216 \%$ & $2.8088 \%$ & $8.7624 \%$ & $2.2706 \%$ & $9.9838 \%$ \\
\hline PCBPB* & & & & & & & & \\
\hline PCBPEP & & & & & $0.0102 \%$ & $2.2172 \%$ & $0.0020 \%$ & $2.9458 \%$ \\
\hline PCBPM & $96.6103 \%$ & $99.6343 \%$ & $95.8481 \%$ & $99.7743 \%$ & $84.6165 \%$ & $92.7315 \%$ & $83.1127 \%$ & $93.6417 \%$ \\
\hline PCBST & $30.0303 \%$ & $41.1643 \%$ & $28.4411 \%$ & $42.9358 \%$ & $18.5542 \%$ & $29.4733 \%$ & $17.1167 \%$ & $31.3072 \%$ \\
\hline POSTBI & $3.3466 \%$ & $8.9473 \%$ & $2.7960 \%$ & $10.0608 \%$ & $2.5147 \%$ & $8.2669 \%$ & $2.0098 \%$ & $9.4614 \%$ \\
\hline SYSADBG & & & & & & & & \\
\hline SYSAPK & $0.5451 \%$ & $3.8591 \%$ & $0.3623 \%$ & $4.6604 \%$ & & & & \\
\hline SYSAPK* & $4.1333 \%$ & $10.1417 \%$ & $3.5141 \%$ & $11.3103 \%$ & $2.2256 \%$ & $7.7668 \%$ & $1.7557 \%$ & $8.9330 \%$ \\
\hline SYSBI & & & & & & & & \\
\hline SYSBI* & & & & & & & & \\
\hline SYSCEV & $0.0085 \%$ & $1.8492 \%$ & $0.0017 \%$ & $2.4584 \%$ & & & & \\
\hline SYSCEV* & $0.0811 \%$ & $2.3953 \%$ & $0.0347 \%$ & $3.0641 \%$ & $0.0974 \%$ & $2.8712 \%$ & $0.0416 \%$ & $3.6704 \%$ \\
\hline SYSDL & & & & & & & & \\
\hline SYSFA & $87.5185 \%$ & $94.2411 \%$ & $86.2545 \%$ & $94.9825 \%$ & $77.4522 \%$ & $87.2114 \%$ & $75.7470 \%$ & $88.4223 \%$ \\
\hline SYSFA* & & & & & & & & \\
\hline SYSFT* & $88.8120 \%$ & $95.0614 \%$ & $87.4627 \%$ & $95.7440 \%$ & $79.6600 \%$ & $88.9685 \%$ & $78.0083 \%$ & $90.0951 \%$ \\
\hline SYSINT & $64.3540 \%$ & $75.0459 \%$ & $62.6169 \%$ & $76.5333 \%$ & $58.7852 \%$ & $70.9718 \%$ & $56.8418 \%$ & $72.6887 \%$ \\
\hline SYSINT* & & & & & & & & \\
\hline SYSPB & $88.8120 \%$ & $95.0614 \%$ & $87.4627 \%$ & $95.7440 \%$ & $0.2492 \%$ & $3.4804 \%$ & $0.1361 \%$ & $4.3386 \%$ \\
\hline SYSPEP & $64.3540 \%$ & $75.0459 \%$ & $62.6169 \%$ & $76.5333 \%$ & $58.3731 \%$ & $70.5904 \%$ & $56.4280 \%$ & $72.3151 \%$ \\
\hline SYSPM & $88.8120 \%$ & $95.0614 \%$ & $87.4627 \%$ & $95.7440 \%$ & $75.2670 \%$ & $85.4311 \%$ & $73.5151 \%$ & $86.7193 \%$ \\
\hline SYSVF & $64.3540 \%$ & $75.0459 \%$ & $62.6169 \%$ & $76.5333 \%$ & $5.2964 \%$ & $12.6029 \%$ & $4.5280 \%$ & $14.0028 \%$ \\
\hline UNKNOWN & & & & & & & & \\
\hline WPCBAPK & & & & & & & & \\
\hline WPCBASIC1 & & & & & & & & \\
\hline WPCBASIC3 & & & & & & & & \\
\hline XRAY & $24.9541 \%$ & $35.6460 \%$ & $23.4667 \%$ & $37.3831 \%$ & $23.3650 \%$ & $34.9761 \%$ & $21.7819 \%$ & $36.8767 \%$ \\
\hline XTD4C & & & & & & & & \\
\hline XTDSNT & $0.3657 \%$ & $3.3897 \%$ & $0.2257 \%$ & $4.1519 \%$ & $0.6551 \%$ & $4.6236 \%$ & $0.4355 \%$ & $5.5790 \%$ \\
\hline ZSYSBI & & & & & & & & \\
\hline
\end{tabular}


Table 26

\begin{tabular}{|c|c|c|c|c|c|c|c|c|}
\hline \multirow[t]{3}{*}{ Passed After Shipping } & \multicolumn{4}{|c|}{ Field Failure } & \multicolumn{4}{|c|}{ Field Failure } \\
\hline & \multicolumn{4}{|c|}{ Motherboard Issue } & \multicolumn{4}{|c|}{ Motherboard Failure } \\
\hline & \multicolumn{2}{|c|}{$95 \% \mathrm{Cl}$} & \multicolumn{2}{|c|}{$99 \% \mathrm{Cl}$} & \multicolumn{2}{|c|}{$95 \% \mathrm{Cl}$} & \multicolumn{2}{|c|}{$99 \% \mathrm{Cl}$} \\
\hline Mfg Test & $\mathrm{LCL}$ & $\mathrm{UCL}$ & $\mathrm{LCL}$ & $\mathrm{UCL}$ & $\mathrm{LCL}$ & $\mathrm{UCL}$ & $\mathrm{LCL}$ & $\mathrm{UCL}$ \\
\hline FDBGHTHV & $42.1952 \%$ & $45.1157 \%$ & $41.7428 \%$ & $45.5734 \%$ & $36.4959 \%$ & $39.8209 \%$ & $35.9854 \%$ & $40.3448 \%$ \\
\hline FPCB2C & $39.2533 \%$ & $42.1464 \%$ & $38.8064 \%$ & $42.6010 \%$ & $33.4115 \%$ & $36.6777 \%$ & $32.9119 \%$ & $37.1941 \%$ \\
\hline FPCBAPK & $38.7672 \%$ & $41.6547 \%$ & $38.3214 \%$ & $42.1087 \%$ & $33.0857 \%$ & $36.3448 \%$ & $32.5874 \%$ & $36.8602 \%$ \\
\hline FPCBDG & $0.0006 \%$ & $0.1238 \%$ & $0.0001 \%$ & $0.1651 \%$ & & & & \\
\hline \multicolumn{9}{|l|}{ FPCBFA } \\
\hline FPCBFT & $40.5577 \%$ & $43.4643 \%$ & $40.1081 \%$ & $43.9205 \%$ & $34.3894 \%$ & $37.6758 \%$ & $33.8861 \%$ & $38.1949 \%$ \\
\hline \multicolumn{9}{|l|}{ FPCBINT } \\
\hline \multicolumn{9}{|l|}{ FPCBP2 } \\
\hline \multicolumn{9}{|l|}{ FPCBPEP } \\
\hline \multicolumn{9}{|l|}{ FSYSBI } \\
\hline FSYSFA & $33.4519 \%$ & $36.2587 \%$ & $33.0210 \%$ & $36.7022 \%$ & $28.4158 \%$ & $31.5534 \%$ & $27.9392 \%$ & $32.0525 \%$ \\
\hline FSYSFT & $37.7512 \%$ & $40.6262 \%$ & $37.3078 \%$ & $41.0786 \%$ & $32.8489 \%$ & $36.1026 \%$ & $32.3515 \%$ & $36.6174 \%$ \\
\hline FSYSFT* & $1.4727 \%$ & $2.2829 \%$ & $1.3688 \%$ & $2.4273 \%$ & $0.4854 \%$ & $1.1040 \%$ & $0.4200 \%$ & $1.2257 \%$ \\
\hline FSYSINT & $33.6941 \%$ & $36.5054 \%$ & $33.2624 \%$ & $36.9495 \%$ & $28.5633 \%$ & $31.7053 \%$ & $28.0859 \%$ & $32.2050 \%$ \\
\hline FSYSPEP & $33.6721 \%$ & $36.4830 \%$ & $33.2405 \%$ & $36.9270 \%$ & $28.5633 \%$ & $31.7053 \%$ & $28.0859 \%$ & $32.2050 \%$ \\
\hline FUNKNOWN & $0.0006 \%$ & $0.1238 \%$ & $0.0001 \%$ & $0.1651 \%$ & & & & \\
\hline
\end{tabular}

Table 27

\begin{tabular}{|l|r|r|r|r|r|r|r|r|}
\hline & \multicolumn{3}{|c|}{ Field Failure } & \multicolumn{3}{c|}{ Field Failure } \\
\hline & \multicolumn{3}{|c|}{ DIMM Issue } & \multicolumn{3}{c|}{ Blade Failure } \\
\hline & \multicolumn{2}{|c|}{$95 \% \mathrm{Cl}$} & \multicolumn{2}{c|}{$99 \% \mathrm{Cl}$} & \multicolumn{2}{c|}{$95 \% \mathrm{Cl}$} & \multicolumn{2}{c|}{$99 \% \mathrm{Cl}$} \\
\hline Mfg Test & $\mathrm{LCL}$ & $\mathrm{UCL}$ & $\mathrm{LCL}$ & $\mathrm{UCL}$ & $\mathrm{LCL}$ & $\mathrm{UCL}$ & $\mathrm{LCL}$ & $\mathrm{UCL}$ \\
\hline FDBGHTHV & $28.6114 \%$ & $32.0182 \%$ & $28.0951 \%$ & $32.5603 \%$ & $44.8124 \%$ & $49.6856 \%$ & $44.0607 \%$ & $50.4435 \%$ \\
\hline FPCB2C & $27.5088 \%$ & $30.8791 \%$ & $26.9990 \%$ & $31.4162 \%$ & $42.4634 \%$ & $47.3186 \%$ & $41.7172 \%$ & $48.0764 \%$ \\
\hline FPCBAPK & $32.6873 \%$ & $36.2086 \%$ & $32.1504 \%$ & $36.7660 \%$ & $42.0423 \%$ & $46.8932 \%$ & $41.2973 \%$ & $47.6508 \%$ \\
\hline FPCBDG & $0.0009 \%$ & $0.1952 \%$ & $0.0002 \%$ & $0.2602 \%$ & $0.0015 \%$ & $0.3370 \%$ & $0.0003 \%$ & $0.4492 \%$ \\
\hline FPCBFA & & & & & $0.0147 \%$ & $0.4369 \%$ & $0.0063 \%$ & $0.5605 \%$ \\
\hline FPCBFT & $25.8572 \%$ & $29.1681 \%$ & $25.3578 \%$ & $29.6971 \%$ & $43.3662 \%$ & $48.2297 \%$ & $42.6177 \%$ & $48.9877 \%$ \\
\hline FPCBINT & & & & & $0.0147 \%$ & $0.4369 \%$ & $0.0063 \%$ & $0.5605 \%$ \\
\hline FPCBP2 & & & & & $0.0147 \%$ & $0.4369 \%$ & $0.0063 \%$ & $0.5605 \%$ \\
\hline FPCBPEP & & & & & $0.0147 \%$ & $0.4369 \%$ & $0.0063 \%$ & $0.5605 \%$ \\
\hline FSYSBI & & & & & $0.0147 \%$ & $0.4369 \%$ & $0.0063 \%$ & $0.5605 \%$ \\
\hline FSYSFA & $28.9562 \%$ & $32.3739 \%$ & $28.4380 \%$ & $32.9175 \%$ & $36.7047 \%$ & $41.4687 \%$ & $35.9794 \%$ & $42.2188 \%$ \\
\hline FSYSFT & $32.2376 \%$ & $35.7477 \%$ & $31.7026 \%$ & $36.3036 \%$ & $40.7202 \%$ & $45.5550 \%$ & $39.9793 \%$ & $46.3116 \%$ \\
\hline FSYSFT & $0.9446 \%$ & $1.8243 \%$ & $0.8434 \%$ & $1.9904 \%$ & $1.8394 \%$ & $3.4232 \%$ & $1.6535 \%$ & $3.7184 \%$ \\
\hline FSYSINT & $29.0252 \%$ & $32.4450 \%$ & $28.5066 \%$ & $32.9890 \%$ & $36.9440 \%$ & $41.7131 \%$ & $36.2176 \%$ & $42.4638 \%$ \\
\hline FSYSPEP & $29.0252 \%$ & $32.4450 \%$ & $28.5066 \%$ & $32.9890 \%$ & $36.9440 \%$ & $41.7131 \%$ & $36.2176 \%$ & $42.4638 \%$ \\
\hline FUNKNOWN & & & & & & & & \\
\hline
\end{tabular}


Table 28

\begin{tabular}{|c|c|c|c|c|c|c|c|c|}
\hline \multirow[t]{3}{*}{ Passed After Shipping } & \multicolumn{4}{|c|}{ Field Failure } & \multicolumn{4}{|c|}{ Field Failure } \\
\hline & \multicolumn{4}{|c|}{ Server Issue } & \multicolumn{4}{|c|}{ Hardware Failure } \\
\hline & \multicolumn{2}{|c|}{$95 \% \mathrm{Cl}$} & \multicolumn{2}{|c|}{$99 \% \mathrm{Cl}$} & \multicolumn{2}{|c|}{$95 \% \mathrm{Cl}$} & \multicolumn{2}{|c|}{$99 \% \mathrm{Cl}$} \\
\hline Mfg Test & LCL & $\mathrm{UCL}$ & $\mathrm{LCL}$ & $\mathrm{UCL}$ & $\mathrm{LCL}$ & $\mathrm{UCL}$ & $\mathrm{LCL}$ & $\mathrm{UCL}$ \\
\hline FDBGHTHV & $50.4053 \%$ & $57.3151 \%$ & $49.3338 \%$ & $58.3691 \%$ & $43.5722 \%$ & $51.4509 \%$ & $42.3719 \%$ & $52.6659 \%$ \\
\hline FPCB2C & $35.1660 \%$ & $41.9140 \%$ & $34.1546 \%$ & $42.9776 \%$ & $40.7898 \%$ & $48.6344 \%$ & $39.6030 \%$ & $49.8524 \%$ \\
\hline FPCBAPK & $35.1660 \%$ & $41.9140 \%$ & $34.1546 \%$ & $42.9776 \%$ & $40.3272 \%$ & $48.1638 \%$ & $39.1431 \%$ & $49.3819 \%$ \\
\hline FPCBDG & $35.9973 \%$ & $42.7715 \%$ & $34.9799 \%$ & $43.8372 \%$ & $0.0040 \%$ & $0.8675 \%$ & $0.0008 \%$ & $1.1551 \%$ \\
\hline FPCBFA & $42.4449 \%$ & $49.3521 \%$ & $41.3919 \%$ & $50.4238 \%$ & & & & \\
\hline FPCBFT & & & & & $41.5614 \%$ & $49.4180 \%$ & $40.3705 \%$ & $50.6355 \%$ \\
\hline \multicolumn{9}{|l|}{ FPCBINT } \\
\hline \multicolumn{9}{|l|}{ FPCBP2 } \\
\hline \multicolumn{9}{|l|}{ FPCBPEP } \\
\hline \multicolumn{9}{|l|}{ FSYSBI } \\
\hline FSYSFA & & & & & $38.0198 \%$ & $45.8055 \%$ & $36.8504 \%$ & $47.0225 \%$ \\
\hline FSYSFT & $46.1731 \%$ & $53.1031 \%$ & $45.1080 \%$ & $54.1699 \%$ & $39.2493 \%$ & $47.0644 \%$ & $38.0717 \%$ & $48.2823 \%$ \\
\hline FSYSFT* & $1.2965 \%$ & $3.4222 \%$ & $1.0881 \%$ & $3.8520 \%$ & $1.3176 \%$ & $3.8362 \%$ & $1.0831 \%$ & $4.3554 \%$ \\
\hline FSYSINT & $42.4449 \%$ & $49.3521 \%$ & $41.3919 \%$ & $50.4238 \%$ & $38.1733 \%$ & $45.9630 \%$ & $37.0029 \%$ & $47.1802 \%$ \\
\hline FSYSPEP & $42.4449 \%$ & $49.3521 \%$ & $41.3919 \%$ & $50.4238 \%$ & $38.1733 \%$ & $45.9630 \%$ & $37.0029 \%$ & $47.1802 \%$ \\
\hline FUNKNOWN & & & & & & & & \\
\hline
\end{tabular}

Table 29

\begin{tabular}{|c|c|c|c|c|c|c|c|c|}
\hline \multirow[t]{3}{*}{ Passed After Shipping } & \multicolumn{4}{|c|}{ Field Failure } & \multicolumn{4}{|c|}{ Field Failure } \\
\hline & \multicolumn{4}{|c|}{ Memory Issue } & \multicolumn{4}{|c|}{ CPU Failure } \\
\hline & \multicolumn{2}{|c|}{$95 \% \mathrm{Cl}$} & \multicolumn{2}{|c|}{$99 \% \mathrm{Cl}$} & \multicolumn{2}{|c|}{$95 \% \mathrm{Cl}$} & \multicolumn{2}{|c|}{$99 \% \mathrm{Cl}$} \\
\hline Mfg Test & $\mathrm{LCL}$ & $\mathrm{UCL}$ & $\mathrm{LCL}$ & $\mathrm{UCL}$ & $\mathrm{LCL}$ & UCL & $\mathrm{LCL}$ & $\mathrm{UCL}$ \\
\hline FDBGHTHV & $36.2534 \%$ & $46.2341 \%$ & $34.7794 \%$ & $47.7928 \%$ & $53.8656 \%$ & $64.4718 \%$ & $52.2084 \%$ & $66.0288 \%$ \\
\hline FPCB2C & $32.7769 \%$ & $42.6042 \%$ & $31.3438 \%$ & $44.1564 \%$ & $51.2431 \%$ & $61.9369 \%$ & $49.5864 \%$ & $63.5216 \%$ \\
\hline FPCBAPK & $33.0243 \%$ & $42.8644 \%$ & $31.5880 \%$ & $44.4173 \%$ & $50.6623 \%$ & $61.3716 \%$ & $49.0064 \%$ & $62.9618 \%$ \\
\hline \multicolumn{9}{|l|}{ FPCBDG } \\
\hline \multicolumn{9}{|l|}{ FPCBFA } \\
\hline FPCBFT & $34.7602 \%$ & $44.6818 \%$ & $33.3026 \%$ & $46.2387 \%$ & $51.8246 \%$ & $62.5015 \%$ & $50.1674 \%$ & $64.0804 \%$ \\
\hline \multicolumn{9}{|l|}{ FPCBINT } \\
\hline \multicolumn{9}{|l|}{ FPCBP2 } \\
\hline \multicolumn{9}{|l|}{ FPCBPEP } \\
\hline \multicolumn{9}{|l|}{ FSYSBI } \\
\hline FSYSFA & $28.5930 \%$ & $38.1591 \%$ & $27.2212 \%$ & $39.6916 \%$ & $43.7479 \%$ & $54.5330 \%$ & $42.1178 \%$ & $56.1725 \%$ \\
\hline FSYSFT & $31.2954 \%$ & $41.0402 \%$ & $29.8824 \%$ & $42.5869 \%$ & $49.2135 \%$ & $59.9553 \%$ & $47.5603 \%$ & $61.5584 \%$ \\
\hline FSYSFT* & $1.5957 \%$ & $5.2996 \%$ & $1.2741 \%$ & $6.0796 \%$ & $0.6390 \%$ & $3.7360 \%$ & $0.4464 \%$ & $4.4631 \%$ \\
\hline FSYSINT & $28.5930 \%$ & $38.1591 \%$ & $27.2212 \%$ & $39.6916 \%$ & $44.3202 \%$ & $55.1067 \%$ & $42.6868 \%$ & $56.7433 \%$ \\
\hline FSYSPEP & $28.5930 \%$ & $38.1591 \%$ & $27.2212 \%$ & $39.6916 \%$ & $44.3202 \%$ & $55.1067 \%$ & $42.6868 \%$ & $56.7433 \%$ \\
\hline FUNKNOWN & & & & & & & & \\
\hline
\end{tabular}


Table 30

\begin{tabular}{|c|c|c|c|c|c|c|c|c|}
\hline \multirow[t]{3}{*}{ Passed After Shipping } & \multicolumn{4}{|c|}{ Field Failure } & \multicolumn{4}{|c|}{ Field Failure } \\
\hline & \multicolumn{4}{|c|}{ Booting Issue } & \multicolumn{4}{|c|}{$\mathrm{DOA}$} \\
\hline & \multicolumn{2}{|c|}{$95 \% \mathrm{Cl}$} & \multicolumn{2}{|c|}{$99 \% \mathrm{Cl}$} & \multicolumn{2}{|c|}{$95 \% \mathrm{Cl}$} & \multicolumn{2}{|c|}{$99 \% \mathrm{Cl}$} \\
\hline Mfg Test & LCL & $\mathrm{UCL}$ & $\mathrm{LCL}$ & $\mathrm{UCL}$ & $\mathrm{LCL}$ & $\mathrm{UCL}$ & $\mathrm{LCL}$ & $\mathrm{UCL}$ \\
\hline FDBGHTHV & $45.6824 \%$ & $57.2975 \%$ & $43.9184 \%$ & $59.0427 \%$ & $38.3011 \%$ & $50.9861 \%$ & $36.4351 \%$ & $52.9336 \%$ \\
\hline FPCB2C & $43.6935 \%$ & $55.3132 \%$ & $41.9414 \%$ & $57.0716 \%$ & $37.1288 \%$ & $49.7779 \%$ & $35.2774 \%$ & $51.7289 \%$ \\
\hline FPCBAPK & $42.7025 \%$ & $54.3176 \%$ & $40.9573 \%$ & $56.0816 \%$ & $36.7388 \%$ & $49.3743 \%$ & $34.8925 \%$ & $51.3262 \%$ \\
\hline \multicolumn{9}{|l|}{ FPCBDG } \\
\hline \multicolumn{9}{|l|}{ FPCBFA } \\
\hline FPCBFT & $43.6935 \%$ & $55.3132 \%$ & $41.9414 \%$ & $57.0716 \%$ & $37.5191 \%$ & $50.1810 \%$ & $35.6628 \%$ & $52.1310 \%$ \\
\hline \multicolumn{9}{|l|}{ FPCBINT } \\
\hline \multicolumn{9}{|l|}{ FPCBP2 } \\
\hline \multicolumn{9}{|l|}{ FPCВРЕР } \\
\hline \multicolumn{9}{|l|}{ FSYSBI } \\
\hline FSYSFA & $38.4342 \%$ & $49.9772 \%$ & $36.7275 \%$ & $51.7573 \%$ & $33.6340 \%$ & $46.1309 \%$ & $31.8332 \%$ & $48.0857 \%$ \\
\hline FSYSFT & $42.3726 \%$ & $53.9852 \%$ & $40.6300 \%$ & $55.7510 \%$ & $35.1831 \%$ & $47.7559 \%$ & $33.3585 \%$ & $49.7103 \%$ \\
\hline FSYSFT* & $0.7399 \%$ & $4.3163 \%$ & $0.5170 \%$ & $5.1533 \%$ & $1.1376 \%$ & $5.7062 \%$ & $0.8248 \%$ & $6.7419 \%$ \\
\hline FSYSINT & $38.4342 \%$ & $49.9772 \%$ & $36.7275 \%$ & $51.7573 \%$ & $33.6340 \%$ & $46.1309 \%$ & $31.8332 \%$ & $48.0857 \%$ \\
\hline FSYSPEP & $38.4342 \%$ & $49.9772 \%$ & $36.7275 \%$ & $51.7573 \%$ & $33.6340 \%$ & $46.1309 \%$ & $31.8332 \%$ & $48.0857 \%$ \\
\hline FUNKNOWN & & & & & & & & \\
\hline
\end{tabular}

The results from Tables 1-30 were used to identify the critical test areas for the top 10 field failures, which are shown in Tables 31-40. Any test area that has a confidence interval that contains $10 \%$ or above was chosen for failed and repaired tests and $40 \%$ or above was chosen for passed tests. If the tests in these areas were run first recognizing the problems of the product would be achieved faster and repairs for those problems could be scheduled earlier, even while the rest of the testing was still being performed. Also, with a ranking of the proportion of the population that will be failed, repaired, or passed each test on Tables 41-52, if the product continues to have problems after the critical tests are performed, the product can be sent to the next test area in the ranking knowing that there is more of a chance to find the problem in one area over another. The distinguishing of these critical tests saves time and labor and would increase the amount of useful information collected while decreasing the amount of unproductive information. 
Table 31

Critical Test Areas- Most Critical to Least Critical Over $10 \%$

Failed Before Shipping Field Failure C

\begin{tabular}{|l|l|l|}
\hline & \multicolumn{1}{|c|}{$95 \% \mathrm{Cl}$} & \multicolumn{1}{c|}{$99 \% \mathrm{Cl}$} \\
\hline Test Areas & SYSFA & SYSFA \\
\hline
\end{tabular}

Over $10 \%$

Failed After Shipping

Field Failure

\begin{tabular}{|l|l|l|} 
& \multicolumn{2}{|c|}{ Motherboard Issue } \\
\hline Test Areas & FPCBFT & \multicolumn{1}{c|}{$99 \% \mathrm{CI}$} \\
\hline & FDBGHTHV & FPCBFT \\
\hline
\end{tabular}

Over 10\%

Repaired Before Shipping Field Failure

\begin{tabular}{|c|c|c|}
\hline & & \\
\hline & $95 \% \mathrm{Cl}$ & $99 \% \mathrm{Cl}$ \\
\hline Test & IPAOI & IPAOI \\
\hline
\end{tabular}

Over 10\%

Repaired After Shipping Field Failure

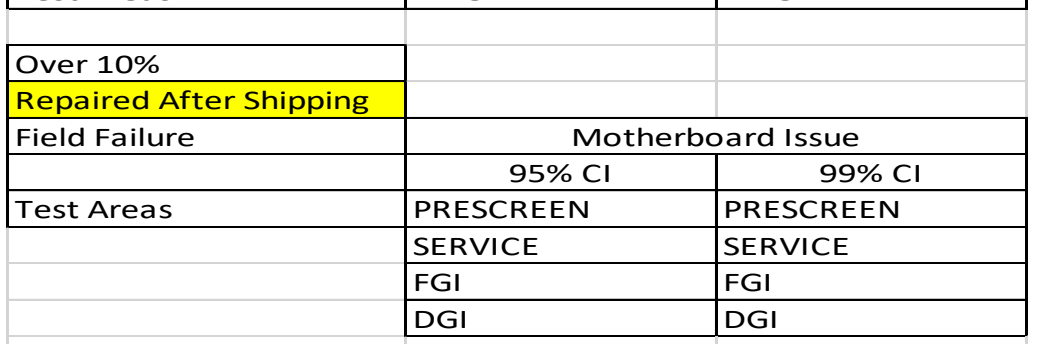

\begin{tabular}{|l|}
\hline Over $40 \%$ \\
\hline Passed Before Shipping \\
\hline Field Failure \\
\hline
\end{tabular}

Field Failure

Test Areas

\begin{tabular}{|l|l|}
\multicolumn{2}{|c|}{ Motherboard Issue } \\
\hline \multicolumn{2}{|c|}{$95 \% \mathrm{CI}$} \\
\hline ICT & \multicolumn{1}{|c|}{$99 \% \mathrm{CI}$} \\
\hline PCBFT & ICT \\
\hline PASTE & PCBFT \\
\hline PCBPM & PASTE \\
\hline SYSFT* & PCBPM \\
\hline SYSFA & SYSFT* \\
\hline SYSPM & SYSFA \\
\hline PCB2C & SYSPM \\
\hline SYSPEP & PCB2C \\
\hline ASSY & SYSPEP \\
\hline SYSINT & ASSY \\
\hline PCBST & SYSINT \\
\hline
\end{tabular}

Over $40 \%$

Passed After Shipping

Field Failure

\begin{tabular}{|l|l|l|}
\hline \multicolumn{1}{|c|}{$95 \% \mathrm{CI}$} & \multicolumn{1}{c|}{$99 \% \mathrm{Cl}$} \\
\hline Test Areas & \multicolumn{1}{|c|}{ FDBGHTHV } & FDBGHTHV \\
\hline \multirow{5}{*}{} & FPCBFT & FPCBFT \\
\hline & FPCB2C & FPCB2C \\
\hline & FPCBAPK & FPCBAPK \\
\hline & FSYSFT & FSYSFT \\
\cline { 2 - 3 }
\end{tabular}


Table 32

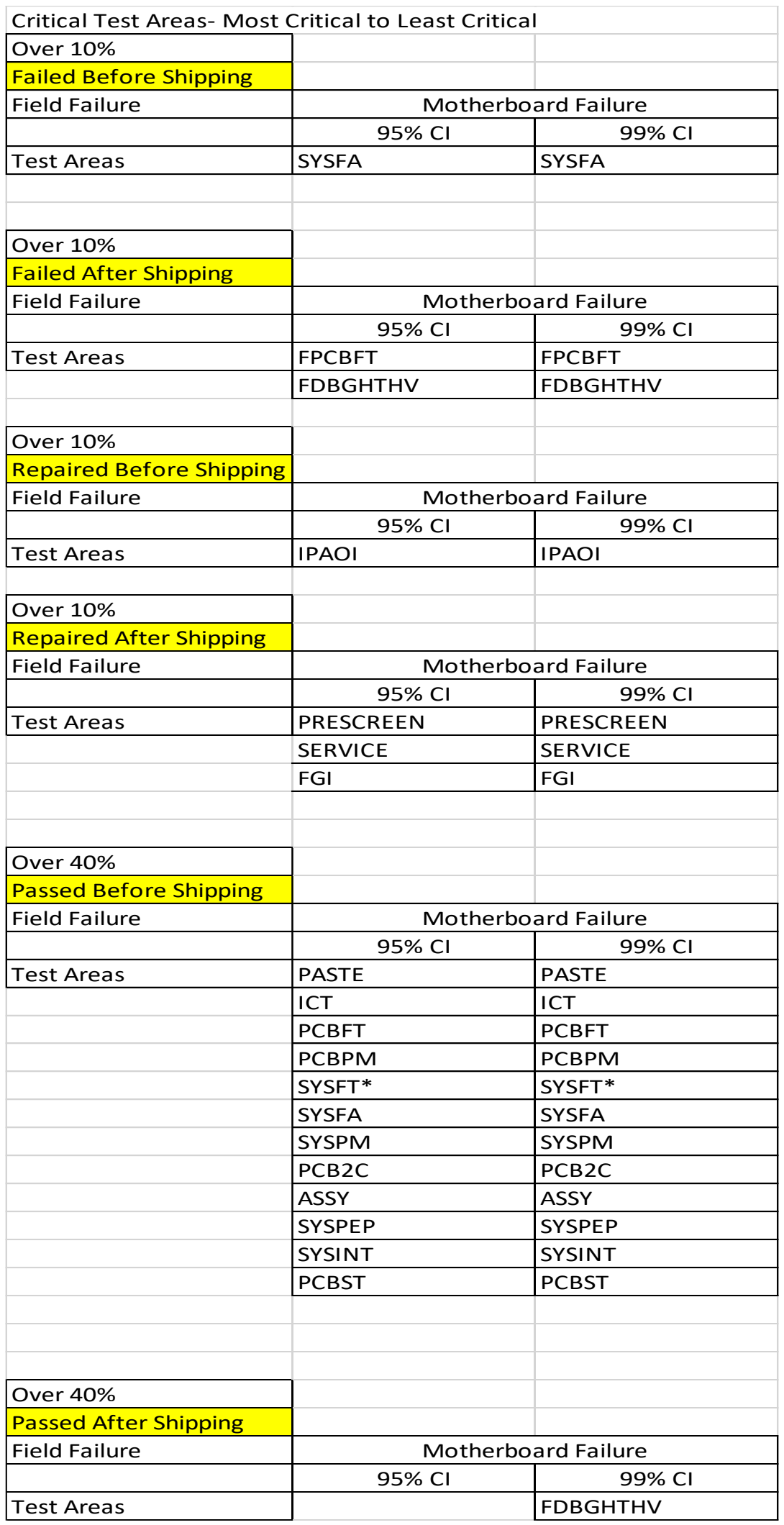


Table 33

Critical Test Areas- Most Critical to Least Critical Over 10\%

Failed Before Shipping Field Failure

\begin{tabular}{|l|c|c|}
\hline & \multicolumn{2}{|c|}{ DIMM Issue } \\
\hline Test Areas & $95 \% \mathrm{Cl}$ & $99 \% \mathrm{CI}$ \\
\hline
\end{tabular}

\begin{tabular}{|c|c|c|}
\hline Over 10\% & & \\
\hline \multicolumn{3}{|l|}{ Failed After Shipping } \\
\hline \multirow[t]{2}{*}{ Field Failure } & \multicolumn{2}{|c|}{ DIMM Issue } \\
\hline & $95 \% \mathrm{Cl}$ & $99 \% \mathrm{Cl}$ \\
\hline \multicolumn{3}{|l|}{\begin{tabular}{|l|} 
Test Areas \\
\end{tabular}} \\
\hline \multicolumn{3}{|l|}{ Over $10 \%$} \\
\hline \multicolumn{3}{|l|}{ Repaired Before Shipping } \\
\hline \multirow[t]{2}{*}{ Field Failure } & \multicolumn{2}{|c|}{ DIMM Issue } \\
\hline & $95 \% \mathrm{Cl}$ & $99 \% \mathrm{Cl}$ \\
\hline Test Areas & IPAOI & IPAOI \\
\hline
\end{tabular}

\begin{tabular}{|l|l|l|}
\hline Over $10 \%$ & \multicolumn{2}{|c|}{} \\
\cline { 1 - 1 } Repaired After Shipping & \multicolumn{2}{|c|}{} \\
\hline Field Failure & \multicolumn{2}{|c|}{ DIMM Issue } \\
\hline & $95 \% \mathrm{Cl}$ & \multicolumn{1}{|c|}{$99 \% \mathrm{CI}$} \\
\hline Test Areas & PRESCREEN & PRESCREEN \\
\hline & FGI & FGI \\
\hline & SERVICE & SERVICE \\
\hline & & \\
\hline
\end{tabular}

\begin{tabular}{|c|c|c|}
\hline Over $40 \%$ & & \\
\hline \multicolumn{3}{|l|}{ Passed Before Shipping } \\
\hline \multirow[t]{2}{*}{ Field Failure } & \multicolumn{2}{|c|}{ DIMM Issue } \\
\hline & $95 \% \mathrm{Cl}$ & $99 \% \mathrm{Cl}$ \\
\hline \multirow[t]{12}{*}{ Test Areas } & PASTE & PASTE \\
\hline & PCBPM & PCBPM \\
\hline & ICT & ICT \\
\hline & PCBFT & PCBFT \\
\hline & SYSFT* & SYSFT* \\
\hline & SYSPM & SYSPM \\
\hline & ASSY & ASSY \\
\hline & SYSFA & SYSFA \\
\hline & PCB2C & PCB2C \\
\hline & SYSPEP & SYSPEP \\
\hline & SYSINT & SYSINT \\
\hline & PCBST & PCBST \\
\hline
\end{tabular}

\begin{tabular}{|c|c|c|}
\hline \multicolumn{3}{|l|}{ Over 40\% } \\
\hline \multicolumn{3}{|l|}{ Passed After Shipping } \\
\hline \multirow[t]{2}{*}{ Field Failure } & \multicolumn{2}{|c|}{ DIMM Issue } \\
\hline & $95 \% \mathrm{Cl}$ & $99 \% \mathrm{Cl}$ \\
\hline Test Areas & & \\
\hline
\end{tabular}


Table 34

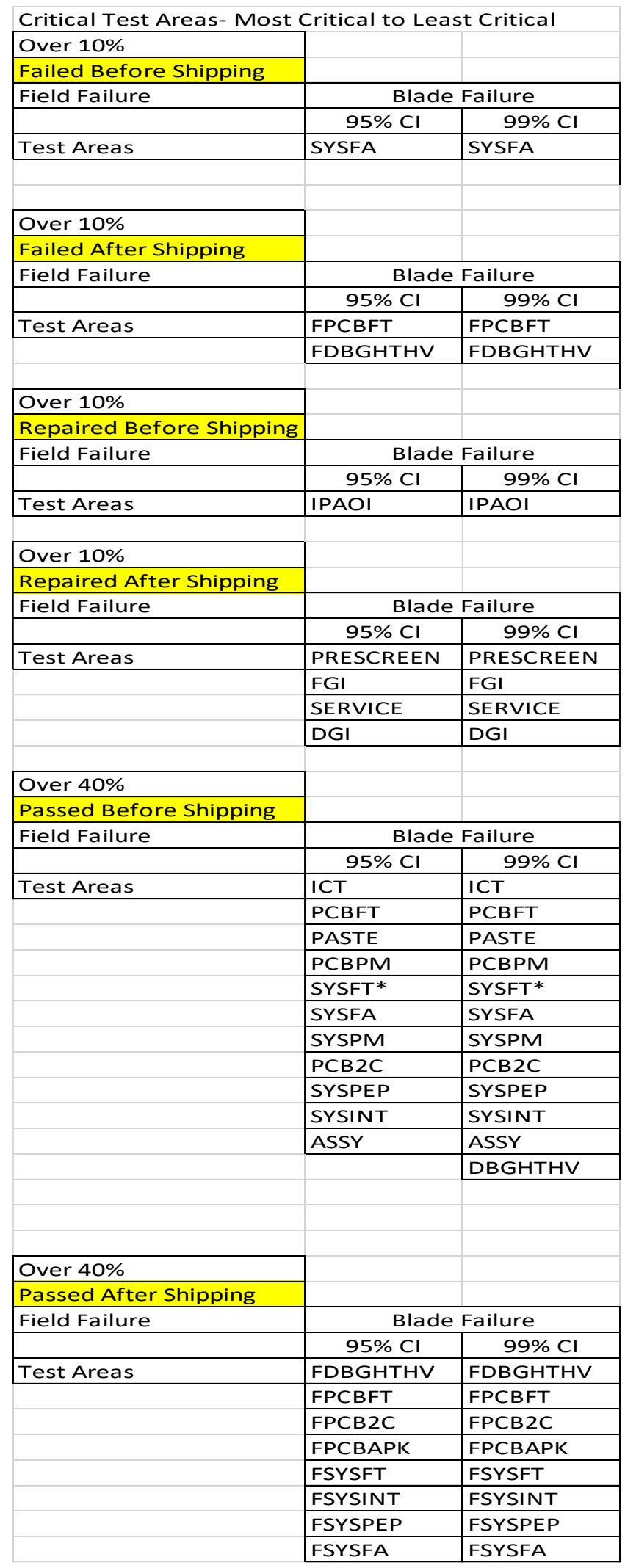


Table 35

Critical Test Areas- Most Critical to Least Critical Over 10\%

Failed Before Shipping

Field Failure

\begin{tabular}{|c|c|c|}
\hline \multirow[t]{2}{*}{ Field Failure } & \multicolumn{2}{|c|}{ Server Issue } \\
\hline & $95 \% \mathrm{Cl}$ & $99 \% \mathrm{Cl}$ \\
\hline \multirow[t]{2}{*}{ Test Areas } & SYSFA & SYSFA \\
\hline & PCBFT & PCBFT \\
\hline \multicolumn{3}{|l|}{ Over $10 \%$} \\
\hline \multicolumn{3}{|c|}{ Failed After Shipping } \\
\hline \multirow[t]{2}{*}{ Field Failure } & \multicolumn{2}{|c|}{ Server Issue } \\
\hline & $95 \% \mathrm{Cl}$ & $99 \% \mathrm{Cl}$ \\
\hline \multirow[t]{3}{*}{ Test Areas } & FPCBFT & FPCBFT \\
\hline & FDBGHTHV & FDBGHTHV \\
\hline & FPCB2C & FPCB2C \\
\hline \multicolumn{3}{|l|}{ Over $10 \%$} \\
\hline \multicolumn{3}{|c|}{ Repaired Before Shipping } \\
\hline \multirow[t]{2}{*}{ Field Failure } & \multicolumn{2}{|c|}{ Server Issue } \\
\hline & $95 \% \mathrm{Cl}$ & $99 \% \mathrm{Cl}$ \\
\hline Test Areas & IPAOI & IPAOI \\
\hline
\end{tabular}

\begin{tabular}{|c|c|c|}
\hline Over 10\% & & \\
\hline \multicolumn{3}{|l|}{ Repaired After Shipping } \\
\hline \multirow{2}{*}{\begin{tabular}{|l|} 
Field Failure \\
\end{tabular}} & \multicolumn{2}{|c|}{ Server Issue } \\
\hline & $95 \% \mathrm{Cl}$ & $99 \% \mathrm{Cl}$ \\
\hline \multirow[t]{4}{*}{\begin{tabular}{|l|} 
Test Areas \\
\end{tabular}} & PRESCREEN & PRESCREEN \\
\hline & FGI & FGI \\
\hline & SERVICE & SERVICE \\
\hline & DGI & DGI \\
\hline
\end{tabular}

\begin{tabular}{|c|c|c|}
\hline Over $40 \%$ & & \\
\hline Passed Before Shipping & & \\
\hline Field Failure & \multicolumn{2}{|c|}{ Server Issue } \\
\hline & $95 \% \mathrm{Cl}$ & $99 \% \mathrm{Cl}$ \\
\hline \multirow[t]{13}{*}{ Test Areas } & ICT & ICT \\
\hline & PCBFT & PCBFT \\
\hline & PASTE & PASTE \\
\hline & PCBPM & PCBPM \\
\hline & SYSFT* & SYSFT* \\
\hline & SYSFA & SYSFA \\
\hline & SYSPM & SYSPM \\
\hline & PCB2C & РСB2C \\
\hline & SYSPEP & SYSPEP \\
\hline & SYSINT & SYSINT \\
\hline & ASSY & ASSY \\
\hline & DBGHTHV & DBGHTHV \\
\hline & & PCBST \\
\hline \multicolumn{3}{|l|}{ Over $40 \%$} \\
\hline \multicolumn{3}{|l|}{ Passed After Shipping } \\
\hline \multirow[t]{2}{*}{ Field Failure } & \multicolumn{2}{|c|}{ Server Issue } \\
\hline & $95 \% \mathrm{Cl}$ & $99 \% \mathrm{Cl}$ \\
\hline \multirow[t]{8}{*}{ Test Areas } & FDBGHTHV & FDBGHTHV \\
\hline & FSYSFT & FSYSFT \\
\hline & FSYSINT & FSYSINT \\
\hline & FSYSPEP & FSYSPEP \\
\hline & FPCBFA & FPCBFA \\
\hline & FPCBDG & FPCBDG \\
\hline & FPCB2C & FPCB2C \\
\hline & FPCBAPK & FPCBAPK \\
\hline
\end{tabular}


Table 36

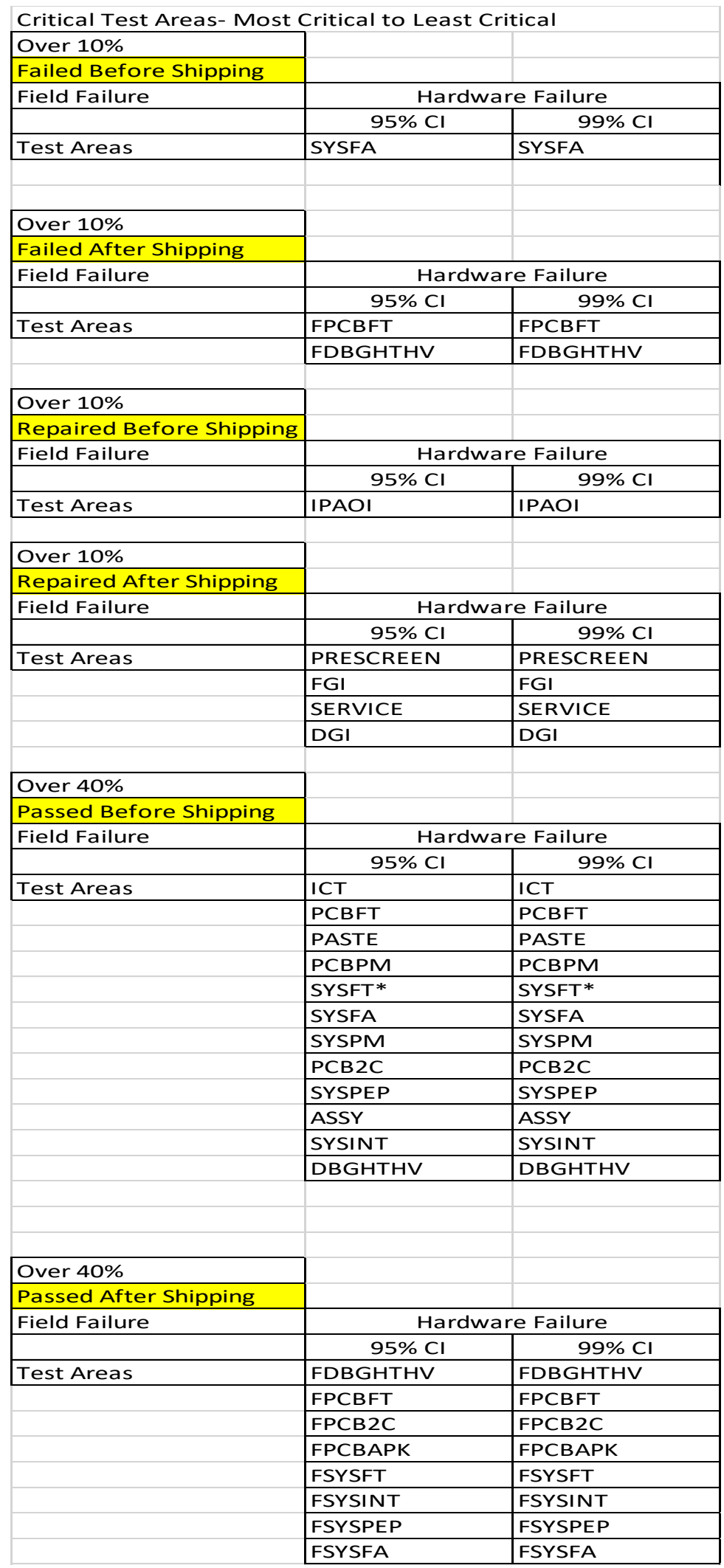


Table 37

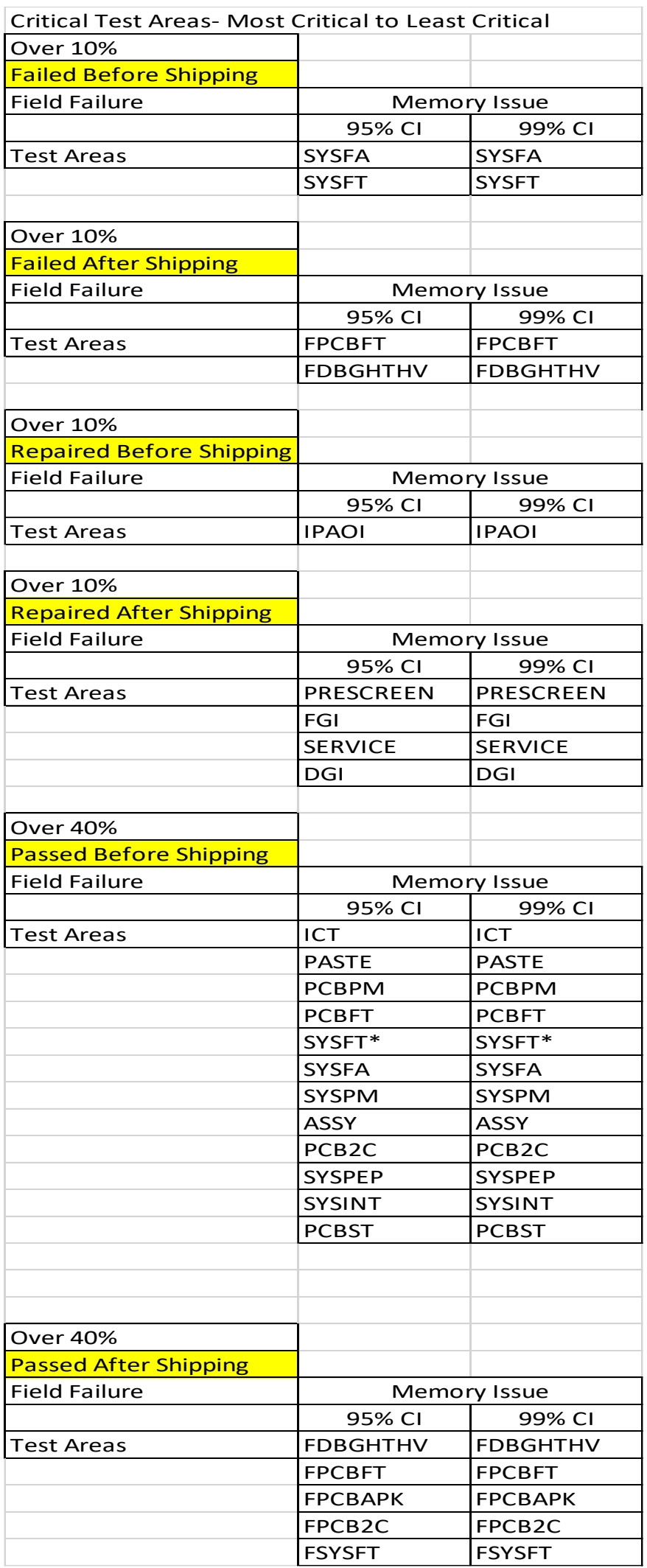


Table 38

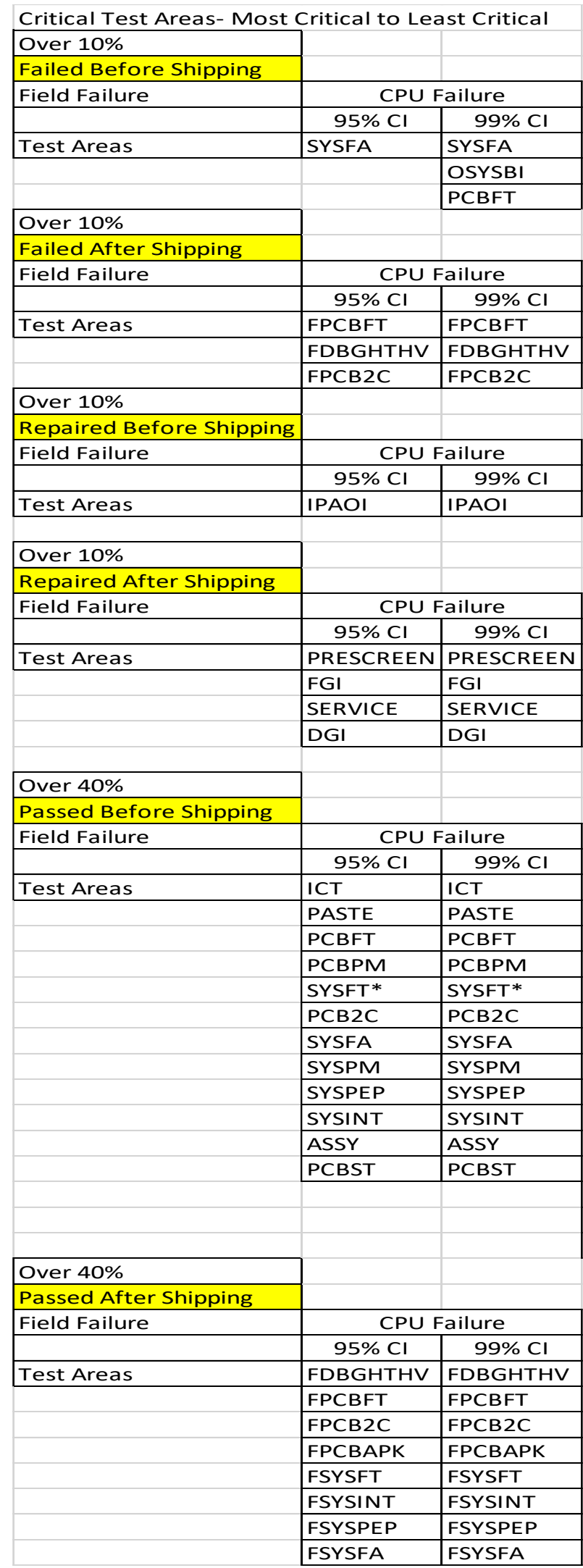


Table 39

Critical Test Areas- Most Critical to Least Critical Over 10\%

Failed Before Shipping Field Failure

\begin{tabular}{|c|c|}
\hline \multicolumn{2}{|c|}{ Booting Issue } \\
\hline $95 \% \mathrm{Cl}$ & $99 \% \mathrm{CI}$ \\
\hline SYSFA & SYSFA \\
\hline PCBFT & PCBFT \\
\hline & SYSFT \\
\hline
\end{tabular}

\begin{tabular}{|c|c|c|}
\hline \multicolumn{3}{|l|}{ Over $10 \%$} \\
\hline \multicolumn{3}{|l|}{ Failed After Shipping } \\
\hline \multirow[t]{2}{*}{ Field Failure } & \multicolumn{2}{|c|}{ Booting Issue } \\
\hline & $95 \% \mathrm{Cl}$ & $99 \% \mathrm{Cl}$ \\
\hline \multirow[t]{3}{*}{ Test Areas } & FPCBFT & FPCBFT \\
\hline & FDBGHTHV & FDBGHTHV \\
\hline & FPCB2C & FPCB2C \\
\hline \multicolumn{3}{|l|}{ Over $10 \%$} \\
\hline \multicolumn{3}{|l|}{ Repaired Before Shipping } \\
\hline \multirow[t]{2}{*}{ Field Failure } & \multicolumn{2}{|c|}{ Booting Issue } \\
\hline & $95 \% \mathrm{Cl}$ & $99 \% \mathrm{Cl}$ \\
\hline Test Areas & IPAOI & IPAOI \\
\hline
\end{tabular}

\begin{tabular}{|c|c|c|}
\hline Over $10 \%$ & & \\
\hline \multicolumn{3}{|l|}{ Repaired After Shipping } \\
\hline \multirow[t]{2}{*}{ Field Failure } & \multicolumn{2}{|c|}{ Booting Issue } \\
\hline & $95 \% \mathrm{Cl}$ & $99 \% \mathrm{Cl}$ \\
\hline \multirow[t]{4}{*}{ Test Areas } & PRESCREEN & PRESCREEN \\
\hline & SERVICE & SERVICE \\
\hline & FGI & FGI \\
\hline & DGI & DGI \\
\hline
\end{tabular}

\begin{tabular}{|c|c|c|}
\hline Over $40 \%$ & & \\
\hline Passed Before Shipping & & \\
\hline Field Failure & \multicolumn{2}{|c|}{ Booting Issue } \\
\hline & $95 \% \mathrm{Cl}$ & $99 \% \mathrm{Cl}$ \\
\hline \multirow[t]{15}{*}{ Test Areas } & ICT & ICT \\
\hline & PASTE & PASTE \\
\hline & PCBFT & PCBFT \\
\hline & PCBPM & PCBPM \\
\hline & SYSFT* & SYSFT* \\
\hline & SYSPM & SYSPM \\
\hline & SYSPB & SYSPB \\
\hline & SYSFA & SYSFA \\
\hline & PCB2C & PCB2C \\
\hline & SYSPEP & SYSPEP \\
\hline & SYSINT & SYSINT \\
\hline & SYSVF & SYSVF \\
\hline & ASSY & ASSY \\
\hline & DBGHTHV & DBGHTHV \\
\hline & PCBST & PCBST \\
\hline \multicolumn{3}{|l|}{ Over $40 \%$} \\
\hline \multicolumn{3}{|l|}{ Passed After Shipping } \\
\hline \multirow[t]{2}{*}{ Field Failure } & \multicolumn{2}{|c|}{ Booting Issue } \\
\hline & $95 \% \mathrm{Cl}$ & $99 \% \mathrm{Cl}$ \\
\hline \multirow[t]{8}{*}{ Test Areas } & FDBGHTHV & FDBGHTHV \\
\hline & FPCBFT & FPCBFT \\
\hline & FPCB2C & FPCB2C \\
\hline & FPCBAPK & FPCBAPK \\
\hline & FSYSFT & FSYSFT \\
\hline & FSYSINT & FSYSINT \\
\hline & FSYSPEP & FSYSPEP \\
\hline & FSYSFA & FSYSFA \\
\hline
\end{tabular}


Table 40

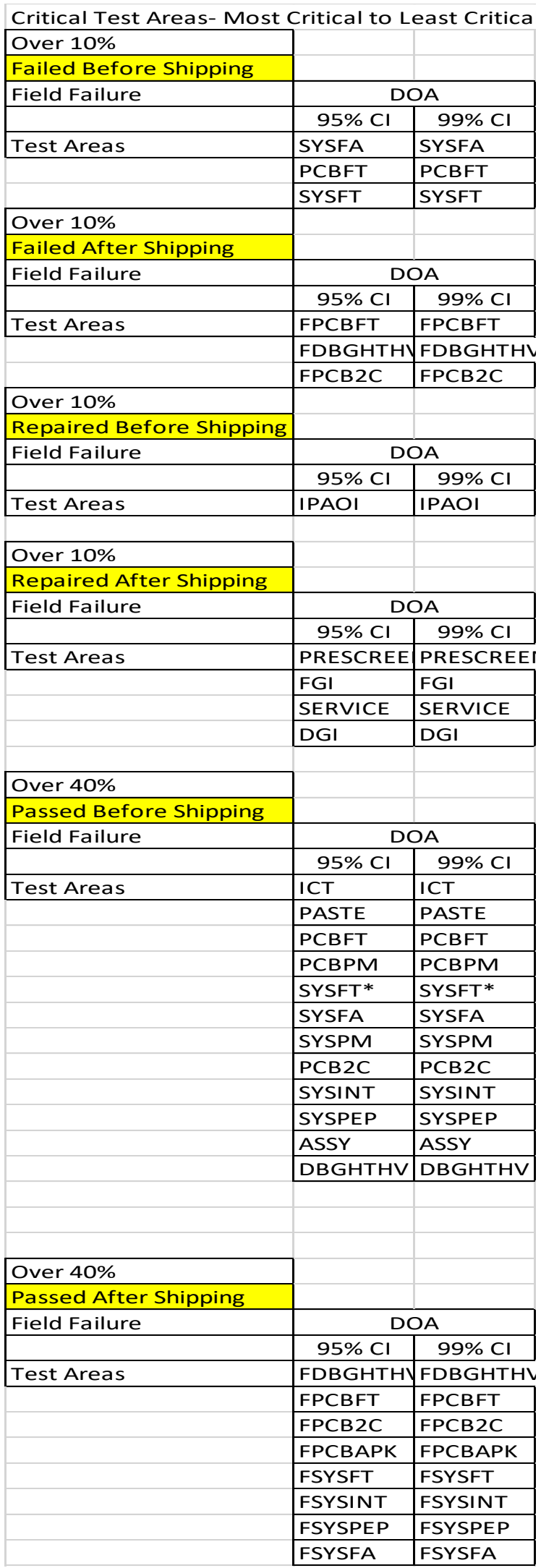


Table 41

\begin{tabular}{|c|c|c|c|c|c|}
\hline \multicolumn{6}{|l|}{ Failed Before Shipping } \\
\hline Field Failure & Motherboard Issue & Motherboard Failure & DIMM Issue & Blade Failure & Server Issue \\
\hline \multirow[t]{25}{*}{ Test Areas } & SYSFA & SYSFA & SYSFA & SYSFA & SYSFA \\
\hline & PCBFT & PCBFT & SYSFT & PCBFT & PCBFT \\
\hline & SYSFT & SYSFT & PCBFT & PCB2C & SYSFT \\
\hline & PCB2C & PCBST & PCB2C & SYSFT & PCB2C \\
\hline & PCBST & PCB2C & PCBST & ICT & ICT \\
\hline & ICT & ICT & ICT & PCBST & DBGHTHV \\
\hline & DBGHTHV & DBGHTHV & DBGHTHV & DBGHTHV & PCBST \\
\hline & \begin{tabular}{|l|} 
PCBDL \\
\end{tabular} & SYSAPK & XRAY & PCBDL & SYSAPK \\
\hline & SYSAPK & PCBDL & SYSAPK & SYSPB & PCBDL \\
\hline & XRAY & XRAY & PCBDL & XRAY & SYSINT \\
\hline & PCBBI & XTDSNT & POSTBI & SYSINT & PCBBI \\
\hline & SYSINT & PCBBI & XTDSNT & PCBBI & POSTBI \\
\hline & XTDSNT & SYSINT & PCBBI & SYSBI & XRAY \\
\hline & \begin{tabular}{|l|} 
POSTBI \\
\end{tabular} & POSTBI & SYSBI & SYSAPK & XTDSNT \\
\hline & PCBFA & SYSPM & SYSPB & PCBFA & SYSPB \\
\hline & SYSPM & PCBFA & OSYSBI & POSTBI & SYSPM \\
\hline & RSYSBI & SYSBI & SYSINT & SYSADBG & PCBFA \\
\hline & SYSBI & PCBP2 & SYSPM & XTDSNT & PCBP2 \\
\hline & OSYSBI & SYSCEV & ОРСВРВ & PCBP2 & PCBPEP \\
\hline & PCBPEP & PCBCEV & PCBFA & RSYSBI & SYSADBG \\
\hline & SYSPB & SYSPB & SYSCEV & SYSPM & \\
\hline & PCBP2 & SYSPEP & SYSPEP & UNKNOWN & \\
\hline & SYSADBG & XTD4C & & & \\
\hline & SYSPEP & & & & \\
\hline & UNKNOWN & & & & \\
\hline
\end{tabular}


Table 42

\begin{tabular}{|c|c|c|c|c|c|}
\hline Failed Before Shipping & & & & & \\
\hline Field Failure & Hardware Failure & Memory Issue & CPU Failure & Booting Issue & $\mathrm{DOA}$ \\
\hline \multirow[t]{19}{*}{ Test Areas } & SYSFA & SYSFA & SYSFA & SYSFA & SYSFA \\
\hline & SYSFT & SYSFT & OSYSBI & PCBFT & PCBFT \\
\hline & PCBFT & PCBFT & PCBFT & SYSFT & SYSFT \\
\hline & PCB2C & PCBST & SYSFT & PCBST & DBGHTHV \\
\hline & PCBST & PCB2C & PCBST & ICT & PCB2C \\
\hline & ICT & ICT & ICT & PCB2C & ICT \\
\hline & DBGHTHV & DBGHTHV & DBGHTHV & DBGHTHV & PCBST \\
\hline & PCBDL & PCBDL & PCB2C & POSTBI & XRAY \\
\hline & PCBFA & SYSPB & PCBDL & SYSAPK & PCBBI \\
\hline & SYSINT & XRAY & SYSPB & XRAY & POSTBI \\
\hline & XRAY & PCBBI & XRAY & PCBDL & SYSBI \\
\hline & OSYSBI & RSYSBI & POSTBI & SYSBI & WPCBASIC1 \\
\hline & PCBBI & SYSBI & SYSAPK & SYSINT & \\
\hline & POSTBI & WPCBASIC1 & SYSBI & SYSPB & \\
\hline & SYSADBG & PCBFA & SYSPM & XTDSNT & \\
\hline & SYSAPK & POSTBI & XTDSNT & & \\
\hline & SYSPB & SYSADBG & & & \\
\hline & SYSPM & & & & \\
\hline & XTDSNT & & & & \\
\hline
\end{tabular}

Table 43

\begin{tabular}{|l|l|l|l|l|}
\hline Failed After Shipping & \multicolumn{5}{|l|}{} & \\
\hline Field Failure & Motherboard Issue & Motherboard Failure & DIMM Issue & Blade Failure \\
\hline Test Areas & FPCBFT & FPCBFT & FPCBFT & FPCBFT \\
\hline & FDBGHTHV & FDBGHTHV & FDBGHTHV & FDBGHTHV \\
\hline & FPCB2C & FPCB2C & FPCB2C & FPCB2C \\
\hline & FPCBDG & FPCBDG & FPCBDG & FPCBDG \\
\hline & FPCBAPK & FPCBAPK & FPCBAPK & FPCBAPK \\
\hline & FSYSFT & FSYSFT & FSYSFT & FSYSFT \\
\hline & & & & FUNKNOWN \\
\hline
\end{tabular}


Table 44

\begin{tabular}{|l|l|l|l|l|l|}
\hline Failed After Shipping & \multicolumn{2}{|l|}{} \\
\hline Field Failure & Hardware Failure & Memory Issue & CPU Failure & Booting Issue & DOA \\
\hline Test Areas & FPCBFT & FPCBFT & FPCBFT & FPCBFT & FPCBFT \\
\hline & FDBGHTHV & FDBGHTHV & FDBGHTHV & FDBGHTHV & FDBGHTHV \\
\hline & FPCB2C & FPCB2C & FPCB2C & FPCB2C & FPCB2C \\
\hline & FPCBDG & FPCBDG & FPCBDG & FPCBDG & FPCBDG \\
\hline & FPCBAPK & FPCBAPK & & FPCBAPK & FSYSFT \\
\hline & FSYSFT & & & FPCBAPK \\
\hline & FPCBP2 & & & & \\
\hline
\end{tabular}

Table 45

\begin{tabular}{|l|l|l|l|l|l|}
\hline Repaired Before Shipping & & & \\
\hline Field Failure & Motherboard Issue & Motherboard Failure & DIMM Issue & Blade Failure & Server Issue \\
\hline Test Areas & IPAOI & IPAOI & IPAOI & IPAOI \\
\hline & IPAOI & IPMVI & IPMVI & IPMVI & IPMVI \\
\hline & IPMVI & SYSFA & SYSFA & SYSFA & SYSFA \\
\hline & SYSFA & SYSFT & PCBDG & SYSFT & SYSFT \\
\hline & SYSFT & DBGHTHV & DBGHTHV & DBGHTHV & DBGHTHV \\
\hline & PCBDG & PCBDG & SYSFT & PCBFA & PCBDG \\
\hline & DBGHTHV & SYSPB & SYSPB & PCB2C & ICT \\
\hline & PCBFT & PCBFA & & \\
\hline & SYSBI & & & & \\
\hline & SYSPB & & & \\
\hline
\end{tabular}

Table 46

\begin{tabular}{|l|l|l|l|l|l|}
\hline Repaired Before Shipping & \multicolumn{2}{|l|}{} \\
\hline Field Failure & Hardware Failure & Memory Issue & CPU Failure & Booting Issue & DOA \\
\hline Test Areas & IPAOI & IPAOI & IPAOI & IPAOI & IPAOI \\
\hline & IPMVI & IPMVI & IPMVI & IPMVI & IPMVI \\
\cline { 2 - 5 } & SYSFA & PCBFA & SYSFA & SYSFA & SYSFA \\
\cline { 2 - 5 } & DBGHTHV & DBGHTHV & DBGHTHV & PCBDG & PCBDG \\
\hline & SYSFT & PCBFT & SYSFT & SYSFT & PCBBI \\
\hline & SYSPB & PCBDG & PCB2C & PCBFT & \\
\hline & PCBFT & & PCBBI & & \\
\hline
\end{tabular}

Table 47

\begin{tabular}{|l|l|l|l|l|l|l|}
\hline Repaired After Shipping & \multicolumn{2}{|l|}{} \\
\hline Field Failure & Motherboard Issue & Motherboard Failure & DIMM Issue & Blade Failure & Server Issue \\
\hline Test Areas & PRESCREEN & PRESCREEN & PRESCREEN & PRESCREEN & PRESCREEN \\
\hline & SERVICE & SERVICE & FGI & FGI & FGI \\
\cline { 2 - 6 } & FGI & FGI & SERVICE & SERVICE & SERVICE \\
\cline { 2 - 6 } & DGI & DGI & DGI & DGI & DGI \\
\cline { 2 - 6 }
\end{tabular}


Table 48

\begin{tabular}{|l|l|l|l|l|l|}
\hline Repaired After Shipping & & & & \\
\hline Field Failure & Hardware Failure & Memory Issue & CPU Failure & Booting Issue & DOA \\
\hline Test Areas & PRESCREEN & PRESCREEN & PRESCREEN & PRESCREEN & PRESCREEN \\
\hline & FGI & FGI & FGI & SERVICE & FGI \\
\cline { 2 - 6 } & SERVICE & SERVICE & SERVICE & FGI & SERVICE \\
\cline { 2 - 6 } & DGI & DGI & DGI & DGI & DGI \\
\hline
\end{tabular}


Table 49

\begin{tabular}{|c|c|c|c|c|c|}
\hline Passed Before Shipping & & & & & \\
\hline Field Failure & Motherboard Issue & Motherboard Failure & DIMM Issue & Blade Failure & Server Issue \\
\hline \multirow[t]{40}{*}{ Test Areas } & ICT & PASTE & PASTE & ICT & ICT \\
\hline & PCBFT & ICT & PCBPM & PCBFT & PCBFT \\
\hline & PASTE & PCBFT & ICT & PASTE & PASTE \\
\hline & PCBPM & PCBPM & PCBFT & PCBPM & PCBPM \\
\hline & SYSFT* & SYSFT* & SYSFT* & SYSFT* & SYSFT* \\
\hline & SYSFA & SYSFA & SYSPM & SYSFA & SYSFA \\
\hline & SYSPM & SYSPM & ASSY & SYSPM & SYSPM \\
\hline & РСB2C & PCB2C & SYSFA & РCB2C & PCB2C \\
\hline & SYSPEP & ASSY & РCB2C & SYSPEP & SYSPEP \\
\hline & ASSY & SYSPEP & SYSPEP & SYSINT & SYSINT \\
\hline & SYSINT & SYSINT & SYSINT & ASSY & ASSY \\
\hline & PCBST & PCBST & PCBST & DBGHTHV & DBGHTHV \\
\hline & DBGHTHV & DBGHTHV & DBGHTHV & XRAY & PCBST \\
\hline & XRAY & XRAY & XRAY & PCBST & XRAY \\
\hline & SYSAPK* & SYSAPK* & SYSAPK* & SYSAPK* & SYSAPK* \\
\hline & SYSVF & PCBINT & SYSVF & РСВРВ & SYSVF \\
\hline & PCВРВ & SYSVF & PCBINT & PCBINT & PCBINT \\
\hline & PCBINT & PCBPB & РCВРВ & PCBDL & PCBPB \\
\hline & PCBBI & DBGLTLV & DBGLTLV & PCBBI & DBGLTLV \\
\hline & DBGLTLV & PCBBI & PCBBI & DBGLTLV & POSTBI \\
\hline & POSTBI & POSTBI & POSTBI & POSTBI & PCBBI \\
\hline & PCBDL & SYSAPK & PCBCEV & SYSPB & PCBDL \\
\hline & PCBFA & PCBDL & SYSAPK & PCBFA & PCBCEV \\
\hline & SYSPB & PCBCEV & PCBDL & SYSVF & SYSCEV* \\
\hline & XTDSNT & SYSCEV* & SYSCEV* & SYSBI & SYSPB \\
\hline & SYSAPK & SYSPB & SYSPB & XTDSNT & PCBFA \\
\hline & РСBP2 & PCBFA & PCBFA & PCBCEV & XTDSNT \\
\hline & PCBCEV & XTDSNT & PCBP2 & PCBP2 & SYSAPK \\
\hline & SYSBI & SYSBI & XTDSNT & SYSCEV* & SYSBI \\
\hline & SYSCEV* & SYSCEV & SYSCEV & SYSADBG & PCBP2 \\
\hline & SYSCEV & PCBP2 & SYSDL & SYSAPK & SYSADBG \\
\hline & SYSDL & SYSDL & SYSBI & SYSCEV & SYSCEV \\
\hline & SYSADBG & XTD4C & PCBDL* & SYSBI* & WPCBASIC3 \\
\hline & XTD4C & WPCBASIC3 & XTD4C & SYSDL & \\
\hline & OSYSBI* & PCBDG & WPCBASIC1 & PCBPEP & \\
\hline & PCBPB* & PCBDL* & OSYSBI* & ZSYSBI & \\
\hline & WPCBASIC3 & PCBFA* & PCBPB* & XTD4C & \\
\hline & DBGHTLV & SYSFA* & PCBBI* & WPCBASIC1 & \\
\hline & & SYSINT* & UNKNOWN & UNKNOWN & \\
\hline & & WPCBASIC1 & WPCBAPK & & \\
\hline
\end{tabular}


Table 50

\begin{tabular}{|c|c|c|c|c|c|}
\hline Passed Before Shipping & & & & & \\
\hline Field Failure & Hardware Failure & Memory Issue & CPU Failure & Booting Issue & $\mathrm{DOA}$ \\
\hline \multirow[t]{38}{*}{ Test Areas } & ICT & ICT & ICT & ICT & ICT \\
\hline & PCBFT & PASTE & PASTE & PASTE & PASTE \\
\hline & PASTE & PCBPM & PCBFT & PCBFT & PCBFT \\
\hline & PCBPM & PCBFT & PCBPM & PCBPM & PCBPM \\
\hline & SYSFT* & SYSFT* & SYSFT* & SYSFT* & SYSFT* \\
\hline & SYSFA & SYSFA & PCB2C & SYSPM & SYSFA \\
\hline & SYSPM & SYSPM & SYSFA & SYSPB & SYSPM \\
\hline & PCB2C & ASSY & SYSPM & SYSFA & PCB2C \\
\hline & SYSPEP & PCB2C & SYSPEP & PCB2C & SYSINT \\
\hline & ASSY & SYSPEP & SYSINT & SYSPEP & SYSPEP \\
\hline & SYSINT & SYSINT & ASSY & SYSINT & ASSY \\
\hline & DBGHTHV & PCBST & PCBST & SYSVF & DBGHTHV \\
\hline & XRAY & DBGHTHV & DBGHTHV & ASSY & XRAY \\
\hline & PCBST & XRAY & XRAY & DBGHTHV & PCBST \\
\hline & SYSAPK* & SYSVF & SYSAPK* & PCBST & SYSVF \\
\hline & PCBPB & SYSAPK* & PCBINT & XRAY & PCBBI \\
\hline & PCBINT & PCBPB & SYSVF & PCBPB & PCBPB \\
\hline & SYSVF & PCBINT & PCBPB & SYSAPK* & DBGLTLV \\
\hline & DBGLTLV & DBGLTLV & PCBDL & PCBBI & POSTBI \\
\hline & PCBBI & PCBBI & PCBBI & DBGLTLV & SYSAPK* \\
\hline & PCBDL & PCBDL & DBGLTLV & POSTBI & PCBDL \\
\hline & POSTBI & POSTBI & PCBCEV & PCBINT & PCBINT \\
\hline & SYSPB & PCBFA & SYSAPK & PCBDL & XTDSNT \\
\hline & SYSAPK & SYSPB & POSTBI & SYSAPK & SYSPB \\
\hline & PCBFA & PCBCEV & PCBFA & XTDSNT & PCBCEV \\
\hline & SYSBI & SYSBI & SYSPB & PCBFA & PCBFA \\
\hline & PCBCEV & SYSCEV* & SYSCEV* & SYSCEV* & SYSCEV* \\
\hline & \begin{tabular}{|l|} 
SYSCEV* \\
\end{tabular} & PCBP2 & SYSBI & PCBP2 & PCBPEP \\
\hline & PCBP2 & WPCBASIC1 & PCBP2 & PCBCEV & \\
\hline & XTDSNT & WPCBAPK & SYSCEV & SYSCEV & \\
\hline & SYSCEV & XTDSNT & XTD4C & & \\
\hline & SYSADBG & SYSADBG & \begin{tabular}{|l|} 
XTDSNT \\
\end{tabular} & & \\
\hline & SYSDL & SYSDL & & & \\
\hline & HIPOT & PCBPEP & & & \\
\hline & & OSYSBI* & & & \\
\hline & & PCBPB* & & & \\
\hline & & PCBDG & & & \\
\hline & & PCBBS & & & \\
\hline
\end{tabular}


Table 51

\begin{tabular}{|l|l|l|l|l|l|}
\hline Passed After Shipping & & & \\
\hline Field Failure & Motherboard Issue & Motherboard Failure & DIMM Issue & Blade Failure & Server Issue \\
\hline Test Areas & FDBGHTHV & FDBGHTHV & FDBGHTHV & FDBGHTHV & FDBGHTHV \\
\hline & FPCBFT & FPCBFT & FPCBFT & FPCBFT & FSYSFT \\
\hline & FPCB2C & FPCB2C & FPCB2C & FPCB2C & FSYSINT \\
\hline & FPCBAPK & FPCBAPK & FPCBAPK & FPCBAPK & FSYSPEP \\
\hline & FSYSFT & FSYSFT & FSYSFT & FSYSFT & FPCBFA \\
\hline & FSYSINT & FSYSINT & FSYSINT & FSYSINT & FPCBDG \\
\hline & FSYSPEP & FSYSPEP & FSYSPEP & FSYSPEP & FPCB2C \\
\hline & FSYSFA & FSYSFA & FSYSFA & FSYSFA & FPCBAPK \\
\hline & FSYSFT $*$ FSYSFT & FSYSFT* & FSYSFT* & FSYSFT* \\
\hline & FPCBDG & & FPCBDG & FPCBFA & \\
\hline & FUNKNOWN & & & FPCBINT & \\
\hline & & & & FPCBP2 \\
\hline & & & & FPCBPEP & \\
\hline & & & & FSYSBI & \\
\hline & & & & \\
\hline
\end{tabular}

Table 52

\begin{tabular}{|l|l|l|l|l|l|}
\hline Passed After Shipping & & & & \\
\hline Field Failure & Hardware Failure & Memory Issue & CPU Failure & Booting Issue & DOA \\
\hline Test Areas & FDBGHTHV & FDBGHTHV & FDBGHTHV & FDBGHTHV & FDBGHTHV \\
\hline & FPCBFT & FPCBFT & FPCBFT & FPCBFT & FPCBFT \\
\hline & FPCB2C & FPCBAPK & FPCB2C & FPCB2C & FPCB2C \\
\hline & FPCBAPK & FPCB2C & FPCBAPK & FPCBAPK & FPCBAPK \\
\hline & FSYSFT & FSYSFT & FSYSFT & FSYSFT & FSYSFT \\
\hline & FSYSINT & FSYSINT & FSYSINT & FSYSINT & FSYSINT \\
\hline & FSYSPEP & FSYSPEP & FSYSPEP & FSYSPEP & FSYSPEP \\
\hline & FSYSFA & FSYSFA & FSYSFA & FSYSFA & FSYSFA \\
\hline & FSYSFT & FSYSFT* & FSYSFT* & FSYSFT* & FSYSFT* \\
\hline & FPCBDG & & & & \\
\hline
\end{tabular}

\subsection{Comparison Table}

The comparison table includes each of the unique serial numbers from the RMA data set

that have at least one failed test in the CRMC data set. It lists the name of the failed test 
and a count of how many times the test was failed. The failed tests are separated by

before shipping to the customer and after return so that comparisons can be made. Table

53 shows a sample of the comparison table that was created as the whole table consists of

13,214 rows which is too large to be included in full in this report.

Table 53

\begin{tabular}{|c|c|c|c|c|c|c|}
\hline Serial Numbers & Field Failure & Manufacturing Test Failures (before shipping) & Count & Return Date & Manufacturing Test Failures (return from the field) & Count \\
\hline FCH152770M3 & Blade Failure & & & $1 / 20 / 2015$ & VERIFY_UUID & 1 \\
\hline $\mathrm{FCH} 15327174$ & Power Up Issue & COTATI_TEST_PREP & 1 & $5 / 5 / 2014$ & & \\
\hline FCH15327178 & Hardware Failure & COTATI_TEST_PREP & 1 & $1 / 15 / 2013$ & & \\
\hline \multirow[t]{3}{*}{$\mathrm{FCH} 154673 \mathrm{HJ}$} & Blade Failure & failed digital or boundary scan tes & 1 & $8 / 22 / 2013$ & & \\
\hline & & DIAG_FAIL:HOSTSYS_-_CPU-EXT & 1 & & & \\
\hline & & UEFI_TEST_FAIL:_IOHX64**PCIE_ER & 1 & & & \\
\hline \multirow[t]{7}{*}{ FCH160175GV } & Blade Failure & View File & 1 & $3 / 19 / 2013$ & & \\
\hline & & MEMORY_COUNT_MISMATCH & 4 & & & \\
\hline & & DETECT_SUBASSEMBLIES & 2 & & & \\
\hline & & TELNET_BMC & 1 & & & \\
\hline & & BIOS_UPDATE|CO & 1 & & & \\
\hline & & UEFI_TEST_FAIL:_PCIX64**VERIFY_ & 2 & & & \\
\hline & & BLADE_STATUS/DISC_FAILED & 2 & & & \\
\hline \multirow[t]{6}{*}{ FCH160278ZC } & Motherboard Failure & BUILD_TEST_CONFIG & 1 & $12 / 23 / 2016$ & & \\
\hline & & TAN_MISSING & 4 & & & \\
\hline & & PCBDL_AREACHECK & 2 & & & \\
\hline & & ENQUEUE_CMD & 1 & & & \\
\hline & & failed analog test & 2 & & & \\
\hline & & failed power supply test & 2 & & & \\
\hline \multirow[t]{17}{*}{ FCH16027907 } & Motherboard Issue & failed analog test & 2 & $12 / 10 / 2014$ & COTATI_FW_CHK & 1 \\
\hline & & SEL_UNCORR_ERR $\mid C O$ & 2 & & PMEM_TEST $\mid C 1$ & 1 \\
\hline & & CMOS_BAT_CHK $\mid 2.024 \mathrm{~V}$ & 1 & & EFI_DSH_TEST|C1 & 1 \\
\hline & & SEL_UNCORR_ERR $\mid C 1$ & 1 & & & \\
\hline & & AREA_CHECK & 2 & & & \\
\hline & & VERIFY_DIAG_VERSION & 2 & & & \\
\hline & & DIAG_VERSION & 3 & & & \\
\hline & & VERIFY_BMC_VERSION|CO & 1 & & & \\
\hline & & INVOKE_DIAGS & 2 & & & \\
\hline & & UEFI_TEST_FAIL:_ICHX64**VERIFY_ & 1 & & & \\
\hline & & LSI_CHECKS & 1 & & & \\
\hline & & PCB2C_AREACHECK & 1 & & & \\
\hline & & COTATI_TEST_PREP & 1 & & & \\
\hline & & CD_TO_FS1/FSO(VMEDIA):|CO & 1 & & & \\
\hline & & UCSB-B200-M3_CNF_NOT_FOUND & 1 & & & \\
\hline & & BLADE_DISCOVERY & 6 & & & \\
\hline & & UPDATE_ADAPTER & 1 & & & \\
\hline
\end{tabular}

The full comparison chart will be given to the testers at Cisco to allow them to look at the failed tests before and after the product is sent to the customer and see what failed tests from before may be associated with failed tests after shipping. This will allow them to test after shipping for specific cases based on what tests failed before shipping which will save time because unnecessary tests will not be run. 


\section{CONCLUSIONS}

Cisco wanted to know how field failure categories relate to manufacturing test failures to better predict the tests that should be performed on a product after a return by a customer and what tests will be failed by a product before and after a return by a customer based on what type of failure occurred in the field. The data was filtered and sorted by field failure, shipping status, test area result, and test area. For each combination of field failure, shipping status, test area result, and test area a statistical model of the population proportion was created. The $95 \%$ and $99 \%$ confidence intervals were found. The critical test areas are listed in Tables 31-40 and a ranking of the criticality of the test areas are listed in Tables 41-52. These findings will reduce the time spent on unnecessary tests. 


\section{RECOMMENDATIONS FOR FUTURE WORK}

This study looked at only one product type, UCSB-B200-M3, and in the future the methods in this study should be expanded to all other product types at Cisco. Also, different types of statistical analysis should be tried such as hypothesis testing for population proportion to approximate the actual population proportion with statistical

significance. In the future, the method in this study and the ones mentioned above should be automated so that they can be run monthly or daily to track changes in failure patterns and rates. 


\section{WORKS CITED}

Biswas, Sanjib, and Jaydip Sen. "A Proposed Architecture for Big Data Driven Supply Chain Analytics." IUP Journal of Supply Chain Management 13.3 (2016): 7-33. ProQuest. Web. 2 June 2017.

Brennan, Louis, et al. "Manufacturing in the World: Where Next?" International Journal of Operations \& Production Management 35.9 (2015): 1253-74. ProQuest. Web. 31 May 2017.

Russom, Philip. "Big Data Analytics." TDWI best practices report, fourth quarter 19 (2011): 40. 1 June 2017.

Wang, Lidong, and Cheryl Ann Alexander. "Big Data Driven Supply Chain Management and Business Administration." American Journal of Economics and Business Administration. Science Publications, 20 June 2015. Web. 31 May 2017. <http://thescipub.com/abstract/10.3844/ajebasp.2015.60.67>. 